\title{
Resource Management in Wireless Cellular Networks with Heterogeneous Capacity Supply and Heterogeneous Traffic Demand (HetHetNets)
}

by

\section{Ziyang Wang}

A thesis submitted to the

Faculty of Graduate and Postdoctoral Affairs

in partial fulfillment of the requirements for the degree of

Master of Applied Science in Electrical and Computer Engineering

Ottawa-Carleton Institute for Electrical and Computer Engineering

Department of Systems and Computer Engineering

Carleton University

Ottawa, Ontario

September, 2014

(c) Copyright

Ziyang Wang, 2014 
The undersigned hereby recommends to the

Faculty of Graduate and Postdoctoral Affairs acceptance of the thesis

\title{
Resource Management in Wireless Cellular Networks with Heterogeneous Capacity Supply and Heterogeneous Traffic Demand (HetHetNets)
}

\author{
submitted by Ziyang Wang \\ in partial fulfillment of the requirements for the degree of \\ Master of Applied Science in Electrical and Computer Engineering \\ Professor Halim Yanikomeroglu, Thesis Co-Supervisor \\ Professor Marc St-Hilaire, Thesis Co-supervisor \\ Professor Nancy Samaan, Internal Examiner \\ Professor Roshdy Hafez, Chair \\ Department of Systems and Computer Engineering \\ Ottawa-Carleton Institute for Electrical and Computer Engineering \\ Department of Systems and Computer Engineering \\ Carleton University \\ September, 2014
}




\section{Abstract}

The term HetNets (heterogeneous networks) is used to describe cellular networks in which the capacity supply is heterogeneous due to the overlaid architecture of macrocells and small-cells.

In this thesis we use the term HetHetNets to refer to cellular networks in which the traffic demand is also heterogeneous due to reasons such as user spatial clustering and diversified user traffic types, in addition to the heterogeneity in the capacity supply.

The main objective of this thesis is to study the essential problem of matching the traffic demand ("load") with the capacity supply ("capacity") in resource-limited HetHetNets; this problem is expected to be a major concern in the fifth generation $(5 \mathrm{G})$ cellular networks.

This thesis proposes two approaches to address this problem. One approach is to deploy small-cells to the centers of user clusters (put supply where needed), and another approach is to guide users to the cells that are lightly loaded (load balancing via spatial traffic shaping).

We first investigate how to model the spatial non-uniform (i.e., heterogeneous) user distribution, and then the impact of user spatial heterogeneity on HetNets is evaluated. Simulation-based analyses show that network performance deteriorates significantly with the increase in user spatial heterogeneity if the user locations are uncorrelated with the locations of the macro and small-cell base stations. Cluster analysis on the non-uniform user points is then utilized to find the cluster centroids as the potential locations for small-cells. Simulation results show that the network performance can improve substantially with increasing user spatial heterogeneity if we deploy small-cells in the appropriate locations.

The second approach is enabled by the recently developed user-in-the-loop (UIL) paradigm. In the literature, there have been several investigations on load-aware cell association as an approach to match traffic demand with the capacity supply, in which a user may associate to a less loaded cell, even though that cell does not 
necessarily provide the maximum SINR. In other words, a user is associated with a cell to get more share of resources at the cost of lower spectral efficiency. While, the UIL approach can increase the user received SINR and the share of allocated resources at the same time, which encourages a user to move to a new location that maximizes the utility function considering received SINR, cell load and the probability of moving. Numerical results show that the UIL can double the mean user rate in comparison to the load-aware cell association strategy, and also results in a more balanced load across the network. 
I would like to dedicate my thesis to my wife Wuyi and my son Dale. 


\section{Acknowledgments}

First and foremost, I am extremely grateful to my supervisors Prof. Halim Yanikomeroglu and Prof. Marc St-Hilaire for their guidance, support, generosity, motivation and encouragement during the past two years. Without Prof. Marc StHilaire's recognition, I would not have had the chance to come to Carleton University, and without Prof. Halim Yanikomeroglu's appreciation, I would not have had the chance to be a member of a prestigious research group that I have benefited so much from.

I am also extremely thankful to Dr. Rainer Schoenen for his consistent guidance and encouragement. He has spent so much effort on my research, and his broad knowledge has saved me from losing directions so many times.

Sincere thanks to all my colleagues for their support in both my research and my personal life, especially to Mehmet Cagri Ilter, Hamza Umit Sokun, Yaser Fouad, Meisam Mirahan, Irem Bor and Ramy Gohary. This brotherhood has made me stronger and optimistic when I am under difficulties.

I would also like to thank my friend and mentor Qiangmin $\mathrm{Yu}$ for giving me enlightenment that has changed my life.

On the personal side, I want to thank my family for their unconditional love. Without their support and faith in me, I would not be the man I am today. 


\section{Table of Contents}

Abstract $\quad$ iii

Acknowledgments $\quad$ vi

Table of Contents vii

List of Tables $\quad$ x

List of Figures $\quad$ xi

List of Abbreviations xiii

1 Introduction $\quad 1$

1.1 Definition ........................... 1

1.2 Problem Statement and Motivation . . . . . . . . . . . . 1

1.3 Contributions and Organization . . . . . . . . . . . . 3

2 Model for User Spatial Heterogeneity 6

2.1 Point Processes in Stochastic Geometry . . . . . . . . . . . . . . . . . 6

2.1.1 Poisson Point Process . . . . . . . . . . . . . . . . 7

2.1.2 Binomial Point Process . . . . . . . . . . . . . . 7

2.1.3 Hard Core Point Process . . . . . . . . . . . . . . . 8

2.1.4 Poisson Cluster Process . . . . . . . . . . . . 8

2.2 Log Gaussian Cox Process . . . . . . . . . . . . . . . . 8

2.3 Measure of User Spatial Heterogeneity . . . . . . . . . . . . . . . . . 12

2.3.1 Related Work . . . . . . . . . . . . . . . . . . . 13

2.3.2 Unified and Nonparameterized Metrics . . . . . . . . . . . . 14

2.4 Summary . . . . . . . . . . . . . . . . 16 
3 The Impact of User Spatial Heterogeneity 17

3.1 Guidelines of Performance Evaluation . . . . . . . . . . . . . . . . 17

3.1 .1 Network Layout . . . . . . . . . . . . . . . . . . . . . . . . . 18

3.1.2 Antenna Characteristics . . . . . . . . . . . . . . 19

3.1.3 Channel Model . . . . . . . . . . . . . . . . . 21

3.2 Finite User Rate Demand and Resource Allocation Scheme . . . . . . 23

3.3 Performance Evaluation . . . . . . . . . . . . . . . 27

3.3.1 The Impact of User Spatial Heterogeneity . . . . . . . . . . . 27

3.3.2 The Impact of Finite Rate Demand . . . . . . . . . . . . . . . 29

3.4 Summary . . . . . . . . . . . . . . . . . . 30

4 Pushing Capacity Supply to Traffic Demand 31

4.1 Related Work . . . . . . . . . . . . . . . . . . . . . . . . . 31

4.2 Cluster Analysis . . . . . . . . . . . . . . . . . . . 32

4.2.1 Basic k-means Algorithm . . . . . . . . . . . . . . . . 32

4.2.2 Preprocessing and Postprocessing . . . . . . . . . . . . . 33

4.2.3 Selection Criteria . . . . . . . . . . . . . . . . . . . . 34

4.3 Performance Evaluation . . . . . . . . . . . . . . . . . . 37

4.3.1 Small-cell Deployment Strategy . . . . . . . . . . . . . . 37

4.3 .2 Fairness Index . . . . . . . . . . . . . . . . . . . 39

4.4 Summary . . . . . . . . . . . . . . . . . . . . 41

5 Pushing Traffic Demand to Capacity Supply 42

5.1 Related Work . . . . . . . . . . . . . . . . . . . 43

5.2 System Model for Spatial Traffic Shaping . . . . . . . . . . . . . . . . 44

5.3 User Model . . . . . . . . . . . . . . . . . . . . . . . 46

5.3.1 User Traffic Classes . . . . . . . . . . . . . . . . . . . . 46

5.3.2 Resource Allocation . . . . . . . . . . . . . . . . . . 47

5.3.3 Probability of Moving: the P function . . . . . . . . . . . . . 48

5.4 Proposed UIL Load Balancing Approach . . . . . . . . . . . . . 50

5.4.1 Utility Function of GBR Users . . . . . . . . . . . . . . . . 51

5.4 .2 Utility Function of BE Users . . . . . . . . . . . . . . . . . 51

5.4.3 Sequential Optimization . . . . . . . . . . . . . 52

5.4.4 An Example of Load Balancing Achieved by UIL . . . . . . . 53

5.4.5 Outlook: Include Mobility Model . . . . . . . . . . . . . . 54 
5.5 Load-Aware Cell Association . . . . . . . . . . . . . . . . . . . 54

5.5 .1 Related Work . . . . . . . . . . . . . . . . . . 56

5.5 .2 Proposed Scheme . . . . . . . . . . . . . . . . 57

$5.6 \quad$ Numerical Results . . . . . . . . . . . . . . . . . . . . . . . . . 58

5.6 .1 Simulation Setup . . . . . . . . . . . . . . . . . 58

5.6.2 Transient and Steady State Behavior . . . . . . . . . . 60

5.6 .3 Loads among Different Cells . . . . . . . . . . . . . . . 61

5.6 .4 GBR Users Outage . . . . . . . . . . . . . . . . . . 63

5.6 .5 Mean User Rate . . . . . . . . . . . . . . . . . . . . . . . 65

5.6 .6 Fairness Index . . . . . . . . . . . . . . . . . . . . 66

5.7 Summary . . . . . . . . . . . . . . . . . . . 67

6 Conclusion and Future Work $\quad 69$

6.1 Conclusion . . . . . . . . . . . . . . . . . . . . . 69

6.2 Future Work . . . . . . . . . . . . . . . . . . . 71

6.2.1 Pushing Capacity Supply to Traffic Demand . . . . . . . . 71

6.2.2 Pushing Traffic Demand to Capacity Supply . . . . . . . . . 71

$\begin{array}{ll}\text { List of References } & 72\end{array}$ 


\section{List of Tables}

2.1 Basic statistics of distance-based measures for a PPP in one, two and three dimensions. . . . . . . . . . . . . . . . . . . . . . . 15

3.1 Path loss model . . . . . . . . . . . . . . . . . . . . . . . . . 22

3.2 Simulation parameters in Chapter 3 and Chapter 4 . . . . . . . . 22

5.1 Simulation parameters in Chapter 5 . . . . . . . . . . . . 58 


\section{List of Figures}

1.1 An example of HetNets architecture. . . . . . . . . . . . . 2

1.2 Contributions and organization $\ldots \ldots \ldots \ldots \ldots$

2.1 A realization of a PPP and its corresponding HCPP and PCP. . . . 9

2.2 An example of the intensity maps of a PPP and a LGCP . . . . . 10

2.3 An example of user distribution with different deviation in LGCP. . . 11

2.4 Voronoi and Delaunay tessellations. . . . . . . . . . . . . . . 14

3.1 Macrocell geometry. . . . . . . . . . . . . . . . . . . . . . . 19

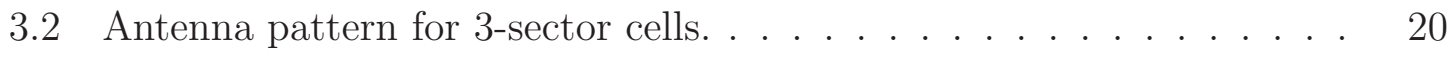

3.3 Antenna bearing orientation diagram. . . . . . . . . . . . . 21

3.4 Illustration of resource allocation scheme for finite user rate demand. 24

3.5 Mean user rate of the users in a typical cell with the proposed resource allocation scheme. . . . . . . . . . . . . . . . . . . 26

3.6 Mean user rate of the users in the system with proposed resource allocation scheme. . . . . . . . . . . . . . . . . 26 26

3.7 Mean user rate versus user spatial heterogeneity under different network configurations. . . . . . . . . . . . . . . 27

3.8 Median user rate versus user spatial heterogeneity under different network configurations. . . . . . . . . . . . . . . . 28

$3.95^{\text {th }}$ percentile of user rate versus user spatial heterogeneity under different network configurations. . . . . . . . . . . . . . . . . . . . . 29

3.10 Mean user rate versus user spatial heterogeneity under different rate limit. . . . . . . . . . . . . . . . . . . 30

4.1 An example of core, border, and noise points. . . . . . . . . 34

4.2 Example of noise points elimination in preprocessing of $k$-means. . . . 35

4.3 Example of $k$-means clustering and the splitting in postprocess. . . . 36

4.4 Example of different cluster selection criteria. . . . . . . . . . 38 
4.5 Mean user rate versus user spatial heterogeneity with different smallcell deployment strategies and different cluster selection methods. . . 40

4.6 Jain's fairness index of user rate versus user spatial heterogeneity under different network configurations. . . . . . . . . . . . . . 40

5.1 The UIL system theoretic model diagram. . . . . . . . . . . . . 45

5.2 Empirical CCDF and exponential fit for data service and suggested UIL movement with a discount and penalty surcharge incentive. . . .

5.3 Empirical CCDF and exponential fit for video service and suggested UIL movement with a discount and penalty surcharge incentive. . . . 50

5.4 An example of load balancing achieved by UIL. . . . . . . . . 55

5.5 The number of users in the system over time in one drop. . . . . . . 60

5.6 Statistics of user movement over time. . . . . . . . . . . . 61

5.7 The CoV of user spatial distribution over time with and without UIL. 62

5.8 The standard deviation of GBR user load $(\rho)$ of all the cells with respect to user spatial heterogeneities under different load balancing strategies. 63

5.9 The outage percentage of GBR users with respect to user spatial heterogeneities under different load balancing strategies. . . . . . . . . 64

5.10 Mean rate of all the GBR users in the system with respect to user spatial heterogeneities under different load balancing strategies. . . . 65

5.11 Mean rate of all the BE users in the system with respect to user spatial heterogeneities under different load balancing strategies. . . . . . 66

5.12 Jain's index of rate of all the GBR users in the system with respect to user spatial heterogeneities under different load balancing strategies. .

5.13 Jain's index of rate of all the BE users in the system with respect to user spatial heterogeneities under different load balancing strategies. . 68 


\title{
List of Abbreviations
}

\author{
BE Best Effort \\ BPP Binomial Point Process \\ BS Base Station \\ CCDF Complementary Cumulative Distribution Function \\ CDMA Code Division Multiple Access \\ $\mathrm{CoV} \quad$ Coefficient of Variation \\ COV Covariance \\ CRE Cell Range Expansion \\ DBSCAN Density-Based Spatial Clustering of Applications with Noise \\ DSPP Doubly Stochastic Poisson Process \\ FTP File Transfer Protocol \\ GBR Guaranteed Bit Rate \\ HCPP Hard Core Point Process \\ HetHetNet Network with Heterogeneous Capacity Supply and Heterogeneous Traffic De- \\ mand
}




$\begin{array}{ll}\text { HetNet } & \text { Heterogeneous Network } \\ \text { HTTP } & \text { Hypertext Transfer Protocol } \\ \text { ISD } & \text { Inter-Site Distance } \\ \text { LGCP } & \text { Log Gaussian Cox Process } \\ \text { LOS } & \text { Line-Of-Sight } \\ \text { LTE } & \text { Long Term Evolution } \\ \text { MIMO } & \text { Multiple Input Multiple Output } \\ \text { NLOS } & \text { Non-Line-Of-Sight } \\ \text { PCP } & \text { Poisson Cluster Process } \\ \text { PP } & \text { Point Process } \\ \text { PPP } & \text { Poisson Point Process } \\ \text { QoS } & \text { Quality of Service } \\ \text { SINR } & \text { Signal Interference Noise Ratio } \\ \text { UIL } & \text { User-In-the-Loop } \\ \text { UMa } & \text { Urban Macro } \\ \text { UT } & \text { User Terminal } \\ \text { WCDMA } & \text { Wideband Code Division Multiple Access } \\ & \end{array}$




\section{Chapter 1}

\section{Introduction}

\subsection{Definition}

In wireless telecommunications, "heterogeneous networks (HetNets)" refers to networks that use different types of access nodes, e.g., relays, macrocells, picocells, and femtocells (see Figure 1.1) [1] [2]. Different from the traditional tower-mounted macrocells, the distribution of small-cells ${ }^{\dagger}$ is more random and irregular. So the word "heterogeneous" is often used to refer to the non-uniform spatial distribution of wireless nodes.

In contrast, on the traffic demand side, users are assumed to be of one class (e.g., best effort) and uniformly distributed in the most literature. In this thesis, we denote the term "HetHetNet" as a cellular network with two dimensions of heterogeneity: heterogeneous capacity supply and heterogeneous traffic demand. The term "HetHetNet" was used for the first time in paper [3]. This thesis puts emphasis on the novel dimension, the demand perspective, with both the user traffic class and the user spatial distribution being heterogeneous.

\subsection{Problem Statement and Motivation}

The performance of wireless networks depends highly on their spatial configuration, not only because the signal-to-interference-plus-noise ratio (SINR) is related to the transmitter-receiver distance, but also because the traffic load in spatial domain influences the overall resource utilization, and hence, network performance. In the

${ }^{\dagger} \mathrm{WiFi}$ can be considered as one type of small-cell, nevertheless, WiFi off-loading is outside the scope of this thesis. 


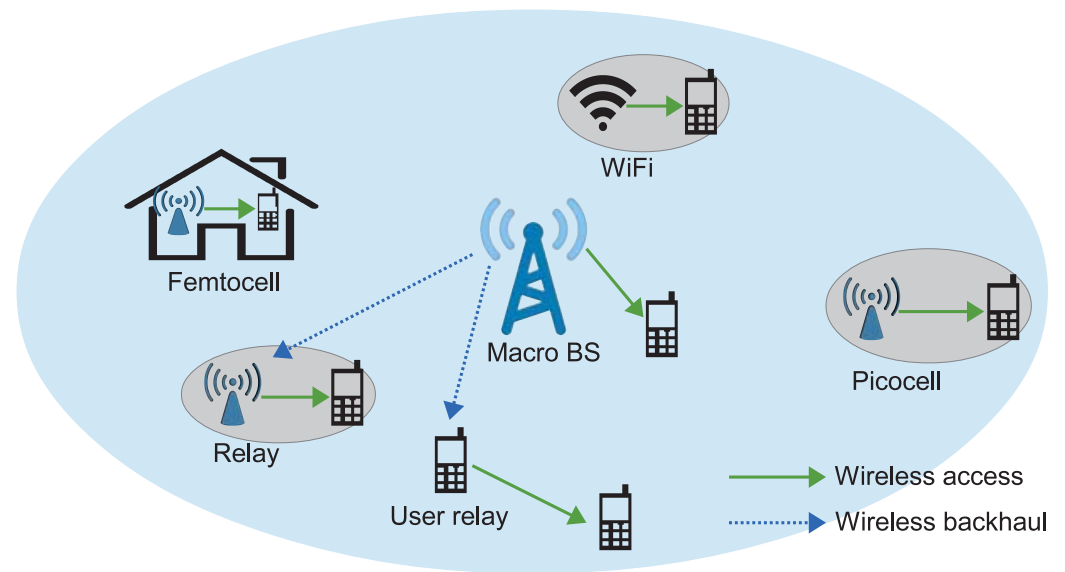

Figure 1.1: An example of HetNets architecture.

context of HetNets, the traffic load plays a more significant role in user throughput compared to the commonly used SINR metric [4]. Recently, stochastic geometry has become increasingly popular in modeling the spatial distribution of the network entities. The locations of the network entities are abstracted to a point process (PP) based on their properties. The PPs that are commonly used in wireless networks are (1) Poisson point process (PPP), (2) hard core point process (HCPP), and (3) Poisson cluster process (PCP). The PPP is the most popular PP due to its simplicity and tractability [5]. However, the research community mainly focuses on modeling the locations of base stations (BSs) rather than the users (mobile devices) [5], [6].

In the majority of the papers in wireless networks, the user spatial distribution is assumed to be random and uniform (homogeneous PPP) [6], and often with a fixed number of users, which becomes a conditional PPP, or equivalently, binomial PP (BPP) [7]. When the PPP model is used, the downlink analysis is performed at a typical user at origin according to Slivnyak's theorem [8], which states that the statistics seen from a PPP is independent from the test location.

In recent years, some papers have introduced heterogeneity to user spatial distribution. For example, in the model used in [9], the user density decreases linearly with respect to the distance from the BS up to a certain distance beyond which users are uniformly distributed to the rest of the cell area. In [10], user locations are modeled by a PCP. A parent PP $\Phi$ uniformly spreads $N_{c}$ cluster heads over the coverage region of a macrocell, and then users are dropped randomly and uniformly within a 
certain radius of each cluster head. Given a fixed total number of users, the degree of user spatial heterogeneity is controlled by changing the number of cluster heads. This method brings spatial heterogeneity to the user distribution, but due to the integer nature of the number of cluster heads, the spatial heterogeneity cannot be controlled in a continuous manner. In [11], both users and BS locations are modeled by homogeneous PPPs with different intensities in the first step, and heterogeneous user distribution is obtained by conditional thinning of BSs and the corresponding users in the Voronoi cells of BSs. This method facilitates the deduction of analytical expressions, yet the generated user distributions may not be entirely realistic because of the absence of users in the thinned areas and the identical intensity of users in the remaining areas.

The impact of non-uniform and BS-uncorrelated user distribution in a cellular CDMA network has been investigated in [12], indicating that uniform distribution can lead to an overestimation of the system capacity. In [9], the authors have shown that the performance enhances when users are concentrating around the BSs in WCDMA networks.

As such, three questions are raised: (1) How to model the heterogeneous user distribution in spatial domain (heterogeneous traffic demand)? (2) What is the impact of user spatial heterogeneity on heterogeneous cellular networks (impact of heterogeneous traffic demand on heterogeneous capacity supply)? (3) What are the solutions to combat the load imbalance between the traffic demand and the capacity supply in HetHetNets?

\subsection{Contributions and Organization}

This thesis is intended to address the three questions that are raised in the previous section with contributions and organization shown in Figure 1.2.

- First, a doubly stochastic Poisson process (DSPP), also known as the Cox process, is proposed to model the user locations. With a single parameter, the spatial heterogeneity is controlled smoothly in a broad range from uniform (PPP) to extremely heterogeneous. This is introduced in Chapter 2.

- Second, the effect of user spatial heterogeneity (captured by $C$, to be defined in Section 2.3) on the performance of downlink cellular networks is obtained. 
Chapter 2: Model user distribution

- Propose the LGCP to model user

distribution

- With a single parameter, the spatial heterogeneity is controlled smoothly in a broad range from uniform (PPP) to extremely heterogeneous
Chapter 3: Evaluation impact - Network performance deteriorates significantly with the increase of user spatial heterogeneity if the locations of users and BSs are uncorrelated - Finite user rate model
Chapter 4: Push traffic supply to demand

- Cluster analysis on the non-uniform user points is utilized to find the cluster centroids as the potential locations for small-cells - Network performance can improve with increasing user spatial heterogeneity
Matching traffic supply with traffic demand

Figure 1.2: Contributions and organization

We find that the network performance metrics deteriorate significantly when increasing $C$ if the user locations are uncorrelated with the locations of the macro and small-cell BSs. This is included in Chapter 3.

- Third, pushing capacity supply to traffic demand is the first approach we use to address the third question raised in the previous section. Cluster analysis on the non-uniform user points is utilized to find the cluster centroids as the potential locations for small-cells. Simulation results show that the network performance can improve substantially when increasing $C$ if we take advantage of user spatial heterogeneity to deploy small-cells in the appropriate locations. This is included in Chapter 4.

- Alternatively, pushing traffic demand to capacity supply is the second approach we propose to match the traffic imbalance in HetHetNets. By using the recently developed user-in-the-loop (UIL) concept, users are encouraged to move to a better location that has higher spectral efficiency and / or a lower traffic load. Based on the context, the probability of moving is taken into consideration in the utility function. Load balancing is achieved by the spatial movement of 
users which comply with the suggestions. Numerical results show that UIL can double the mean user rate in comparison to the max-SINR or the load-aware cell association strategy, and also result in a more balanced load across the network. This is included in Chapter 5.

Besides, the user traffic type is also heterogeneous in this thesis. We investigate users with finite rate demand in Chapter 3 and users with two traffic classes (best effort and guaranteed bit rate) in Chapter 5 .

The manuscripts developed (in full or in part) based on the work in this thesis are listed below:

- M. Mirahsan, Z. Wang, R. Schoenen, H. Yanikomeroglu, and M. St-Hilaire, "Unified and non-parameterized statistical modeling of temporal and spatial traffic heterogeneity in wireless cellular networks," in Proc. IEEE International Conference on Communications (ICC) Workshop on 5G Technologies, Sydney, Australia, June 2014. Section 2.3 is referenced from this paper.

- R. Schoenen, Z. Wang, H. Yanikomeroglu, G. Senarath, N. Dao, "System and Method for Wireless Load Balancing", U.S. Patent application number: 62/036,801, August 13, 2014.

- Z. Wang, R. Schoenen, H. Yanikomeroglu, and M. St-Hilaire, "The Impact of User Spatial Heterogeneity on Heterogeneous Cellular Networks," in Proc. IEEE Global Communications Conference (Globecom) Workshop on Heterogeneous and Small Cell Networks, Austin, TX, USA, Dec. 2014. The work in this paper is based on Chapters 2, 3 and 4 .

- Z. Wang, R. Schoenen, H. Yanikomeroglu, and M. St-Hilaire, "Load Balancing in Cellular Networks with User-in-the-loop: A Spatial Traffic Shaping Approach," submitted to IEEE International Conference on Communications (ICC), London, UK, June 2015. The content of this paper is based on Chapter 5.

- Z. Wang, R. Schoenen, H. Yanikomeroglu, and M. St-Hilaire, "Matching the Traffic Demand with the Traffic Suppply in HetHetNets," This the working title for a paper in progress based on this thesis aiming for a journal submission. 


\section{Chapter 2}

\section{Model for User Spatial Heterogeneity}

In this chapter, we first introduce the commonly used point processes (PPs) in the literature, and then we introduce the PP that we adopt to model the heterogeneous user distribution: the log Gaussian Cox process. The metric that is used to capture the degree of the spatial heterogeneity is introduced next.

\subsection{Point Processes in Stochastic Geometry}

In stochastic geometry analysis, the locations of the network entities are represented by a PP that captures their properties. User locations in a wireless cellular network in space domain can be modeled by a two-dimensional (2D) or three dimensional (3D) point process. A very inclusive review of point processes in space domain is conducted in [13]. Fixed distance between points generates perfect homogeneity (lattice). Poissonian distribution generates complete randomness. For generating sub-Poisson patterns, one way is to generate a perfect lattice and apply a random perturbation on its points [14] [15]. For generating super-Poisson patterns, hierarchical randomness based on doubly stochastic clustering perturbation can be used. Clustering perturbation of a given (parent) process $\Phi$ consists of independent replication and displacement of points of $\Phi$. All replicas of $x \in \Phi$ form a cluster. A survey of super-Poisson processes in space domain can be found in [13].

In this section, we first define the most popular PPs used in wireless communications, and then we propose the PP that we adopt to model the heterogeneous user spatial distribution. 


\subsubsection{Poisson Point Process}

A PP is a Poisson point process (PPP) if and only if the number of points inside any compact set $B \subset \mathbb{R}^{d}$ is a Poisson random variable, and the numbers of points in disjoint sets are independent. For example, if the average intensity is $\lambda$, the number of points in a compact set $B \subset \mathbb{R}^{d}$ is a Poisson variable with mean $\lambda \cdot L_{B}$, where $L_{B}$ is the Lebesgue measure (also called n-dimensional volume) of the set $B$. For $n=$ 1,2 , or $3, L_{B}$ coincides with the standard measure of length, area, or volume. In this thesis, the term "PPP" refers to the homogeneous PPP, in which the intensity is constant throughout the service area.

The PPP is the most popular and the most important PP in wireless communications for its simplicity, tractability, and independence property [5]. Models based on PPP have been used for packet radio networks for more than three decades [16], and the performance of PPP-based networks has been well characterized and understood [5]. Recent studies led by Professor Jeffery G. Andrews's group have used PPP to model the locations of BSs and users in HetNets [6] [17].

The PPP is important also because it is the basic model to construct more complicated point processes, such as the Matérn Hard Core Point Process (HCPP) and Poisson Cluster Process (PCP) introduced in the coming subsections.

\subsubsection{Binomial Point Process}

A PP is a Binomial point process (PPP) if and only if the number of points inside any compact set $B \subset \mathbb{R}^{d}$ is a binomial random variable, and the numbers of points in disjoint sets are related via a multinomial distribution. For example, if the total number of points in the service area is $n$ and the average intensity of points is $\lambda$, the number of points in a compact set $B \subset \mathbb{R}^{d}$ follows a Binomial distribution $b(n, p)$ with $p=\lambda \cdot L_{B}$.

Note that any realization of a finite PPP (or conditional PPP) is a BPP with the number of realized points [7]. In wireless communications, if the total number of nodes is known and the service area is finite (e.g., a fixed number of picocells are dropped in a finite service area in this thesis), then the BPP is used to model the network entities. 


\subsubsection{Hard Core Point Process}

An HCPP is a point process that no two points of the process coexist with a distance less than a hard core threshold. HCPP is derived by applying a specific thinning regarding the hardcore distance on the underlying (or parent) PP. When it is constructed from a PPP, it is called a Poisson HCPP or Matérn HCPP.

In cellular networks, the BSs and the users cannot be very close to each other, e.g., in 3GPP release 9 [18], the minimum distance of a small-cell to the existing macrocells is 75 meters, and the minimum distance between a user to a macrocell is 35 meters. In [19], Matérn HCPP is used to model the active cognitive femtocells in a two-tier HetNet.

\subsubsection{Poisson Cluster Process}

The PCP is constructed from a parent PPP by replacing each point with a cluster of points. When the cluster points are within a disc of radius (around the cluster center) and are realizations of a PPP, it is called a Matérn cluster process. For example, a Matérn cluster process can be constructed in two steps. First, generate a PPP $\Phi$ with intensity $\lambda_{0}\left(\lambda_{0}>0\right)$, and then replace each point $x_{i} \in \Phi$ with a cluster of points. The points of each cluster are within a disc of $R$ of its cluster center from the parent process $\Phi$, and the distribution of them is a realization of another PPP with intensity $\lambda_{1}\left(\lambda_{1}>0\right)$.

A PCP is used in [10] to model the users around the femtocells. First a number of femtocells are dropped in the service area using a BPP (PPP with a finite number of points), and then a fixed number (instead of Poisson variable) of users are dropped within a certain radius of the femtocells randomly and uniformly.

A realization of a PPP and its corresponding HCPP and PCP are shown in Figure 2.1 .

\subsection{Log Gaussian Cox Process}

Packet arrivals in time domain can be modeled by a one dimensional (1D) point process. A fixed inter-arrival time (IAT) between packets generates maximum homogeneity (lattice). Exponentially distributed IAT generates complete randomness (Poisson). Various models for generating super-Poisson patterns (patterns with more 


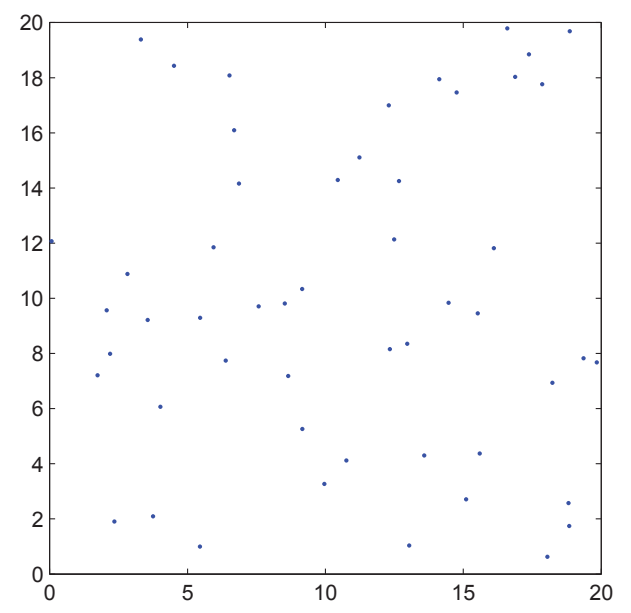

(a) PPP

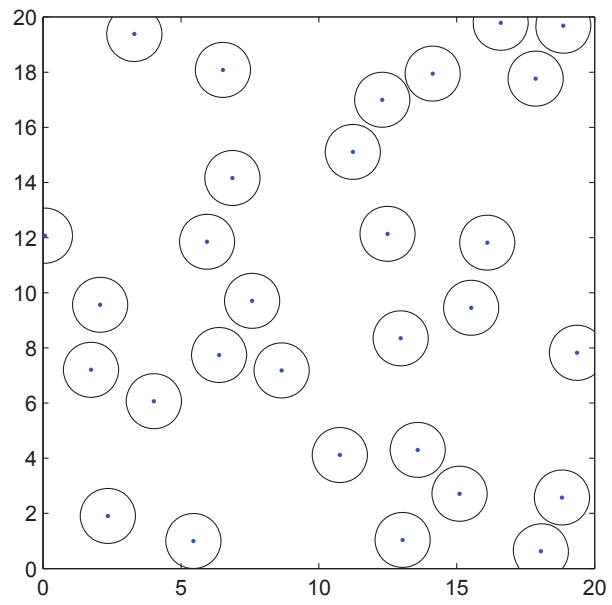

(b) HCPP

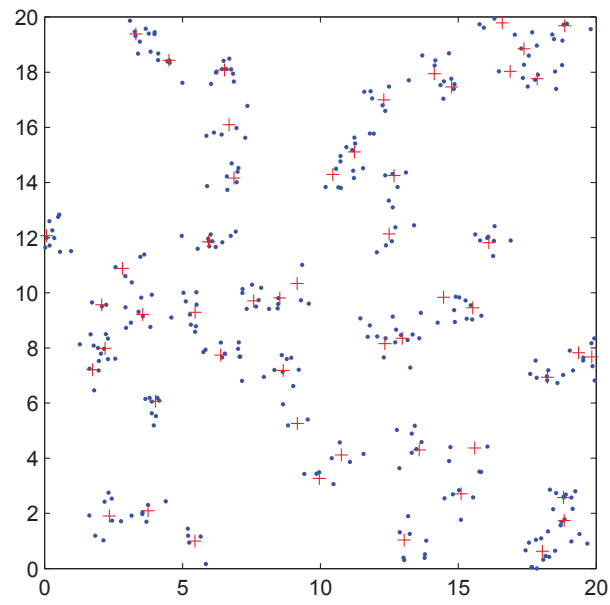

(c) PCP

Figure 2.1: A realization of a PPP and its corresponding HCPP and PCP: (a) PPP in a $20 \mathrm{~m} \times 20 \mathrm{~m}$ region with intensity 0.1 points $/ \mathrm{m}^{2}$, (b) $\mathrm{HCPP}$ in a $20 \mathrm{~m}$ $\times 20 \mathrm{~m}$ region for the parent PPP in (a) and hard core parameter $r=1 \mathrm{~m}$, each point of the HCPP lies at the center of a non-overlapping circles with radius $r$ represented by the circles, (c) PCP in a $20 \mathrm{~m} \times 20 \mathrm{~m}$ region for the parent PPP in (a) and clusters with a Poisson distributed number of points with mean 2 uniformly distributed in a unit circle neighborhood (i.e., Matérn cluster process), the parent PPP points are plotted with crosses ("+") while the added cluster points are plotted with dots. 


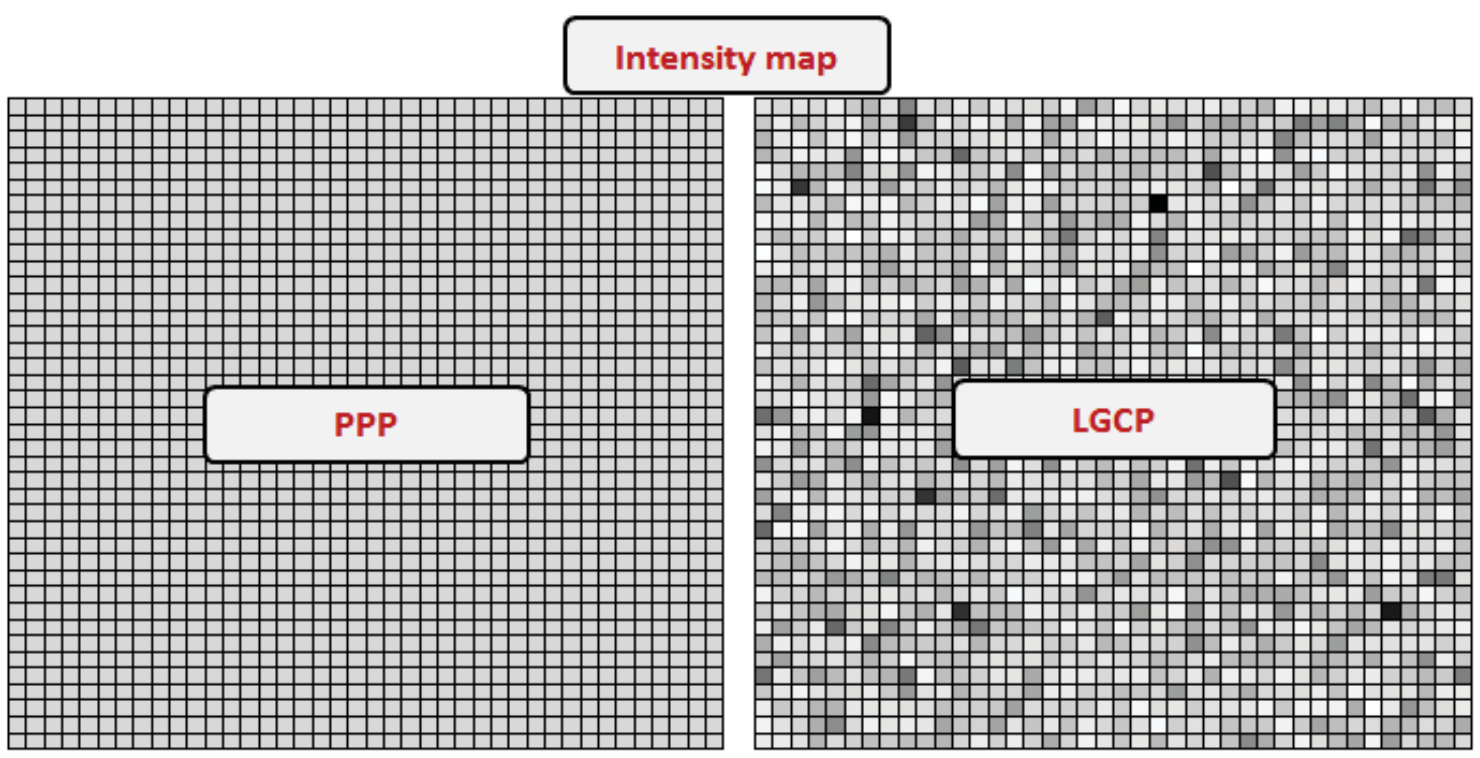

Figure 2.2: An example of the intensity maps of a PPP and a LGCP

heterogeneity than Poisson) have been proposed in the literature which are mostly based on hierarchical randomness and Markov models [20] [21] [22].

In this thesis, we propose to use the Cox process, a generalization of the PPP, to model the spatial heterogeneity of the user distribution. Instead of being constant as in PPP, the intensity in Cox is itself a stochastic process [8]. An example of the intensity maps of a PPP and a LGCP is shown in Figure 2.2. It is first studied by Cox under the name doubly stochastic Poisson process (DSPP), for the reason that it can be viewed as a two randomization procedure. A process $\Lambda$ is used to generate another process $\Phi$ by acting as its intensity, which means that $\Phi$ is a PPP conditional on $\Lambda$ (if $\Lambda$ is deterministic, then $\Phi$ is a PPP). For example, in a homogeneous PPP with intensity $\lambda$, the number of points in a bounded Borel set $B \subset \mathbb{R}^{2}$ is Poisson distributed with mean $\lambda \cdot A_{B}$, where $A_{B}$ is the area of $B$. While in the Cox process, the number of points in $B$ is Poisson distributed with a mean intensity $\bar{\Lambda}=\int_{B} \Lambda(s) d s, s \in B$, where $\Lambda(\cdot)$ is an intensity function. From the definition, and also as the name DSPP implies, Cox process brings a second layer of randomness to the Poisson process by generalizing the constant intensity $\lambda$ into a intensity function $\Lambda(\cdot)$. By varying $\Lambda(\cdot)$, we get different kinds of Cox processes.

A Cox process is called a log Gaussian Cox process (LGCP) if $\Lambda(s)=\exp \{Y(s)\}$, 

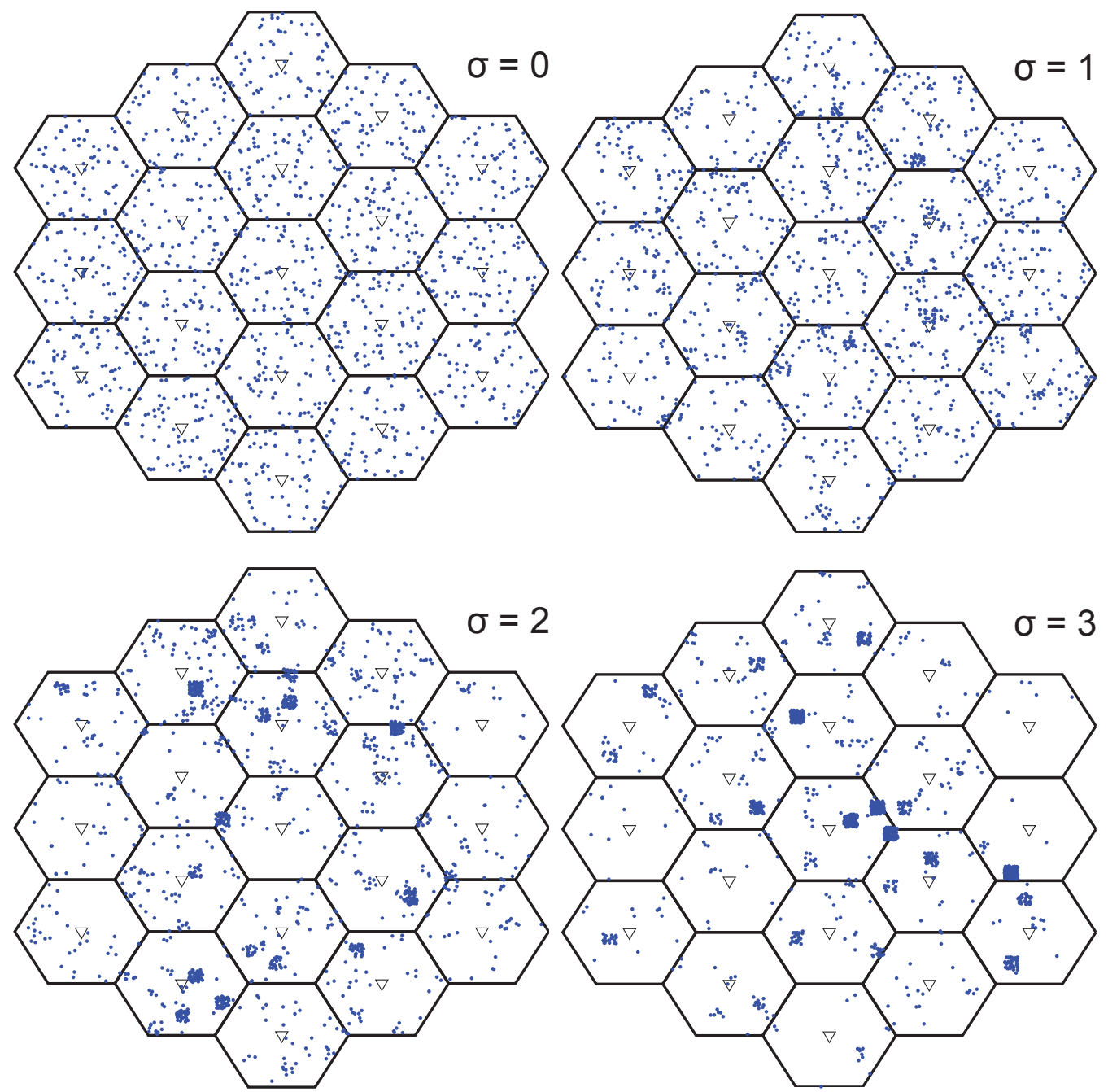

Figure 2.3: An example of user distribution with different deviation in LGCP. The PPP is the special case of LGCP (when $\sigma$ equals to zero). Each dot represents an active user in the system. With the measure that will be discussed in Section 2.3, the degree of spatial heterogeneity $C$ equals to $1.00,1.44,2.70,4.72$, respectively.

where $Y=\left\{Y(s): s \in \mathbb{R}^{2}\right\}$ is a real-valued Gaussian process (i.e., the joint distribution of any finite vector $\left(Y\left(s_{1}\right), \ldots, Y\left(s_{n}\right)\right)$ is Gaussian) [23]. The distribution of $Y$ is specified by the mean $\mu=E(Y(s))$, the variance $\sigma^{2}=\operatorname{Var}(Y(s))$, and the covariance $\operatorname{COV}\left(Y\left(s_{i}\right), Y\left(s_{j}\right)\right)^{\dagger}$. In this thesis, we assume $C O V\left(Y\left(s_{i}\right), Y\left(s_{j}\right)\right)=0$ for $i \neq j$, indicating no correlation within $\Lambda(\cdot)$.

${ }^{\dagger}$ The $C O V$ used here for "covariance" should not be confused with CoV used for "coefficient of 
The distribution of $\Lambda(\cdot)$ is specified by the mean $m=\exp \left(\mu+\sigma^{2} / 2\right)$ and the variance $v=\exp \left(2 \mu+\sigma^{2}\right)\left(\exp \left(\sigma^{2}\right)-1\right)$. Due to the exponential nature of the intensity function $\Lambda(\cdot)$, the LGCP provides a wide range of intensity values with a small variation in $\sigma$. When $\sigma$ is equal to zero, $\Lambda$ becomes constant, and the LGCP falls back to a homogeneous PPP. By increasing $\sigma$ (and changing $\mu$ accordingly to keep the overall user intensity $m$ constant), the intensity $\Lambda$ becomes more fluctuating (higher $v$ ), resulting in higher spatial heterogeneity over the whole area. A realization of LGCP with different $\sigma$ values is shown in Figure 2.3. Note that $\sigma$ can take any nonnegative real value continuously from 0 to infinity, which facilities the smooth control of the user spatial heterogeneity.

The implementation of LGCP involves two steps. First the Gaussian field is generated in the minimum square that contains the coverage area of all the cells (in this thesis, the 19 hexagons). We adopt the method in [23] by discretizing the square into tiles ${ }^{\ddagger}$ and approximating the Gaussian process by the values of the corresponding Gaussian distribution on the tiles. Then the Gaussian field $\tilde{Y}=\left(\tilde{y}_{i j}\right)_{(i, j \in I)}$ is obtained, where $I$ represents all the tiles after discretization. In the second step, for the given Gaussian field $\tilde{Y}$, a homogeneous PPP with intensity $\tilde{\lambda}=\exp \left(\tilde{y}_{i j}\right)$ is generated in each tile.

\subsection{Measure of User Spatial Heterogeneity}

Before we evaluate the network performance with respect to adjustable user spatial heterogeneity, we need a statistical measure to capture the degree of spatial heterogeneity. This section introduces the method first proposed in my previous co-authored work [24], in which measures based on the Voronoi and Delaunay tessellations are used.

variation" defined in Section 2.3.

$¥$ A coarse discretization results in a small sample size, and hence a decreased statistical variation, while a refined discretization may result in an unrealistic situation (e.g., many users squeezed in a small area) and higher computational complexity. For the simulations in this thesis, we find that it is sufficient to use $40 \times 40$ tiles (i.e., a total number of 1600 tiles for the square enclosing area). 


\subsubsection{Related Work}

A 1D point process in time domain can be measured mathematically in many different ways. One may use the interval counts $N(a ; b]=N_{b}-N_{a}$ which is a densitybased metric and divide the whole domain into smaller windows and count the number of process points in each window. A disadvantage of density-based metrics is that they are parameterized by the window size. Finding an appropriate window size is itself a challenging question and cannot be answered generally for all applications. Interarrival time $I_{i}=T_{i+1}-T_{i}$ is the most popular and best-accepted metric because it is distance-based rather than density-based and considers the distance between every two neighboring points in domain. Considering $\mathrm{CoV}(C)$, the normalized second-order statistic, defined as the ratio of standard deviation to the mean, for 1D-lattice, the constant IAT has $C_{I}=0$. For a 1D-Poisson pattern, $C_{I}=1$ since for an exponential distribution with parameter $\lambda$ the standard deviation and the mean are both $\mu_{I}=$ $\sigma_{I}=\lambda$. Sub-Poisson processes have $0<C_{I}<1$ and super-Poisson processes have $C_{I}>1$.

As mentioned above, in time domain, the inter-arrival time captures heterogeneity by one nonparameterized real value $C_{I}$. In multi-dimensions, however, there is no natural ordering of the points, so finding the analogue of the inter-arrival time is not easy. There are many density-based heterogeneity metrics in the literature like Ripleys K-function and pair correlation function [13] but they are all parameterized. For introducing distance-based metrics, the problem is about defining the next point or the neighboring points in multi-dimensional domains. The first and the simplest candidate for characterizing neighboring points in multi-dimensional domain is the nearest-neighbor. This leads to the nearest-neighbor distance metric [25]. However, the nearest-neighbor distance metric in 1D time domain is not the analogue of the inter-arrival time because it is considering the $\min \left(I_{i} ; I_{i}+1\right)$ for every point $T_{i}$. So the nearest neighbor fails to capture the process statistics in multi-dimensional domains because it only considers the nearest neighbor and ignores the other neighbors. The next candidate is the distance to the $k_{t h}$ neighbor. However, determining $k$ globally is not possible because every point may have different number of neighbors. All the above mentioned metrics either require a parameter (the setting of which is itself a question), or do not capture the statistics in a proper way. So we need a nonparameterized metric that can measure the spatial heterogeneity in multi-dimensional domain like the inter-arrival time in one-dimensional time domain. 


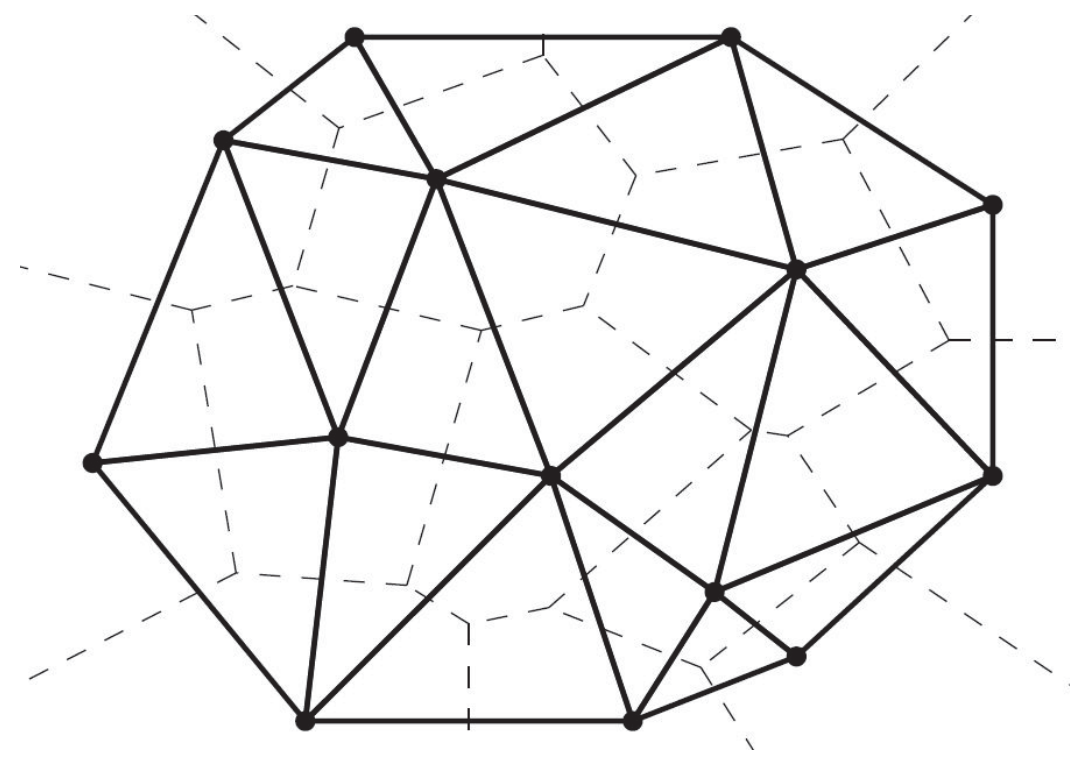

Figure 2.4: Voronoi (dashed lines) and Delaunay (solid lines) tessellations [24].

\subsubsection{Unified and Nonparameterized Metrics}

Given a point pattern $P=\left\{p_{1}, p_{2}, \ldots, p_{n}\right\}$ in d-dimensional space $\mathbb{R}^{d}$, the Voronoi tessellation $T=\left\{C_{p 1}, C_{p 2}, \ldots, C_{p n}\right\}$ is the set of cells such that every location, $y \in c_{p i}$, is closer to $p_{i}$ than any other point in $P$. This can be expressed formally as

$$
c_{p i}=\left\{y \in \mathbb{R}^{d}:\left|y-p_{i}\right| \leq\left|y-p_{j}\right| \text { for } i, j \in 1, \ldots, n\right\}
$$

The Voronoi tessellation in $\mathbb{R}^{d}$ has the property that each of its vertices is given by the intersection of exactly $d+1$ Voronoi cells. The corresponding $d+1$ points define a Delaunay cell. So the two tessellations are said to be dual. Figure 2.4 demonstrates a pattern of points with its Voronoi tessellation (dashed lines) and Delaunay tessellations (solid lines).

Every two points sharing a common edge in the Voronoi tessellation or equivalently every two connected points in the Delaunay tessellation of a point process are called natural neighbors. This gives an inspiration of neighboring relation in multidimensional domains and leads us to the well accepted inter-arrival time metric in multi-dimensions. Various statistical inferences based on different properties of cells generated by these tessellations can be considered for measurement of a point pattern.

Voronoi Cell Area or Voronoi Cell Volume $V$ is the first natural choice. For a 
Table 2.1: Basic statistics of distance-based measures for a PPP in one, two and three dimensions [24]. $\lambda$ is the exponential distribution parameter for interarrival time and $\Lambda$ is the mean intensity of point processes.

\begin{tabular}{|l|l|l|l|l|}
\hline Distance-based measures & Statistics & $1 \mathrm{D}$ & $2 \mathrm{D}$ & $3 \mathrm{D}$ \\
\hline \multirow{4}{*}{ Nearest-neighbor distance $(\mathrm{G})$} & Mean $(\mu)$ & $0.5 \lambda^{-1}$ & $0.5 \Lambda^{-0.5}$ & $0.5539 \Lambda^{-0.33}$ \\
& Variance $\left(\sigma^{2}\right)$ & $0.25 \lambda^{-2}$ & $0.0683 \Lambda^{-1}$ & $0.04 \Lambda^{-0.66}$ \\
& $\mathrm{CoV}(C)$ & 1 & 0.653 & 0.364 \\
\hline \multirow{4}{*}{ Voronoi cell area/volume (V) } & Mean $(\mu)$ & $\lambda^{-1}$ & $\Lambda^{-1}$ & $\Lambda^{-1}$ \\
& Variance $\left(\sigma^{2}\right)$ & $\lambda^{-2}$ & $0.28 \Lambda^{-2}$ & $0.18 \Lambda^{-2}$ \\
& $\operatorname{CoV}(C)$ & 1 & 0.529 & 0.424 \\
\hline \multirow{5}{*}{ Delaunay cell edge length $(\mathrm{E})$} & $\operatorname{Mean}(\mu)$ & $\lambda^{-1}$ & $1.131 \Lambda^{-0.5}$ & $1.237 \Lambda^{-0.33}$ \\
& $\operatorname{Variance}\left(\sigma^{2}\right)$ & $\lambda^{-2}$ & $0.31 \Lambda^{-1}$ & $0.185 \Lambda^{-0.66}$ \\
& $\operatorname{CoV}(C)$ & 1 & 0.492 & 0.347 \\
\hline
\end{tabular}

lattice process, all the cell areas in 2D or cell volumes in $3 \mathrm{D}$ are equal and $\mathrm{CoV}=$ 0. The statistics of the Voronoi cells for a Poisson point process (Poisson-Voronoi Tessellation) are well investigated in the literature [26-30]. Square rooted Voronoi cell area in 2D or cube rooted Voronoi cell volume in 3D can also be considered.

The next proposed metric is the Delaunay edge length $E$. The statistics for the Delaunay tessellations are investigated in [31-33]. The mean value of the lengths of Delaunay edges of every point $M$ can also be considered.

A Delaunay tessellation divides the space to triangles or tetrahedrons in 2D and $3 \mathrm{D}$, respectively. The area distribution of the triangles or the volume distribution of tetrahedrons $T$ can determine the properties of the underlying pattern.

Voronoi and Delaunay tessellations can be applied on a 1D process which models traffic in time domain. In this case, the introduced distance-based metrics are converted to time domain metrics. Basic statistics of these metrics for a Poisson point process in one, two and three dimensions are summarized in Table 2.1.

In order to use the above mentioned metrics as an analogue of inter-arrival time, one needs to normalize their $\mathrm{CoV}$ to the $\mathrm{CoV}$ values of inter-arrival time in the time domain. For the complete homogeneity case, the $\mathrm{CoV}$ values are already zero 
like inter-arrival time. To normalize the $\mathrm{CoV}$ values of complete random case to 1, it is required to divide the metrics by the values presented in Table 2.1. After normalization, $C$ is larger than 1 in super-Poisson processes, equal to 1 in PPP, and between 0 and 1 in sub-Poisson processes. The LGCP introduced in the previous section brings more heterogeneity to the PPP, so it is super-Poisson processes with $C$ equal to or greater than 1. For example, for the user distributions in Figure 2.3, we first draw the Voronoi tessellations for the user points, and measure the area of each Voronoi cell, $A_{i}$. Then the $\mathrm{CoV}$ of $A$ is calculated from the ratio of the standard deviation and the mean of $A$. Finally, the spatial heterogeneity level $C$ is obtained from the normalized $\mathrm{CoV}$.

\subsection{Summary}

In this chapter, we propose the Cox process, a generalization of the PPP, to model the spatial heterogeneity of the user distribution. Instead of being constant as in PPP, the intensity in Cox is itself a stochastic process [8]. By varying the intensity function, we get different kinds of Cox processes. To get a broad range of heterogeneity of intensity, we propose to use the log Gaussian Cox process to model the user spatial heterogeneity. With a single parameter, the spatial heterogeneity is controlled smoothly in a broad range from uniform (PPP) to extremely heterogeneous.

To measure the degree of spatial heterogeneity, this thesis adopts the method introduced in my previous co-authored work [24], in which measures based on the Voronoi and Delaunay tessellations are proposed, and coefficient of variation, the normalized second-order statistic (the standard deviation divided by the mean), is suggested to be used to capture the main statistical properties of the measures. The statistics of PPP are used as the normalization factors to normalize those of the subPoisson processes and super-Poisson processes. Then, the user spatial heterogeneity can be represented by a non-negative real number $C$, the normalized $\mathrm{CoV}$ of different measures (e.g., the Voronoi cell area used in this thesis). Based on the formulation, $C$ is greater than 1 in super-Poisson processes, equal to 1 in PPP, and between 0 and 1 in sub-Poisson processes. The LGCP introduces more heterogeneity in PPP, so it is a super-Poisson point process in which PPP constitutes a special case (when $\sigma=0$ ). 


\section{Chapter 3}

\section{The Impact of User Spatial Heterogeneity}

In this chapter, we use the user spatial model discussed in the previous chapter to generate the heterogeneous user distributions (independently with the locations of BSs), and evaluate the network performance with respect to the degree of user spatial heterogeneity $(C)$ under different network configurations. More precisely, the guidelines of performance evaluation from ITU is introduced in the first section, and the finite user rate demand model with the corresponding resource allocation scheme is discussed in the next section. The performance evaluation comes next with the impact of user spatial heterogeneity and the impact of finite rate demand evaluated respectively.

\subsection{Guidelines of Performance Evaluation}

A static snapshot-based system-level simulator is used. The system simulation parameters for performance evaluation in this thesis mainly follows the guidelines in ITU-R report M.2135 [34], the guidelines for evaluation of radio interface technologies for IMT-Advanced. As the parameters for small-cells are not available in this ITU guidelines, this thesis also opts for the settings in 3GPP release 9 [18] for small-cell parameters. Matlab is the software we use in our simulation.

The following principles are followed in the system simulations:

- Users are dropped independently over predefined area of the network layout. The distribution of users is modeled from homogeneous to extremely heterogeneous. Each mobile corresponds to an active user session that runs for the duration of the drop. 
- Users are randomly assigned line-of-sight (LOS) and non-line-of-sight (NLOS) channel conditions based on LOS probability, which is a function of BS-user distance.

- Each user associate with one cell as serving cell, regrading to the cell selection scheme specified in the thesis.

- For a given drop, the simulation is run and then the process is repeated with the users dropped at new random locations. A sufficient number of drops are simulated to ensure the convergence in the user and system performance metrics.

- Performance statistics are collected taking into account the wrap-around configuration in the network layout to eliminate the boundary effect.

- As long as a cell (macro or small-cell) is serving one or more users, it is assumed that this cell is contributing to interference at the full transmit power level to other cells in the downlink.

The simulation of the system behaviour is carried out as a sequence of drops, where a drop is defined as one simulation run over a certain time period. A drop (or snapshot) is a simulation entity where the random properties of the channel remain constant. In a simulation, the number of drops have to be selected properly by the evaluation requirements and the deployed scenario. Sufficient drops are needed to get statistically representative results. Consecutive drops are independent.

\subsubsection{Network Layout}

The deployment scenario is based on the urban macrocell scenario described in ITU-R M.2135 [34], in which the locations of the macrocell sites are fixed and form a hexagonal grid layout as shown in Figure 3.1. 19 sites, each with 3 cells, with inter-site distance (ISD) of 500 meters, are configured in the system. The wraparound technique is applied in the simulations to eliminate the boundary effect. The HetNets consist of two tiers with small-cells (not shown in Figure 3.1) overlaid on the same area of macrocells. The macrocells adopt directional antennas while small-cells use omni antennas. The distribution of small-cells is either according to a BPP or user-distribution related. 

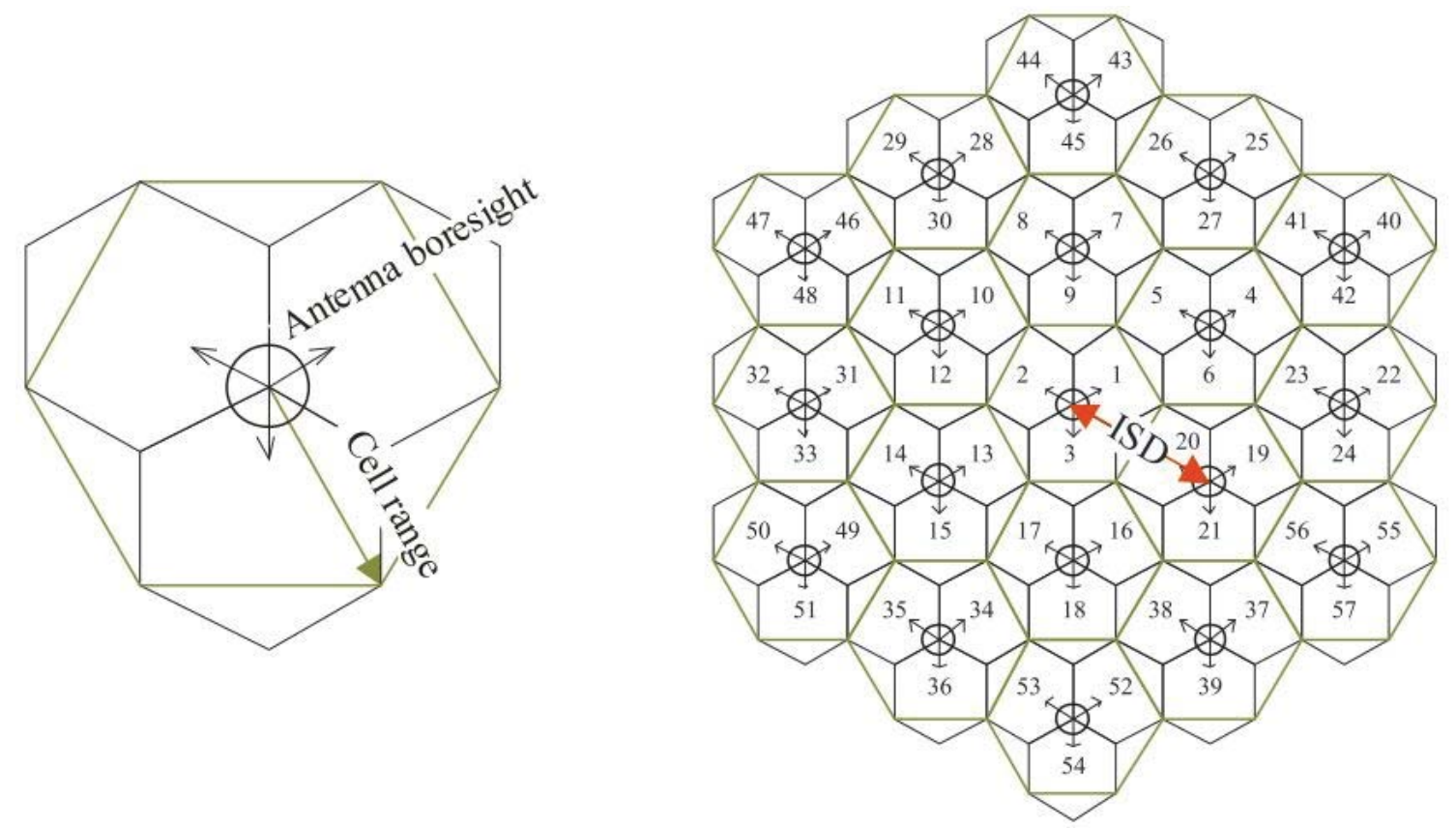

Figure 3.1: Macrocell geometry: A total number of 19 sites and 57 cells [34].

In typical urban macrocell scenario, the mobile station is located outdoors at street level and the fixed base station clearly above the surrounding building heights. The channel model for urban macrocell scenario is called urban macro (UMa) [34].

\subsubsection{Antenna Characteristics}

This subsection specifies the antenna characteristics, e.g. antenna pattern, gain, side-lobe level, orientation, etc., for antennas at the BS and the user terminal (UT), which shall be applied for the evaluation in the deployment scenarios with the hexagonal grid layout.

The horizontal antenna pattern used for each BS sector ${ }^{\dagger}$ is specified as

$$
A(\theta)=-\min \left[12\left(\frac{\theta}{\theta_{3 \mathrm{~dB}}}\right)^{2}, A_{m}\right]
$$

where $A(\theta)$ is the relative antenna gain $(\mathrm{dB})$ in the direction $\theta, 180^{\circ} \leq \theta \leq 180^{\circ}$, and $\theta_{3 \mathrm{~dB}}$ is the $3 \mathrm{~dB}$ beamwidth, and $A_{m}=20 \mathrm{~dB}$ is the maximum attenuation. Figure

\footnotetext{
${ }^{\dagger} \mathrm{A}$ sector is equivalent to a cell in this thesis.
} 


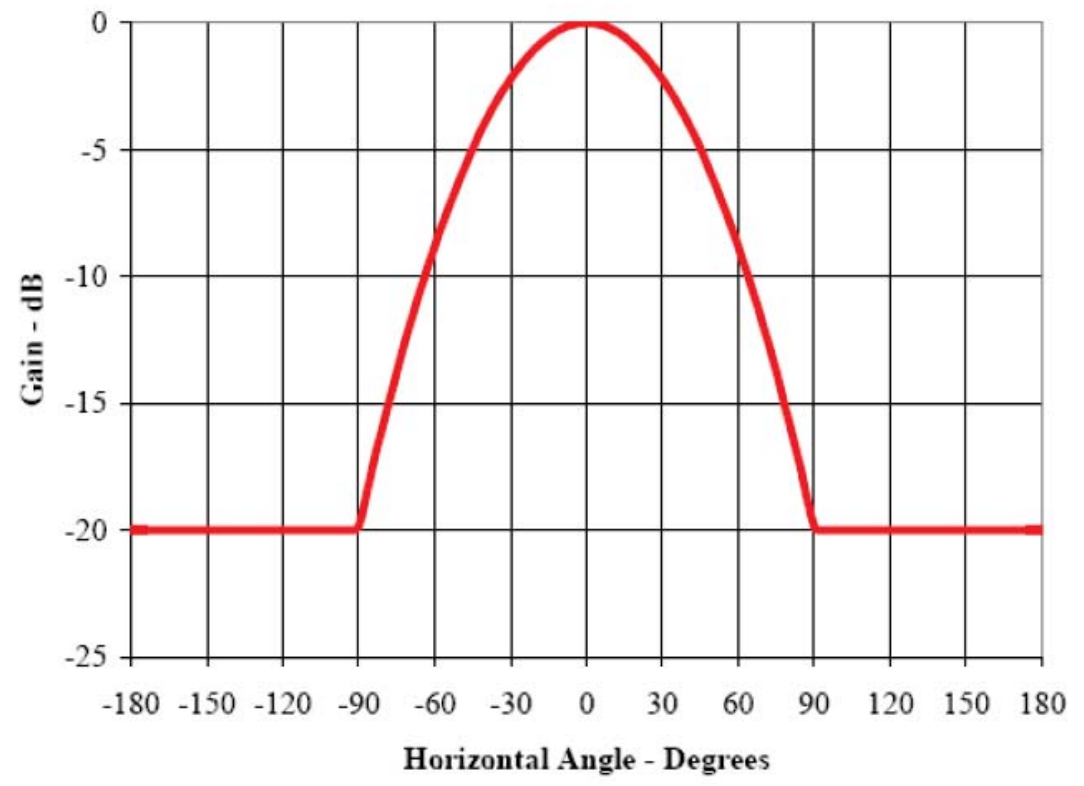

Figure 3.2: Antenna pattern for 3-sector cells [34].

3.2 shows the BS antenna pattern for 3 sector cells to be used in the system level simulations.

A similar antenna pattern will be used for elevation in simulations that need it. In this case, the antenna pattern will be given by:

$$
A_{e}(\phi)=-\min \left[12\left(\frac{\phi-\phi_{\mathrm{tilt}}}{\phi_{3 \mathrm{~dB}}}\right)^{2}, A_{m}\right] \text {, }
$$

where $A_{e}(\phi)$ is the relative antenna gain $(\mathrm{dB})$ in the elevation direction, $\phi, 90^{\circ} \leq$ $\phi \leq 90^{\circ}$, and $\phi_{3 \mathrm{~dB}}$ is the elevation $3 \mathrm{~dB}$ value. $\phi_{\text {tilt }}$ is the tilt angle. The combined antenna pattern at angles off the cardinal axes is computed as

$$
-\min \left[-\left(A(\theta)+A_{e}(\phi)\right), A_{m}\right]
$$

The antenna bearing is defined as the angle between the main antenna lobe center and a line directed east given in degrees. The bearing angle increases in a clockwise direction. Figure 3.3 shows the hexagonal cell and its three sectors with the antenna bearing orientation proposed for the simulations. The center directions of the main 


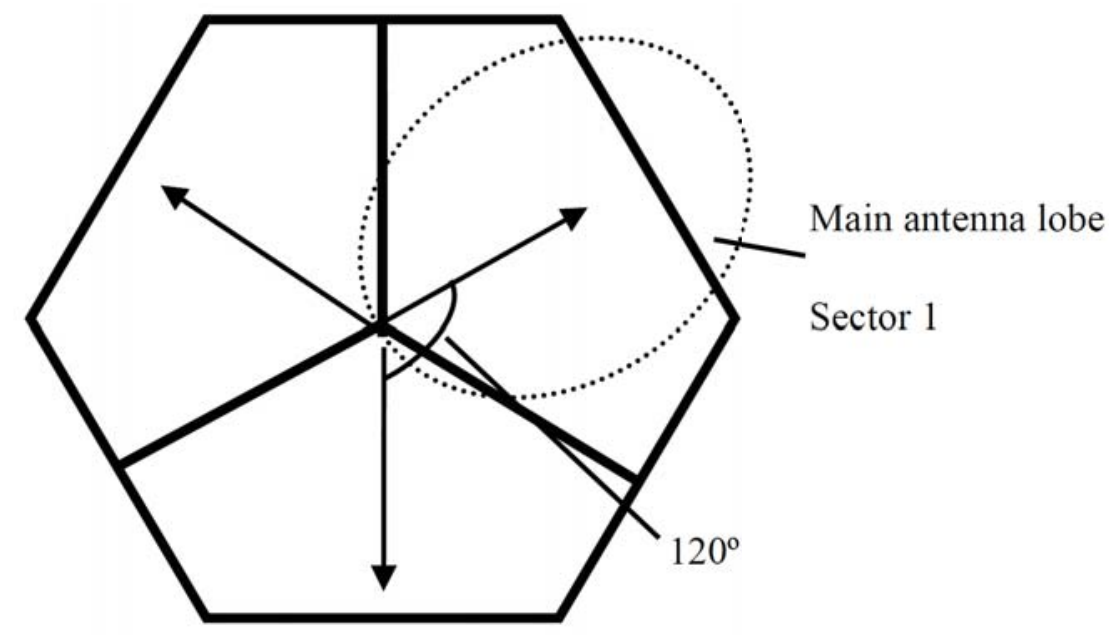

Figure 3.3: Antenna bearing orientation diagram. [34].

antenna lobe in each sector points to the corresponding side of the hexagon. The UT antenna is assumed to be omni directional.

\subsubsection{Channel Model}

The downlink signal experiences path loss and shadowing, and the fast fading is assumed to be averaged out. For macrocell-only netwroks, the path loss model is the UMa model from ITU-R M.2135 [34], which is shown in Table 3.1. The probability of LOS is calculated as

$$
P_{L O S}=\min (18 / d, 1) \times(1-\exp (-d / 36))+\exp (-d / 36),
$$

where $d$ is the distance between the BS and the UT in meters. The lognormal shadowing is used, and the standard deviation $\sigma$ in the Gaussian distributed random variable is defined in Table 3.1.

For HetNets, the path loss model is based on the 3GPP model 2 in depolyment scenario case 6.2 [18], in which outdoor picocells are the small-cells that are layered on macrocells to cover the hot-spots. Table 3.2 shows the key parameters used in the

(1) $d_{B P}^{\prime}$ is the break point distance. $d_{B P}^{\prime}=4 h_{B S}^{\prime} h_{U T}^{\prime} f_{c} / c$, where $f c$ is the center frequency $(\mathrm{Hz}), c$ is the propagation velocity in free space, and $h_{B S}^{\prime}$ and $h_{U T}^{\prime}$ are the effective antenna heights at the BS and the UT, respectively. 
Table 3.1: Path loss model [34]

\begin{tabular}{|c|c|c|c|c|}
\hline \multicolumn{2}{|c|}{ Scenario } & $\begin{array}{l}\text { Path loss }(\mathrm{dB}) \\
\text { Note: } f_{\mathrm{c}} \text { is given in } \mathrm{GHz} \text { and distance in } \mathrm{m} !\end{array}$ & $\begin{array}{l}\text { Shadow } \\
\text { fading std }\end{array}$ & $\begin{array}{l}\text { Applicability range, antenna } \\
\text { height default values }\end{array}$ \\
\hline \multirow[b]{2}{*}{ 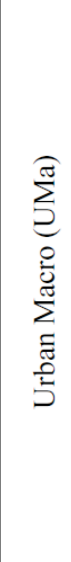 } & LoS & $\begin{aligned} P L= & 22.0 \log _{10}(d)+28.0+20 \log _{10}\left(f_{c}\right) \\
P L= & 40.0 \log _{10}\left(d_{1}\right)+7.8-18.0 \log _{10}\left(h^{\prime}{ }_{B S}\right) \\
& -18.0 \log _{10}\left(h^{\prime}{ }_{U T}\right)+2.0 \log _{10}\left(f_{c}\right)\end{aligned}$ & $\begin{array}{l}\sigma=4 \\
\sigma=4\end{array}$ & $\begin{array}{l}10 \mathrm{~m}<d<d_{B P}^{\prime}(1) \\
d_{B P}^{\prime}<d<5000 \mathrm{~m}^{(1)} \\
h_{B S}=25 \mathrm{~m}^{(1)}, h_{U T}=1.5 \mathrm{~m}^{(1)}\end{array}$ \\
\hline & NLoS & $\begin{array}{l}P L=161.04-7.1 \log _{10}(W)+7.5 \log _{10}(h) \\
-\left(24.37-3.7\left(h / h_{B S}\right)^{2}\right) \log _{10}\left(h_{B S}\right) \\
+\left(43.42-3.1 \log _{10}\left(h_{B S}\right)\right)\left(\log _{10}(d)-3\right)+ \\
20 \log _{10}\left(f_{c}\right)-\left(3.2\left(\log _{10}\left(11.75 h_{U T}\right)\right)^{2}-4.97\right)\end{array}$ & $\sigma=6$ & $\begin{array}{l}10 \mathrm{~m}<d<5000 \mathrm{~m} \\
h=\text { avg. building height } \\
W=\text { street width } \\
h_{B S}=25 \mathrm{~m}, h_{U T}=1.5 \mathrm{~m}, \\
W=20 \mathrm{~m}, h=20 \mathrm{~m} . \\
\text { The applicability ranges: } \\
5 \mathrm{~m}<h<50 \mathrm{~m} \\
5 \mathrm{~m}<W<50 \mathrm{~m} \\
10 \mathrm{~m}<h_{B S}<150 \mathrm{~m} \\
1 \mathrm{~m}<h_{U T}<10 \mathrm{~m}\end{array}$ \\
\hline
\end{tabular}

Table 3.2: Simulation parameters in Chapter 3 and Chapter 4

\begin{tabular}{|c|c|}
\hline Parameter & Assumption \\
\hline Macrocell layout & Hexagonal grid of $19 \times 3=57$ macrocells with wrap-around \\
\hline ISD & $500 \mathrm{~m}$ \\
\hline Picocell layout & 1 or 2 picocells per macrocell, BPP or user distribution related \\
\hline Average user density & $25 \mathrm{users} /$ macrocell \\
\hline System bandwidth & $10 \mathrm{MHz}$ (FDD) at $2 \mathrm{GHz}$ \\
\hline Shadowing & Log-normal, s.d. 4 for LOS, 6 for NLOS \\
\hline Macrocell Tx power & $46 \mathrm{dBm}$ \\
\hline Picocell Tx power & $37 \mathrm{dBm}$ \\
\hline Antenna gain & Macrocell: $17 \mathrm{dBi}$, picocell: $5 \mathrm{dBi}$ \\
\hline CRE biasing value & $2 \mathrm{~dB}$ \\
\hline Traffic model & Full buffer or finite rate demand \\
\hline
\end{tabular}

simulations for Chapter 3 and Chapter 4. 


\subsection{Finite User Rate Demand and Resource Allo- cation Scheme}

Users are assumed to have best effort service in the vast majority of the papers when using static snapshot-based simulations, and correspondingly, BSs are assumed to serve with full buffer. This assumption can be reasonable when users are homogeneously distributed. However, when users are heterogeneously distributed, chances are that some cells will only serve a few users. As a result, these users will reach very high data rate due to the abundance of available resources. This will affect the metrics like the sum or the mean user rate as the unrealistic high rate of a small portion of users will increase the total or mean value significantly. Besides, users have finite rate demand in reality; one user in a cell will not necessarily consume all the channels.

Sophisticated traffic models are available in the literature, however, as time is involved in the model, a more complicated dynamic event-driven simulation platform is needed. As this chapter investigates the influence of spatial traffic (user distribution) instead of temporal traffic on network performance, a time-free traffic model and the corresponding resource allocation scheme are needed.

We assume all users have the same finite rate demand, $r_{d}$. Future models may consider it as a variable with different characteristics based on the nature of different classes of traffic. Suppose $n$ users in a cell with spectral efficiency $s_{i}$, so the resource needed for each user to reach the rate $r_{d}$ is $r_{d} / s_{i}$. When $r_{d}$ is small, the total resource needed $\sum_{i}^{n} \frac{r_{d}}{s_{i}}$ is lower than the available resource $W$. This status is called the underload region. When $r_{d}$ increases, total resource needed $\sum_{i}^{n} \frac{r_{d}}{s_{i}}$ is getting closer to the available resource $W$. The first saturation point shows when all resources are allocated, which relates to the equal rate resource allocation strategy in best effort situation. In equal rate resource allocation scheme, resource allocated to user $i$ with spectral efficiency $s_{i}$ is

$$
w_{i}=\frac{W}{s_{i} \cdot \sum_{i}^{n} \frac{1}{s_{i}}},
$$

and the rate reached by all users is

$$
r=\frac{W}{\sum_{i}^{n} \frac{1}{s_{i}}} .
$$




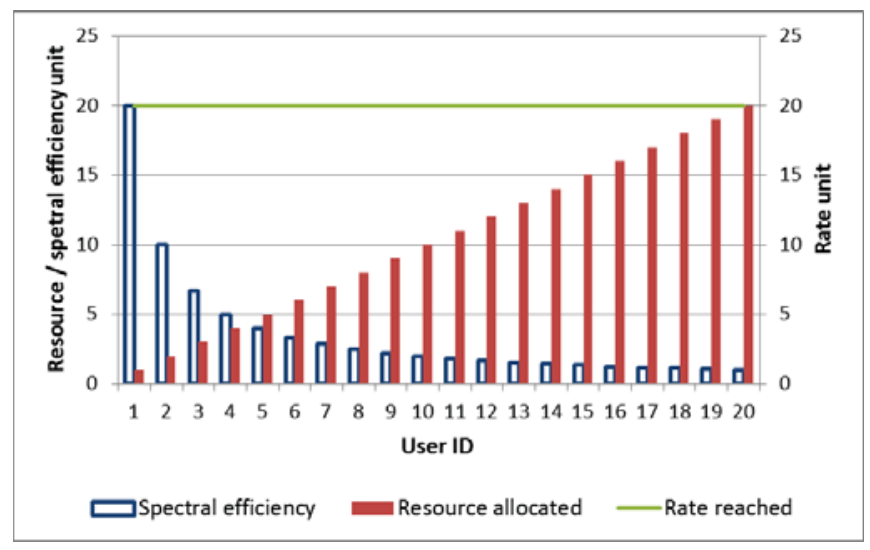

(a) Resource allocation at first saturation point

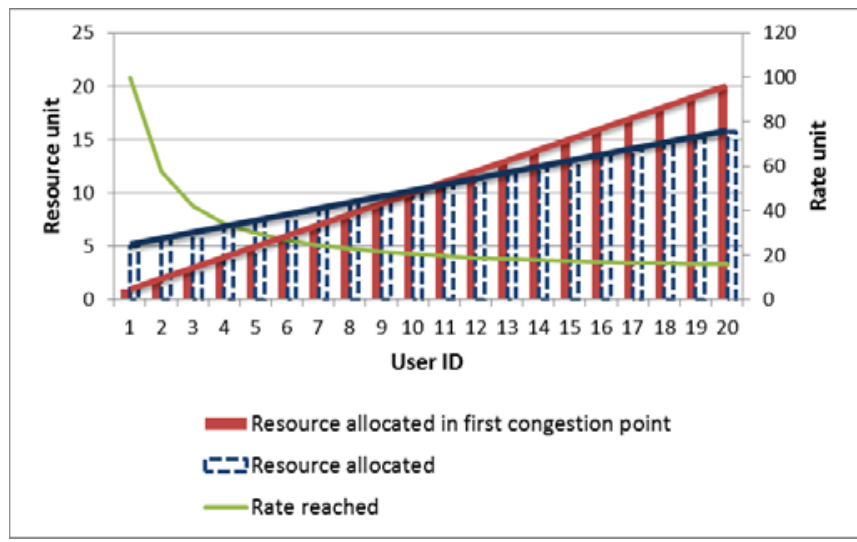

(b) Resource allocation in saturated region

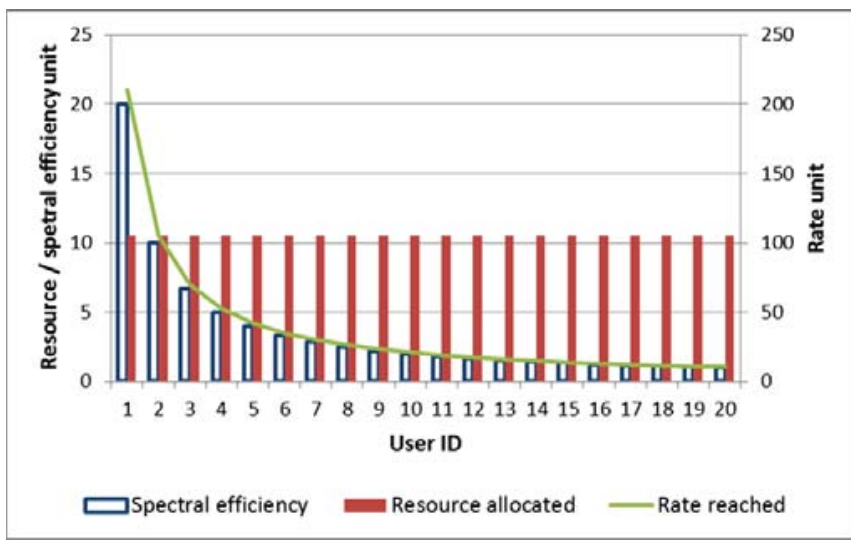

(c) Resource allocation at second saturation point

Figure 3.4: Illustration of resource allocation scheme for finite user rate demand. The numbers in the graphs have no unit, as they are just used as illustrations. The 20 users are sorted based on the spectral efficiency from 20 to 1 . In the first graph, the first saturation point is showed, when all users reached the rate demand $r_{d}$ (20 as shown by the secondary $y$ axis) and resources are mainly used by the low-spectral-efficiency users. For example, user 1 gets only 1 unit of resource while user 20 gets 20 units. When increase the rate demand $r_{d}$ to 100, user 1 get 5 units resource to reach the demand, the resource for the rest of users increase or decrease proportionally (from red bar to blue bar in (b)). The second saturation point occurs when resources are equally allocated and the users reach a rate that is proportional to their spectral efficiency. 
When $r_{d}$ increases after this point, the network enters a saturated region, i.e., all resources are used. To use the resource more efficiently, we take a certain amount of resource from the users with low spectral efficiency and give them to the users with high spectral efficiency, enabling the latter reach $r_{d}$. This process continues till all users get equal amount of resource. We call this point the second saturation point, which relates to the equal resource allocation strategy in best effort situation. This process is illustrated in Figure 3.4.

Beyond this point, to let the high spectral efficiency users reach the demand rate, more resources will be allocated to them till the extreme case where the network only serves the user with the best channel condition. In this case, the fairness deteriorates extremely. We will keep resource equally allocated after the second saturation point, and no users, even the one with the highest spectral efficiency, will achieve $r_{d}$ after this point. This region is called the over-saturated region, in which resources are equally allocated among all the uses. The rate reached for user $i$ for equal resource allocation situation is

$$
r_{i}=s_{i} \cdot \frac{W}{n_{i}},
$$

where $n_{i}$ represents the number of users in the same cell with user $i$. The two saturation points and the three regions are shown in Figure 3.5, in which the mean user rate of a typical cell is shown with respect to the rate demand $r_{d}$.

When $r_{d}$ increases, the system will reach two saturation points. The first one refers to best effort situation with equal rate allocation strategy, and the second one refers to the best effort situation with equal resource allocation strategy. The curves in Figure 3.5 show how the mean rate of the users in a cell changes with the increase of $r_{d}$, and how it matches with the rate if best effort is assumed. Before the first saturation point, i.e., the under-load region, the demands are fully satisfied, which makes the mean user rate equals to the rate demand. After that, the networks enters in the saturated region and the mean rate changes linearly because we adjust the resource proportionally from low spectral efficiency users to the high spectral efficiency users, till all resources are equally allocated. Figure 3.6 shows the effect on all the users in the system, and because different cells reach the saturation points at different time, the mean rate of all users does not change linearly. A similar resource allocation scheme considering real-time and non-real-time users is available in [35]. 


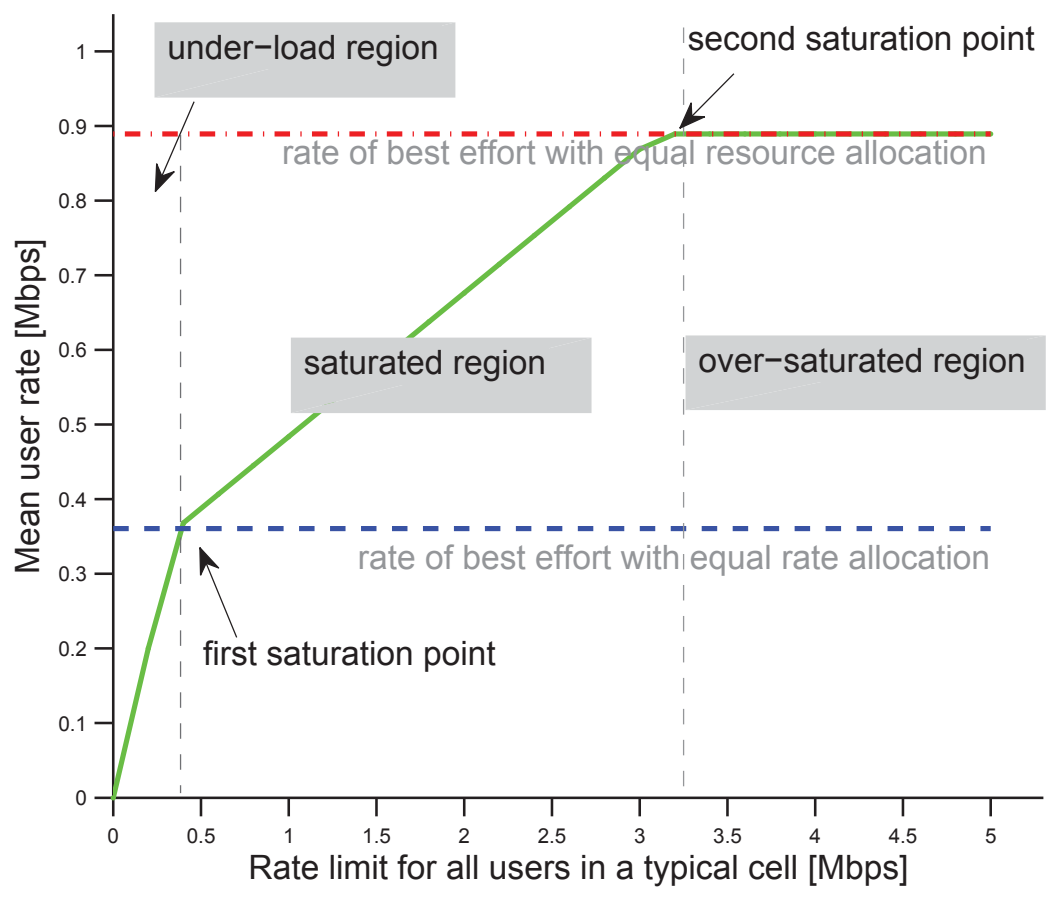

Figure 3.5: Mean user rate of the users in a typical cell with the proposed resource allocation scheme.

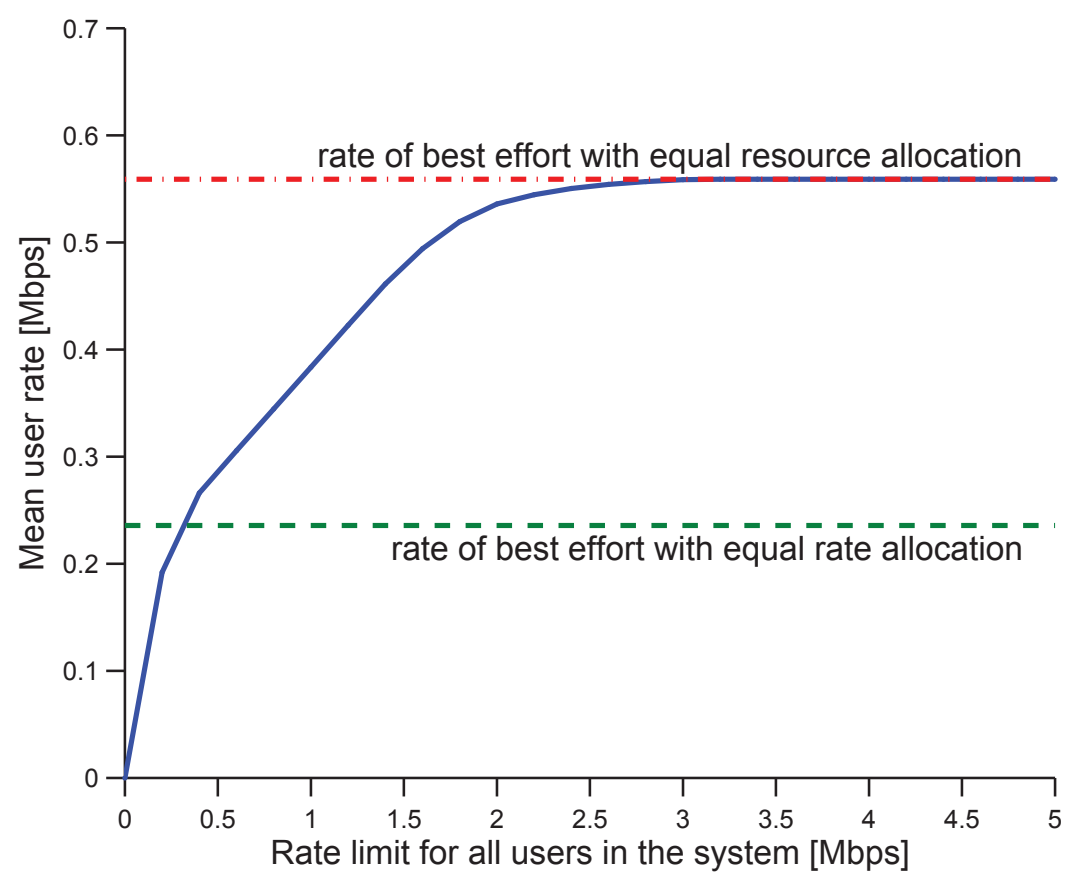

Figure 3.6: Mean user rate of the users in the system with proposed resource allocation scheme. 


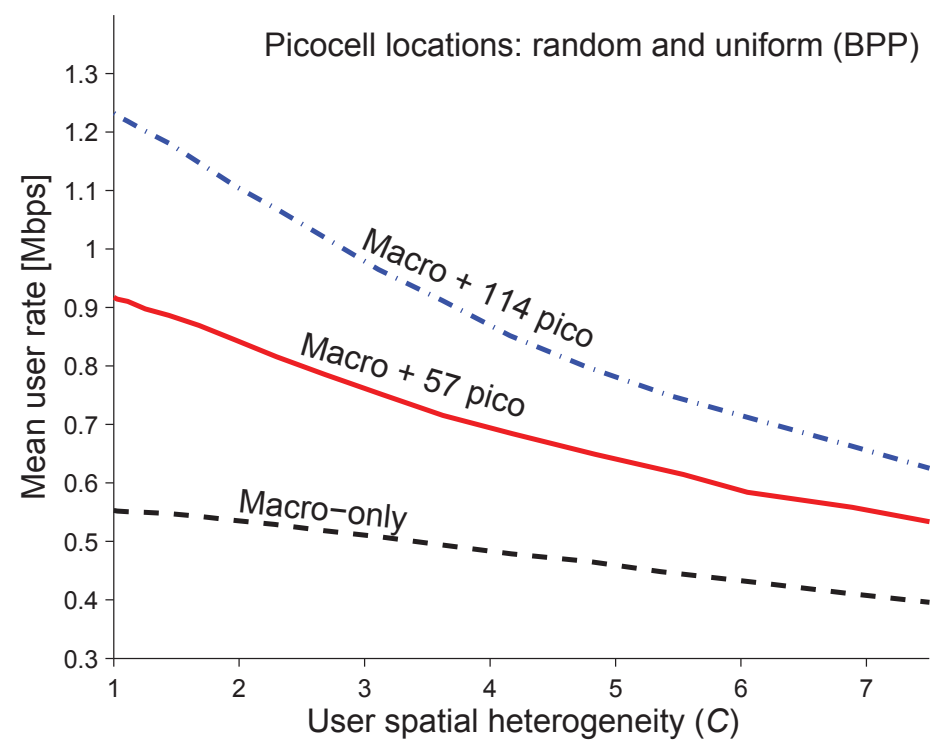

Figure 3.7: Mean user rate versus user spatial heterogeneity under different network configurations. The user distribution is a PPP when $C$ equals to 1 . Picocell locations are uniform and random based on a BPP, independent with the locations of macrocell and users.

\subsection{Performance Evaluation}

The user spatial distribution and the user traffic model are two distinguish features so far in this thesis. In this section, we first evaluate the impact of user spatial heterogeneity on macro-only networks and HetNets with different number of smallcells, under the assumption that users are served with best effort. Then the impact of finite rate demand model is evaluated next with the same settings.

\subsubsection{The Impact of User Spatial Heterogeneity}

We change the value of $\sigma$ in LGCP to get user distributions with different heterogeneities, which are measured by $C$, the normalized $\mathrm{CoV}$ of the Voronoi cell area in the Voronoi tessellations of the user points. When $C$ is equal to 1 , the user distribution forms a PPP. Performance metrics are evaluated in three scenarios: macro-only networks, pico-enhanced networks with the number of picocells equal to 57 and 114 (on average, 1 and 2 picocells per macrocell). Picocells are deployed according to 


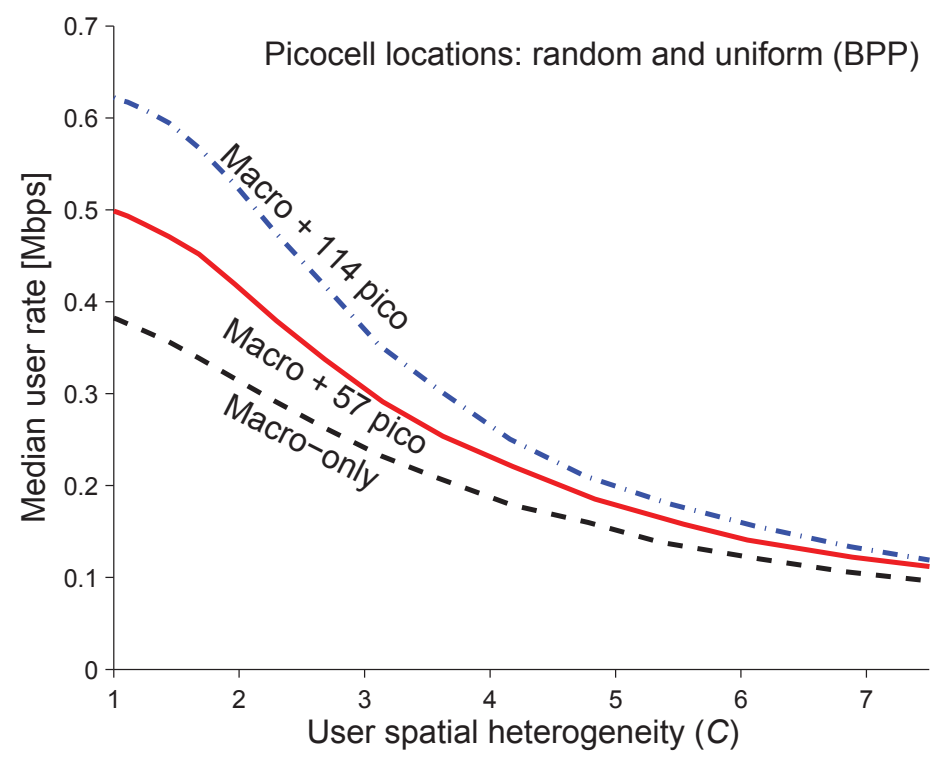

Figure 3.8: Median user rate versus user spatial heterogeneity under different network configurations.

a BPP in the macrocell covered area (as mentioned before, since the total number of picocells is fixed, the distribution of them is a conditional PPP, or equivalently, $\mathrm{BPP}[7])$.

The metrics recommended in 3GPP [18] are used in the simulation, which are the mean rate, median rate, and the $5 \%$ worst user rate. Because the overall density of users in LGCP is kept constant, the mean user rate is proportional to the sum throughout of the network, while the median user rate separates users into two halves. The $5^{\text {th }}$ percentile user rate is a metric commonly used to indicate the rates of lowSINR users, however, under non-uniform distribution of both traffic demand and capacity supply, the users that belong to this tail-rate user group may not necessarily be the low-SINR receivers, but the low share-of-resource receivers.

As we can see from Figure 3.7, Figure 3.8 and Figure 3.9, the above mentioned three metrics all deteriorate significantly with the increase in user spatial heterogeneity. This is due to the fact that when users are more spatially heterogeneous, there is a high chance that parts of the network will be highly congested, resulting in very low user rates; while the other parts of the network will be underused or even totally empty. This is true for both macro-only networks and pico-enhanced HetNets where picocells are randomly deployed. In terms of sensitivity, the $5^{\text {th }}$ percentile 


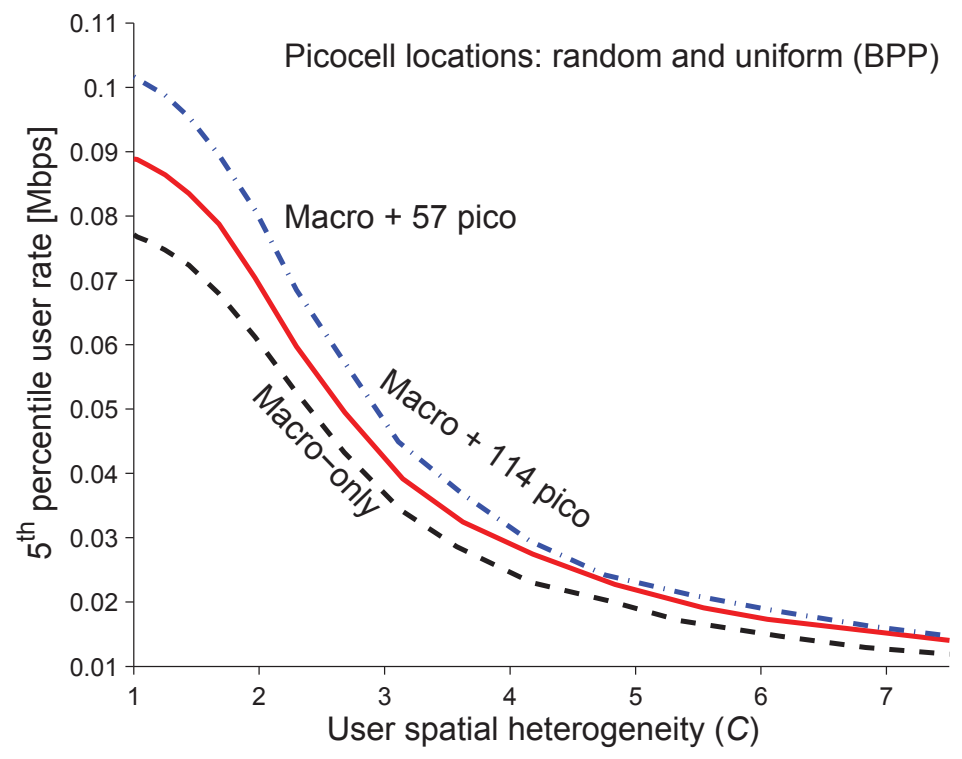

Figure 3.9: $5^{\text {th }}$ percentile of user rate versus user spatial heterogeneity under different network configurations.

user rate is the most sensitive metric (curve goes down most rapidly), as the higher user spatial heterogeneity makes the share of resource for each user more divergent, resulting in a lower $5^{\text {th }}$ percentile rate.

\subsubsection{The Impact of Finite Rate Demand}

We assume all users have the same rate limit $r_{d}$ as in Section 3.2.

Similar to Section 3.3.1, we evaluate the network performance with respect to user spatial heterogeneity under different user demand rates. The network configuration is unchanged in this evaluation. It is composed of 57 macrocells and 57 randomly distributed picocells. Figure 3.10 shows the mean user rate versus the user spatial heterogeneity under different rate limit. They all decrease monotonically with the increase in user spatial heterogeneity, and the finite user rate demand decreases the mean user rate significantly compared to the best effort model. 


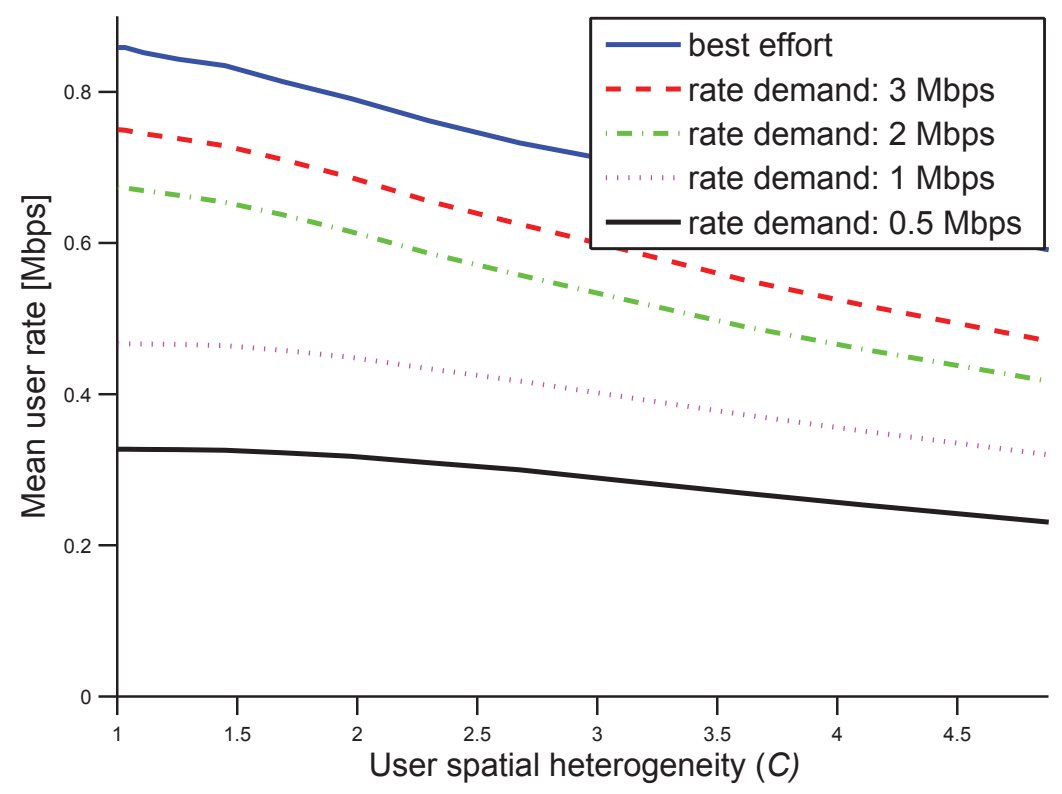

Figure 3.10: Mean user rate versus user spatial heterogeneity under different rate limit.

\subsection{Summary}

In this chapter, we introduce the guidelines of the performance evaluation from ITU and 3GPP. The simulation setup including network layout, antenna characteristics, and channel model is presented. Instead of best effort model for user traffic, we propose a finite user rate demand model, in which users have a fixed rate demand $r_{d}$. A corresponding resource allocation scheme regarding increasing $r_{d}$ is proposed. Finally, network performance is evaluated with increasing user spatial heterogeneity under different network configurations, and the results show that the network performance deteriorates significantly if user locations are uncorrelated with BS locations. The impact of finite user rate demand is also evaluated, which shows that the finite rate model decreases the mean user rate compared to the commonly used best effort model. 


\section{Chapter 4}

\section{Pushing Capacity Supply to Traffic Demand}

With a spatially non-uniform user distribution, some areas of the network may have no user or only few users, and hence the resources of the BSs in those areas are either totally wasted or underused. On the other side, the so-called hot-spot areas may be congested with users inside suffering from low rates. One solution to this problem is to deploy small-cells in the user hot-spots to offload traffic from macrocells. HetNets with small-cells overlaid on macrocells have intensely been researched in recent years, yet the distribution of small-cells is assumed to be a BPP in most of the papers. However, given an inhomogeneously distributed set of users (as proposed in this thesis), how to find the hot-spots from the user distribution to deploy smallcells is a natural, yet non-trivial, question. This is especially true for the operatorplanned picocells, which are deployed by the network operators based on the traffic distribution.

In this chapter, we first present the related work regarding the small-cell deployment (or placement) strategies, and then we propose a heuristic cluster-analysis-based small-cell deployment method. Network performance is evaluated next with comparisons between the proposed small-cell deployment method and the independent random method used in Chapter 3.

\subsection{Related Work}

The impact of placement of small-cells on downlink performance for cellular networks is evaluated in [36], in which users are deployed along the boundaries of the 
cells and the frequency reuse scheme between macrocells and microcells is studied inside. The impact of relay station placement on the network performance in the IEEE $802.16 \mathrm{j}$ network is studied in [37], in which a heuristic algorithm is proposed to determine the number and locations of relay station deployment, with a given user distribution and deployment budget. The user distribution is random or concentrated in one hotspot with a certain probability. Relay deployment in cellular networks is investigated in [38], and femto-cell self-deployment in a multi-room indoor environment is studied in [39].

The work in [40] has a similar considerations regarding small-cell deployment with the method we proposed. In this paper, a Gibbs sampling method is proposed to jointly optimize the locations of small-cell BSs in a multi-cell network with the goal of maximizing any given network utility function. Both of our methods have two considerations: (1) placing small-cells close to the high-traffic areas; and (2) avoiding co-channel interference with the macrocells. However, the traffic in this paper is not modeled by a general heterogeneous user distribution as in this thesis. Instead, it is determined by a traffic profile, in which a predefined traffic density map is used. The area of interest is a rectangular area, and is divided into a number of rectangular mini-cells. Each mini-cell has different traffic density and the small-cells are proposed to deployed in the centers of the mini-cells.

\subsection{Cluster Analysis}

The cluster analysis technique groups data into clusters such that the objects in the same cluster are more similar to each other than to those in a different cluster. This is a main task in data mining and has played an important role in a wide variety of fields, including machine learning, image analysis, and information retrieval [41]. This section uses the cluster analysis technique to find the user clusters as the potential locations for small-cells.

\subsubsection{Basic $k$-means Algorithm}

The $k$-means algorithm is one of the most popular clustering algorithms used in the cluster analysis. It is a prototype-based, partitional clustering technique that attempts to find a user-specified number of clusters $(k)$ represented by their centroids. 
The centroids are the mean of the points that belong to the cluster. The basic $k$-means algorithm [41] is described below.

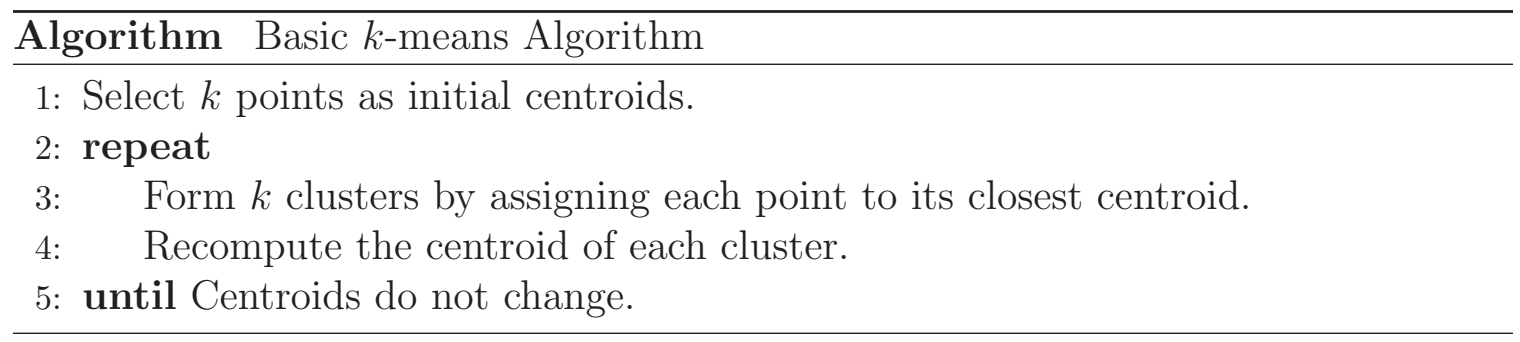

\subsubsection{Preprocessing and Postprocessing}

As we intend to use the centroids of the clusters to deploy small-cells, outlier users (isolated points) that are supposed to be served by macrocells are not taken into account. We apply preprocessing to eliminate the isolated points from affecting the locations of the centroids. A classification method of points in another densitybased clustering algorithm, DBSCAN [41], is used. All points are defined as being (1) in the interior of a dense region (i.e., a core point), (2) on the edge of a dense region (i.e., a border point), or (3) in a sparsely occupied region (i.e., a noise or background point). More precisely, a point is a core point if the number of points within a certain threshold radius of its neighborhood exceeds a certain threshold. A border point is the point that falls within the neighborhood of a core point but that is not a core point. A noise point is any point that is neither a core point nor a border point. They are illustrated in Figure 4.1. After the classification, the noise points (outlier users) are eliminated before applying the $k$-means algorithm. An example is shown in Figure 4.2.

The planned number of small-cells can be used as the value of $k$ in the $k$-means algorithm. However, since the users may not naturally be clustered into $k$ groups, the clusters that are obtained from the $k$-means algorithm may turn out to be too big for the coverage of a typical small-cell. In other words, a centroid may turn out to be in the middle of two or more natural user clusters when $k$ is small. A simple yet effective way to avoid this situation is to enlarge $k$ by splitting the clusters (by running clustering algorithm iteratively inside the cluster), a technique that is commonly used 


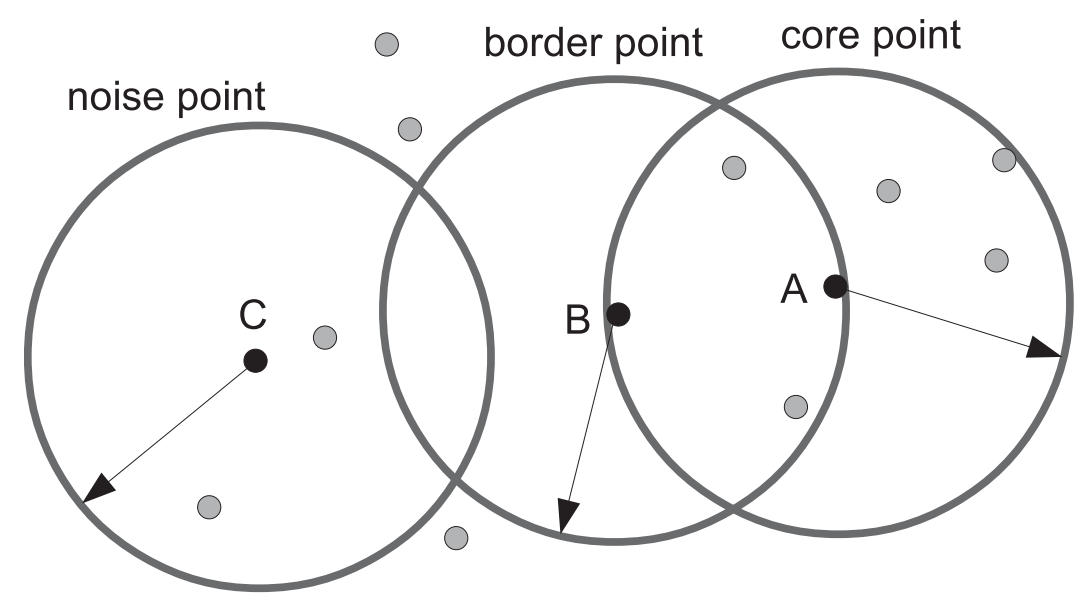

Figure 4.1: An example of core, border, and noise points. The threshold number of points is 5 in this example.

in the postprocessing for cluster analysis [41]. In our case, all clusters that have a larger radius than the typical coverage distance of the planned small-cells are split after the $k$-means clustering algorithm. After the postprocessing, $k^{\prime}$ (greater than or equal to $k$ ) clusters are obtained. An example of cluster splitting is shown in Figure 4.3 .

\subsubsection{Selection Criteria}

After postprocessing, more than $k$ clusters are obtained, potentially $k^{\prime}$ hot-spots. As only $k$ small-cells are planned, a selection criterion is needed to choose $k$ clusters from the $k^{\prime}$ clusters generated by the clustering algorithm.

A simple way is to determine the number of points $n_{i}$ in each cluster $i$, and then to choose the top $k$ out of $k^{\prime}$ clusters with respect to the number of points in them. However, when a cluster is close to a macrocell, a small-cell deployed in such a hot-spot will suffer substantial interference from the macrocell in a co-channel scenario. Even in a non-co-channel scenario, deploying small-cells close to the center of a macrocell is not as efficient as deploying them at the edge of a macrocell, as the latter improves the user spectral efficiency and provides more capacity at the same time.

In this thesis, we use the ratio of the distance between a user and a macrocell, and that between a user and a potential small-cell, as a component in the objective function to select $k$ hot-spots from $k^{\prime}$ clusters. We will also use the number of users 


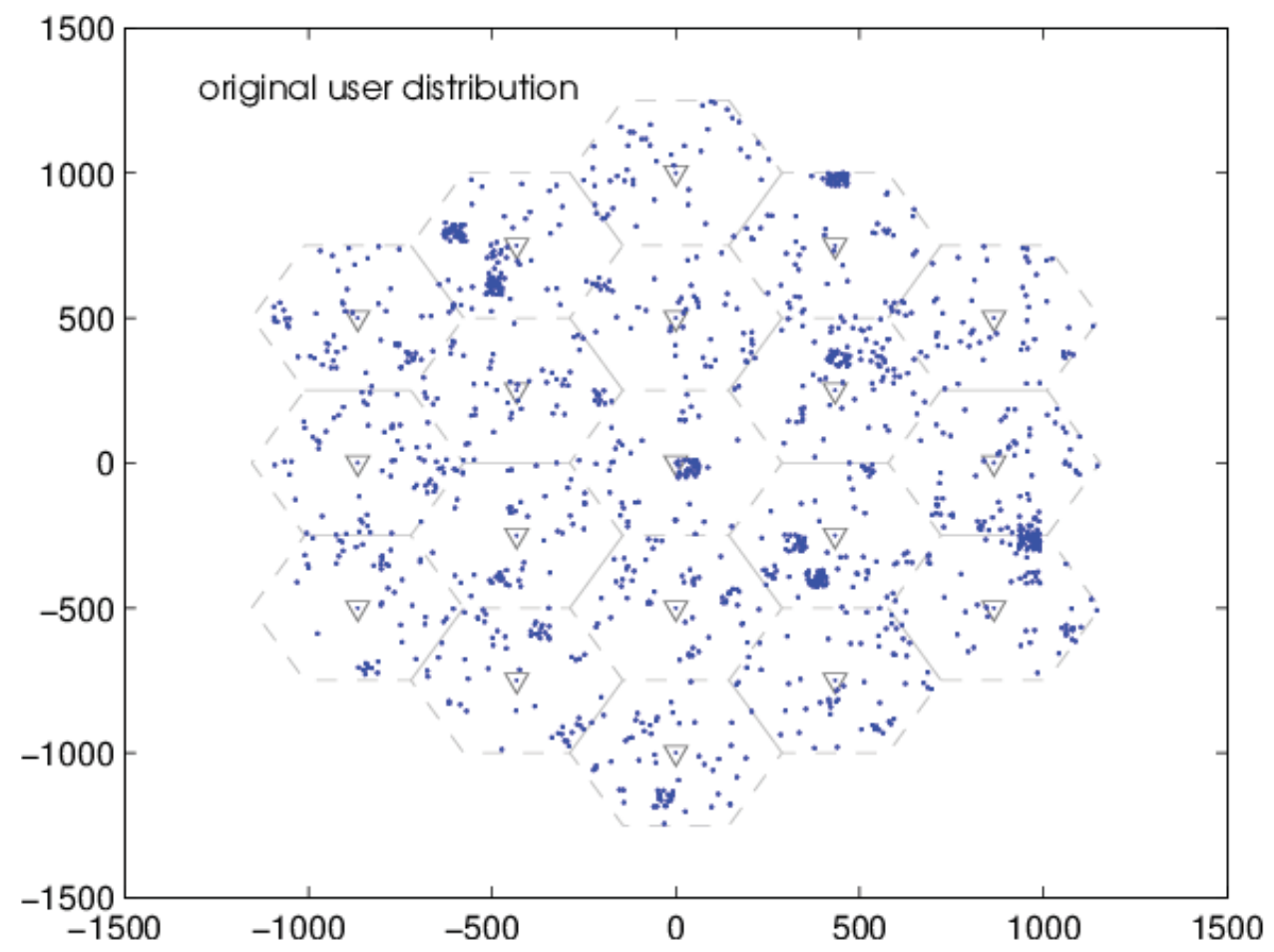

(a) Original user points

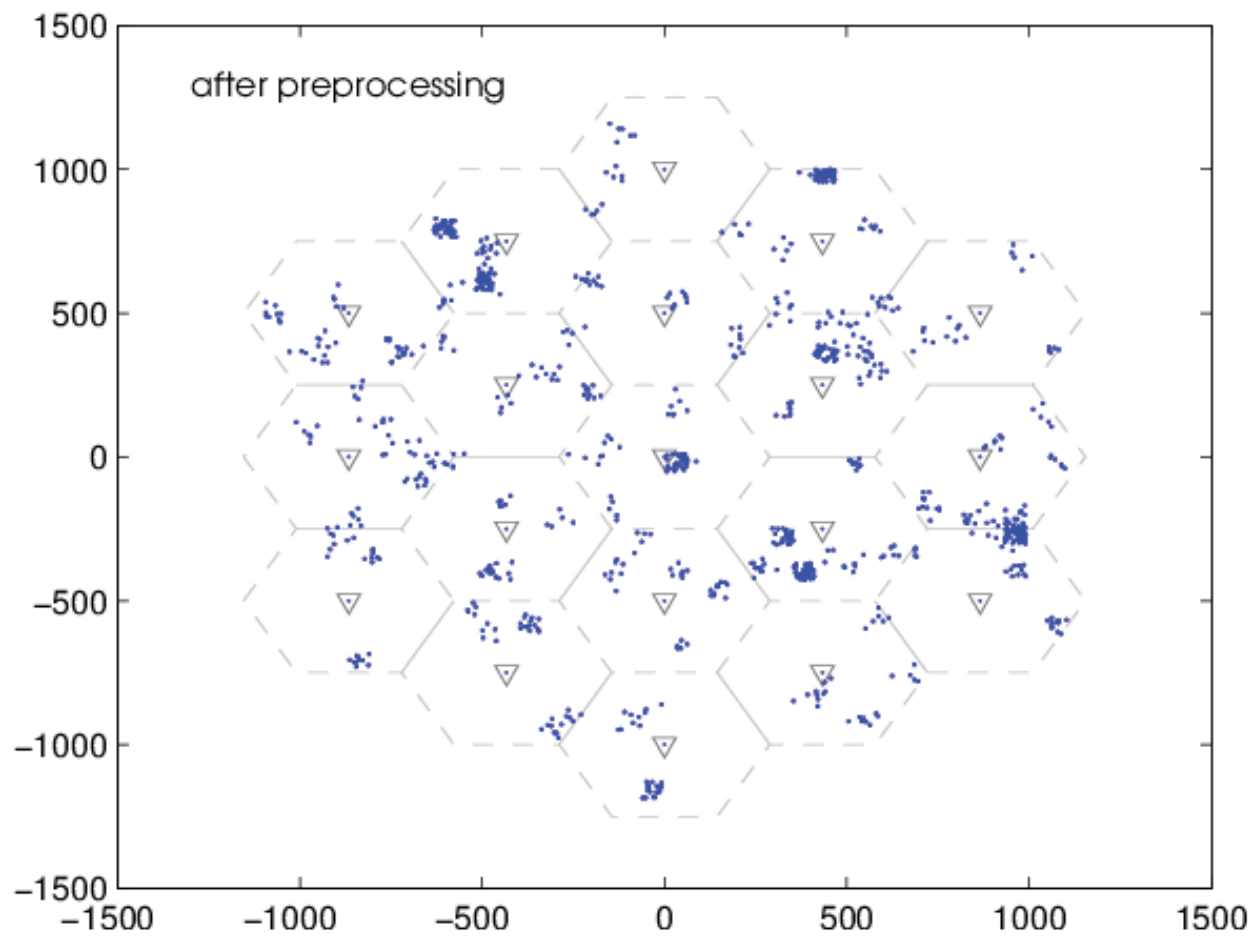

(b) User points with outlier users removed

Figure 4.2: Example of noise points elimination in preprocessing of $k$-means. 


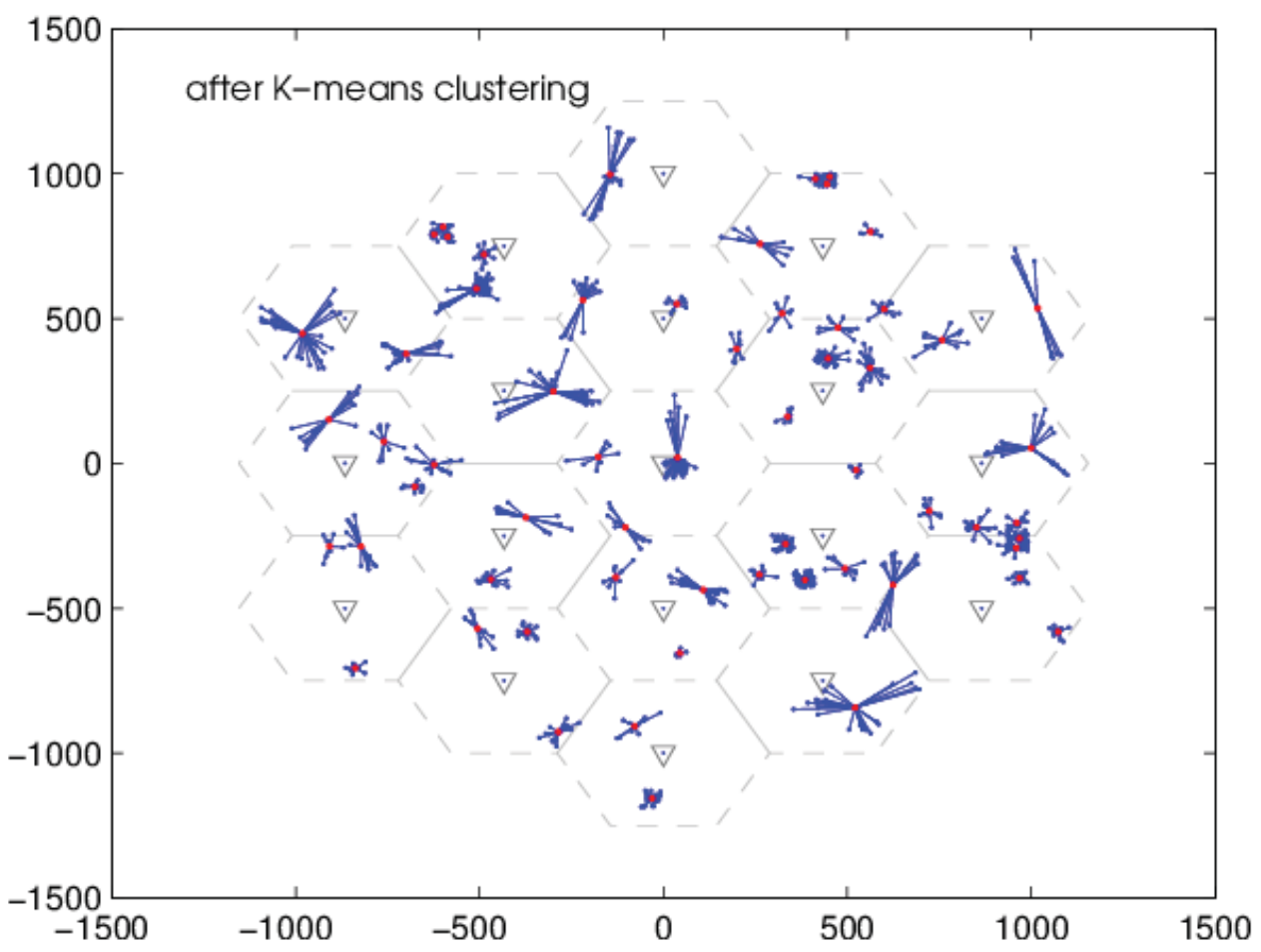

(a) After $k$-means clustering

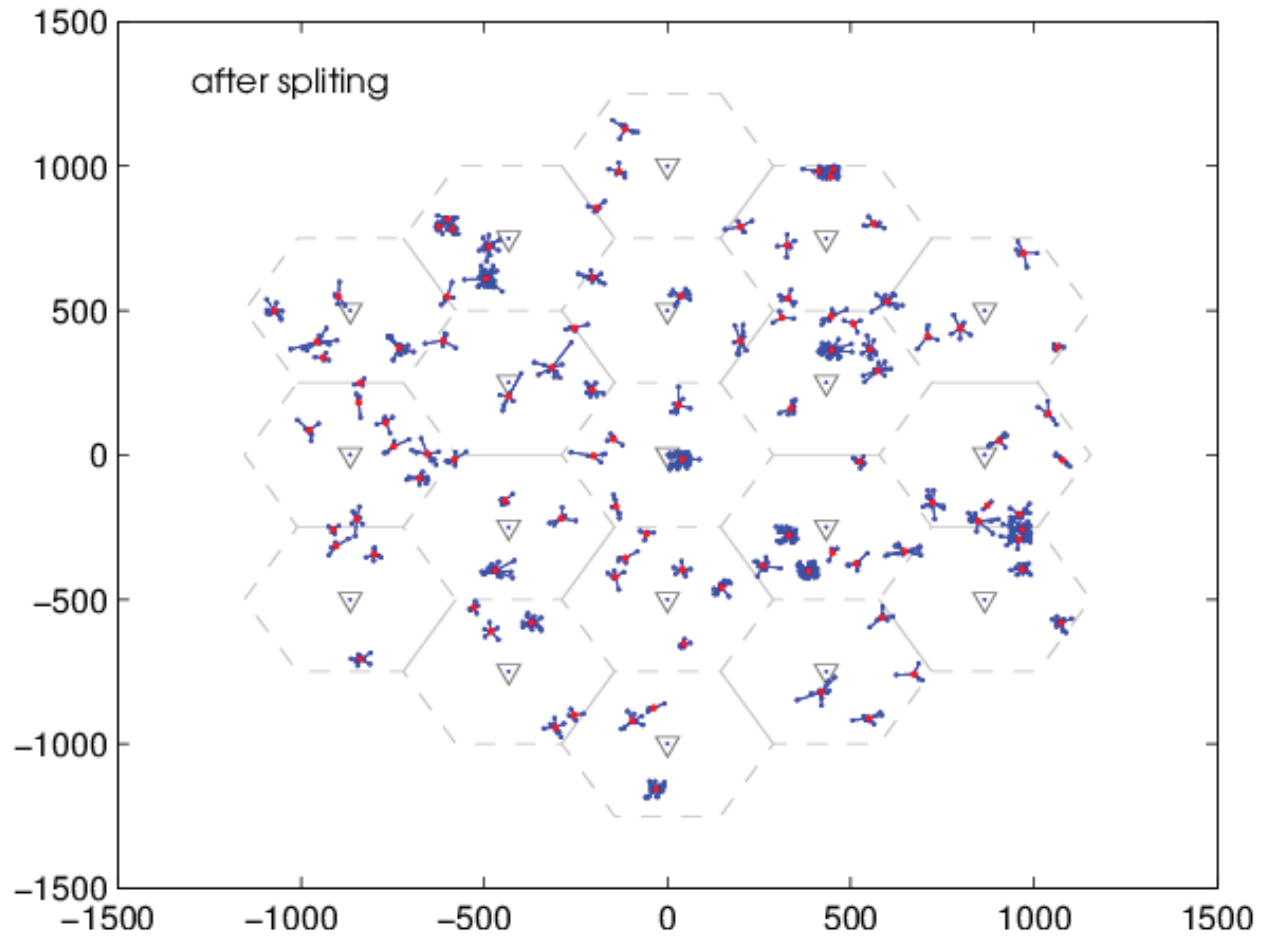

(b) After splitting

Figure 4.3: Example of $k$-means clustering and the splitting in postprocess. 
criterion as the baseline method for comparison in Section 4.3. An example of cluster selection based on the number of users in the cluster and the objective function is shown in Figure 4.4.

Suppose that there are $n_{i}$ users in a cluster $i$; for these $n_{i}$ users, $d_{j}^{(m)}$ and $d_{j}^{(s)}$ represent the distances of user $j$ to its closest macrocell, and to its cluster centroid (the location of the planned small-cell), respectively. The proposed selection criterion for cluster $i$ is formulated as

$$
U_{i}=\frac{1}{n_{i}} \sum_{j=1}^{n_{i}} \log \frac{d_{j}^{(m)}}{d_{j}^{(s)}} .
$$

This objective function is basically derived from the Shannon formula and the path loss expression. The goal is to select $k$ clusters that have maximum sum user rate, $\sum R_{j}$, which is proportional to the mean of the sum spectral efficiency $\frac{1}{n_{i}} \sum_{j} \log (1+$ $S I N R_{j}$ ) when equal resource allocation is used. In our situation, it is reasonable to ignore the background noise and assume a high signal-to-interference-ratio (SIR) as users are close to the proposed small-cells. So the objective function becomes $\frac{1}{n_{i}} \sum_{j} \log \left(S I R_{j}\right)$. Let us assume that the dominant interferer, which is from the closest macrocell, is the only interferer, and that both signal power and interference power are calculated from the a power-law based path-loss model with the same exponent. Then $\sum_{j} \log \left(S I R_{j}\right)$ becomes proportional to $\sum_{j} \log \left(d_{j}^{(m)} / d_{j}^{(s)}\right)$, the sum-logarithm of the ratio of the user to closest-macro distance to the user to planned-small-cell distance. Since this objective function is formed under several assumptions, it is rather approximate. However, this is not a concern, because this objective function is not used for evaluation; it is rather used for ranking the candidates (clusters). If interference coordination between the macrocells and the small-cells within its coverage is used, the function can be adapted to have the second nearest neighbor macrocell as the main interference source.

\subsection{Performance Evaluation}

\subsubsection{Small-cell Deployment Strategy}

This part evaluates the network performance with respect to user spatial heterogeneity under two different small-cell deployment strategies: 1) random and uniform 


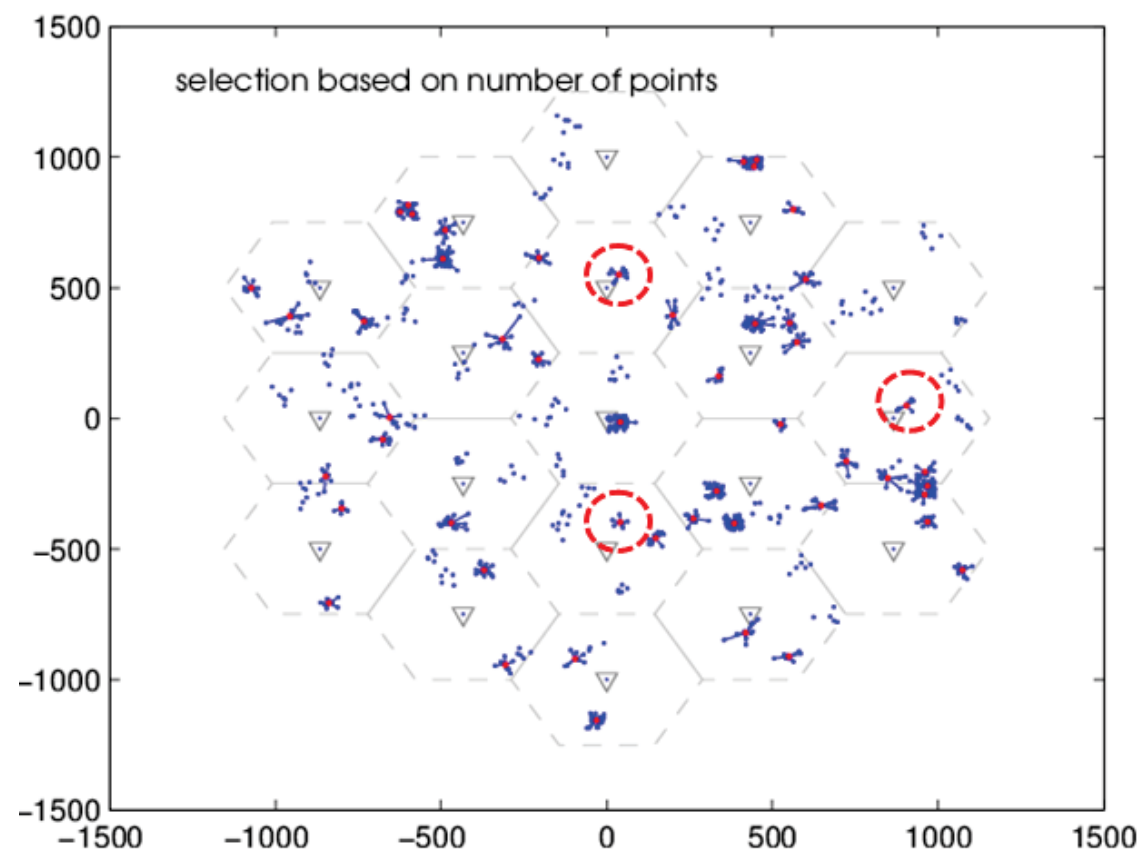

(a) Selection based on number of points in the cluster

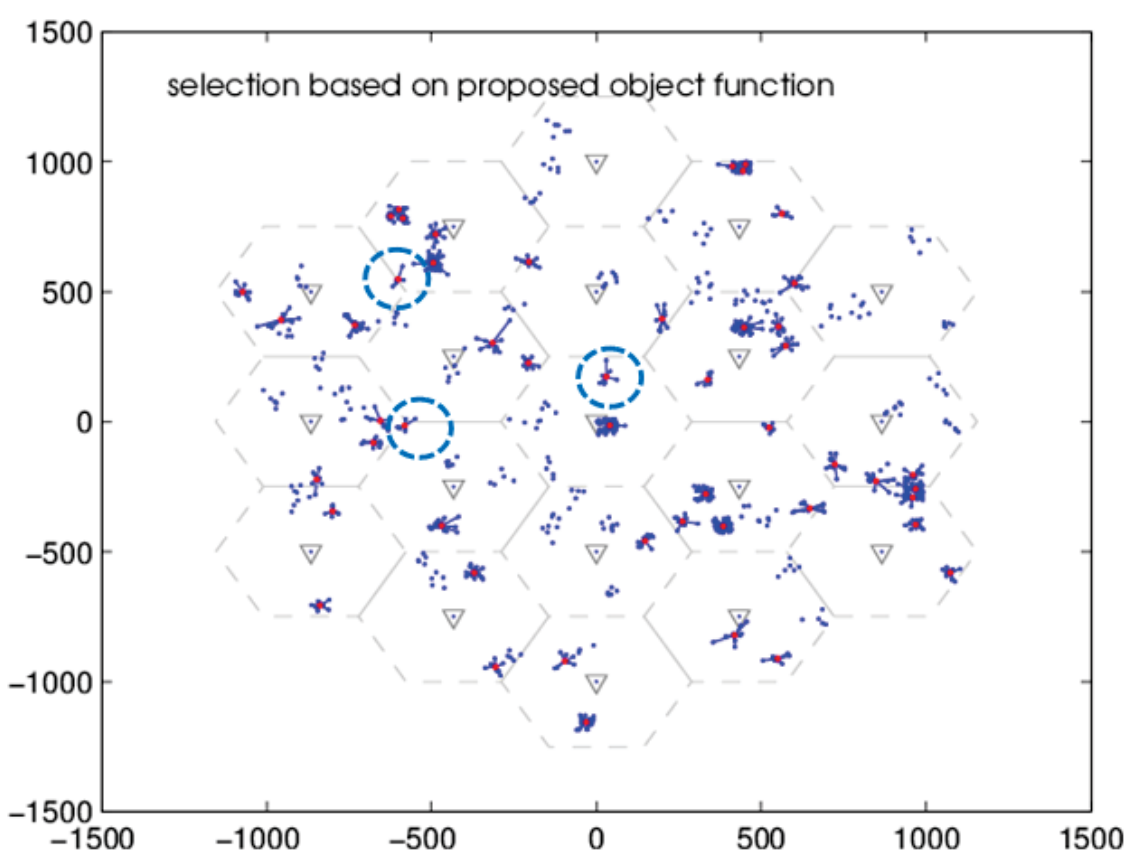

(b) Selection based on the proposed objective function

Figure 4.4: Example of different cluster selection criteria. The clusters on the top graph with red circle are the clusters that are too close to macrocells. They are not chosen in the proposed selection method shown in the graph below, instead, the clusters (blue circle) in cell edge are chosen. There is a cluster near the origin (in the center of all hexagons) which is selected to deploy a small cell near macrocell in both methods because of its high user density. 
(BPP), and 2) centroids of clusters obtained by cluster analysis. The number of picocells is kept to 57 in this section. For cluster selection, two criteria are compared: the number of users in the cluster and the proposed objective function defined in (4.1).

As we can see from Fig. 4.5, in comparison to the BPP strategy shown in the bottom curve (identical to the middle curve in Figure 3.7), the cluster center strategy with the proposed objective function discussed in Section 4.2.3 improves the mean user rate by more than 50\%. Besides, instead of decreasing monotonically with the increasing user spatial heterogeneity, the mean user rate increases first and then decreases when cluster analysis is applied for choosing picocell locations. The cluster center strategy performs better than the BPP strategy because of two reasons: 1) by bringing small-cells to the centers of the traffic demand, the load among the cells becomes more balanced; 2) the spectral efficiency is improved since the distance between the transmitters and receivers is shortened. However, a higher user spatial heterogeneity (more or larger user clusters) is beneficial to spectral efficiency improvement with appropriately deployed small-cells, but may also make the traffic load more imbalanced. When the traffic imbalance caused by user spatial heterogeneity outweighs the capacities of all the small-cells, the performance goes down. This observation gives us the insight that a certain degree of user spatial heterogeneity can be explored by correlating the locations of users and small-cells in HetNets.

\subsubsection{Fairness Index}

The widely used Jain's index is evaluated to quantify the rate fairness among all the users. Note that the fairness evaluated here is different from the commonly used measure that indicates whether users or applications are receiving a fair share of the system resources. In this section, we use Jain's index to measure the fairness of all users in the system in terms of the rate. It is defined as

$$
\mathcal{J}\left(x_{1}, x_{2}, \ldots, x_{n}\right)=\frac{\left(\sum_{i=1}^{n} x_{i}\right)^{2}}{n \cdot \sum_{i=1}^{n} x_{i}^{2}}
$$

where $n$ denotes the number of users and $x_{i}$ denotes the user rate for user $i$. Figure 4.6 shows the fairness index versus user spatial heterogeneity under different network configurations. 


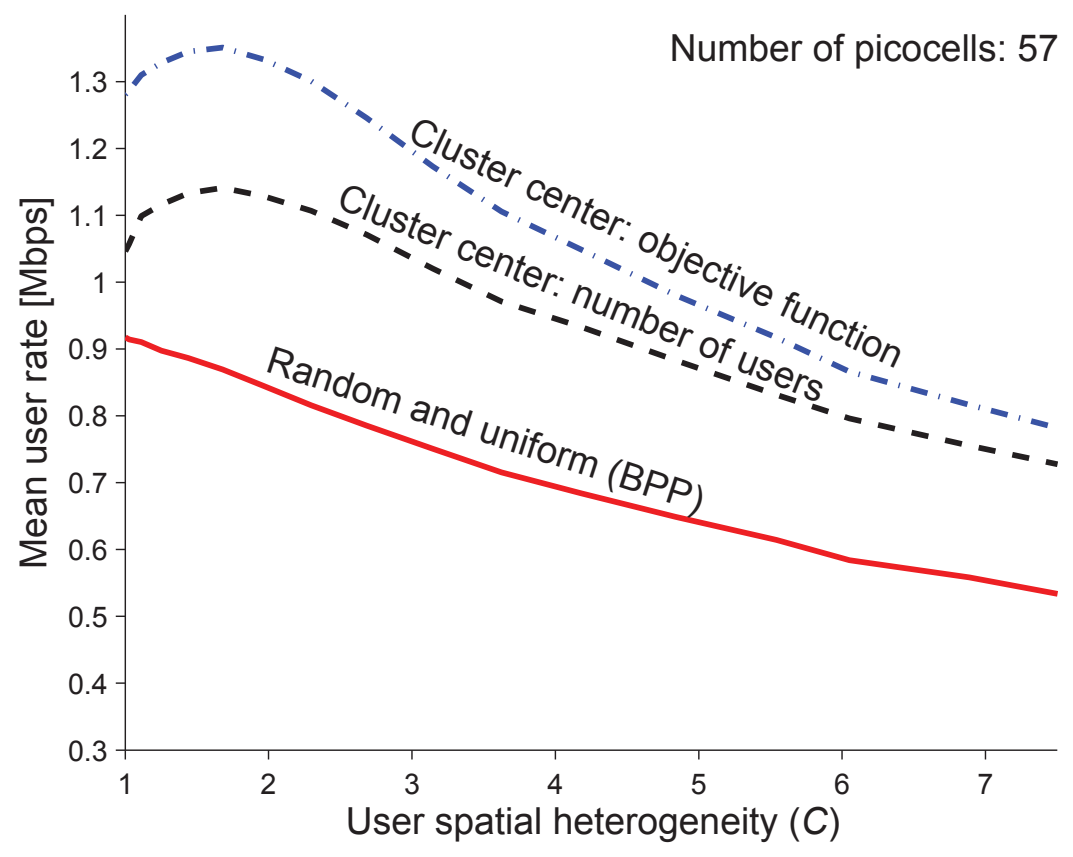

Figure 4.5: Mean user rate versus user spatial heterogeneity with different small-cell deployment strategies and different cluster selection methods.

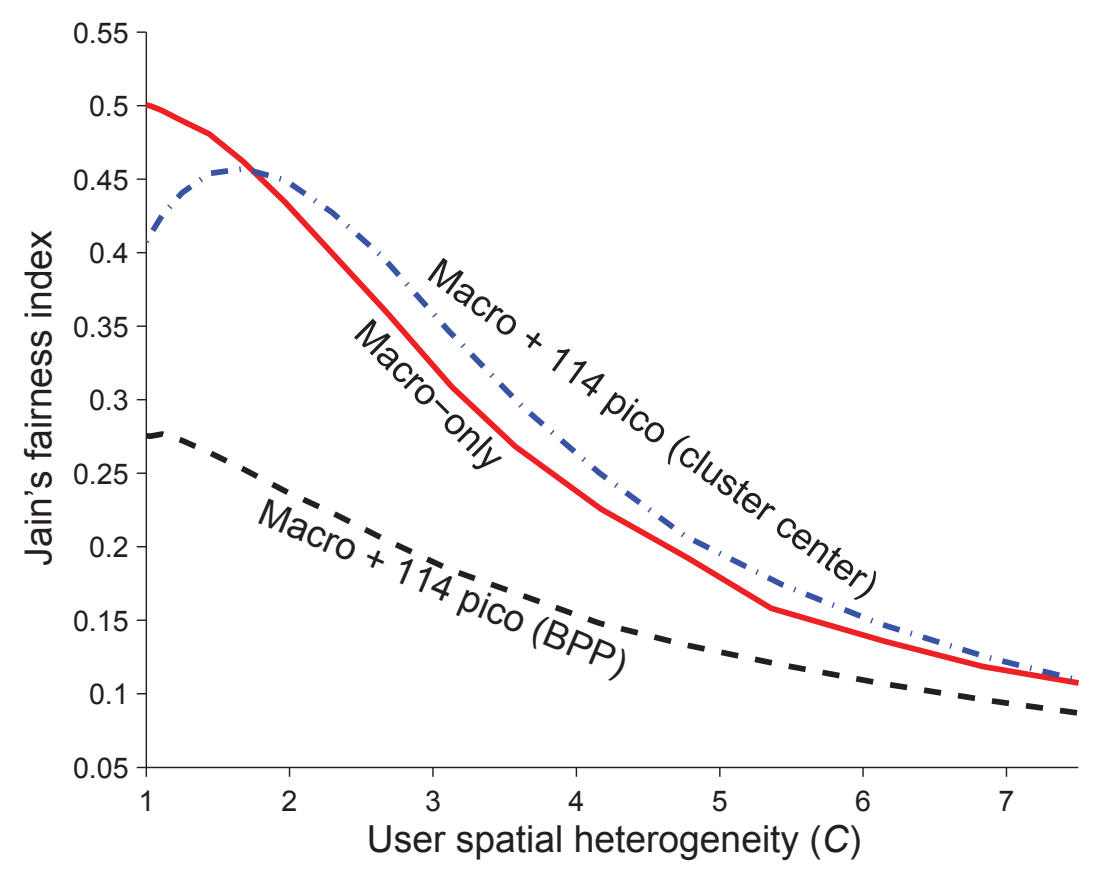

Figure 4.6: Jain's fairness index of user rate versus user spatial heterogeneity under different network configurations. 
The fairness index goes down monotonically when users are more heterogeneously distributed in the marco-only networks, and in HetNets where small-cells are randomly and uniformly deployed (BPP); the Jain's index is even worse in the latter. The downwards trend is because of the fact that the increasing spatial heterogeneity causes more resource imbalance. The performance gap between the macro-only networks and HetNets is as a result of the randomly located picocells which serve only a small portion of the users, letting them reach high rates (which in turn worsens the fairness index). Similar to Figure 4.5, when picocells are deployed based on the clustering algorithm introduced in Section 4.2, the fairness index rises first and then goes down with the increasing user spatial heterogeneity.

\subsection{Summary}

This section proposes the first approach to combat the traffic imbalance problem in HetHet cellular networks (HetHetNets), which is to push capacity supply to the traffic demand. By using the cluster analysis technique, the user cluster centers are obtained from the non-uniform user points as the potential small-cell locations. A selection method that considers the relative distance between user to macrocells and user to potential small-cells is introduced to choose clusters to deploy small-cells. It is observed that if small-cells are deployed based on the user spatial distributions, the performance of HetNets can benefit from the increase in user spatial heterogeneity up to a certain degree. 


\section{Chapter 5}

\section{Pushing Traffic Demand to Capacity Supply}

Other than pushing capacity supply to traffic demand as in Chapter 4, a dual approach for load balancing with traffic shaping by user-in-loop (UIL) is introduced in this chapter. We show that the spectral efficiency for the users and the load balancing for the system can be improved at the same time, resulting in significant network performance enhancement.

The rest of this chapter is organized as follows. First the related work is investigated in Section 5.1, including load-aware cell association and UIL concept.

In Section 5.2, the system model for spatial traffic shaping is introduced. The diagram inside explains how the user is incorporated as part of a closed-loop control system. A machine learning functionality is also included in the system for the UIL controller to better predict the user behavior, which provides more relevant and accurate suggestions.

In Section 5.3, a scenario model with two traffic classes is proposed, guaranteed bit rate $(\mathrm{GBR})$ and best effort $(\mathrm{BE})$. A resource allocation scheme to guarantee the priority of the GBR users over the BE users is also provided. Besides the traffic classes, the spatial distribution of users is also non-uniform, modeled by a log Gaussian Cox process introduced in Chapter 2.

In Section 5.4, the utility function for the UIL controller is introduced. Based on the nature of the system, a sequential greedy method is used to find a suitable location suggestion for each user, and the associated cell is generated automatically within the algorithm.

A load-aware cell association approach for load balancing without traffic shaping 
is introduced in Section 5.5. By using the same user model and resource allocation policy as the UIL approach, it is evaluated in comparison with the performance of the UIL approach in the numerical analysis part, Section 5.6.

\subsection{Related Work}

In HetNets, due to the disparities between macrocell BSs and small-cell BSs in terms of transmit power, antenna gain, and antenna height, the coverage sizes of these two types of BSs are massively disparate. As such, the conventional max-SINR (associate the user to the cell whose SINR is maximum) cell association strategy results in significant traffic imbalance in HetNets, a major source of performance degradation [42].

There are two possible approaches to push traffic demand to capacity supply in load balancing: (1) users are stationary but are associated with cells by taking load into consideration; and (2) users are a part of control loop and can be encouraged to relocate based on the load of the cells. The first approach relates to load-aware cell association, and the second spatial traffic shaping method is the approach we propose in this chapter.

Recently, load-aware cell association has been considered extensively in the literature as an approach to load balancing. In this approach, users are assumed to be stationary, and the association relation is the result of an optimization problem. The objective of the optimization problem is usually the sum of the utility function of all the users, and the utility function is the function of user rate in most cases, e.g., the logarithm of user rate in [43] [44] [45]. This approach is further introduced in Section 5.5 .

The UIL concept [46-54] aims at controlling the user ("layer-8") behavior in a wireless system to achieve a better performance of both the user and the network by convincing the users to move from one location to a better one or to avoid traffic congestion by postponing session traffic out of the busy hours. Based on the impact dimension, the approach is called spatial or temporal UIL control (this work only involves spatial UIL). In both cases, the user is within, as part of, a closed-loop control system.

In [47], the authors show that substantial cell spectral efficiency gain is obtained with the use of UIL. In [51], the economic aspect of the UIL concept is investigated 
in order to find relevant business cases for the operators and the wireless subscribers. The proposed temporal UIL in [50] resolves congestion situations in the busy hour and manages to let users reduce their rate of video traffic and data traffic (downloads) because of the increased dynamic price. The approach in [50] is comparable to the intention of the smart grid, with the additional feature of service classes. In [48], the question about what incentives will lead to what user reaction is answered based on survey results. Thus we are able to quantitatively describe the user behavior in a system theoretic framework.

Under the concept of the UIL, this thesis goes further with a utility function that incorporates cell-level load factor, potential SINR and the user moving probability, which leads to a novel spatial traffic shaping approach to load balancing.

\subsection{System Model for Spatial Traffic Shaping}

Under the paradigm of UIL, users are suggested opportunistically to move to a new location according to operator recommendation and incentive displayed on the user terminal. Users can choose to or not to comply with the suggestion for better service and better rewards (depending on the content of the incentive). In this thesis, the new location is the place where the user will receive higher SINR and/or more resource share, and the user is willing to move to. It is given by the UIL controller (see Figure 5.1) based on the utility function, which considers SINR maps of all cells (the potential spectral efficiencies of the new locations with different cells), the load factor of each cell and the probability of moving to different locations (related to application class, user behavior and so on). Load balancing is achieved by the spatial movement of users which comply with the suggestions. By shaping the traffic spatial distribution, the traffic demand is controlled to meet the capacity supply better, the distribution of which usually stays unchanged once the placement of BSs has been completed.

The UIL system theoretic model for traffic shaping is shown in Figure 5.1. Instead of assuming users being a traffic generator and consumer as a black box only, the UIL framework allows a control input into the user block, on which the user receives suggestions and incentives (i.e., progressive tariffs, reward points, higher access rates, or even environmental indicators) in order to convince him to move to a new location. The control information (CI) is sent from the UIL controller in form of suggestions 


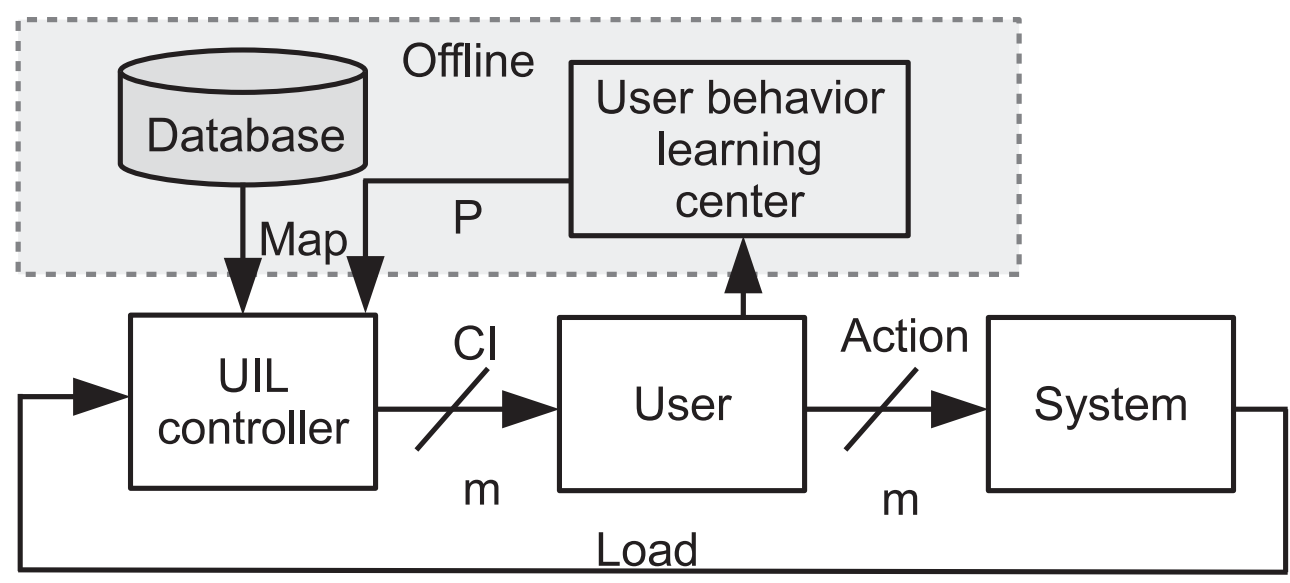

Figure 5.1: The UIL system theoretic model diagram. The UIL system theoretic model is a closed loop with the user included in the system to control. The user's output is the action of complying or not with the suggestions. As we have multiple users in a cell, the arcs between controller, user, and the system are vectorized for $m$ users.

on the user terminal graphical interface (e.g., a map with directions). The suggestion is opportunistic (not mandatory) and users can choose to comply or not. The action of the users (cooperate or not) is then returned to the cellular network system. The suggestions are the main output of the UIL controller based on the utility function discussed in Section 5.4, and the incentive is set by the operator considering factors like the current tariffs, the marketing policy, the network congestion degree, and so on.

The input of the UIL controller includes three components which are: 1) the map information from the database, 2) the user probability of moving, $P$, from the user behavior learning center, and 3) the cell load information, $L$, from the cellular network system. The map information includes city map that facilitates the movement of users and the spectral efficiency map used to calculate the utility function. The spectral efficiency map is generated from the network with the measurement from all the user terminals being accumulated and statistically averaged. It is relatively constant and only needs to be updated when the network configuration or the city landscape changes.

$P$ is the output of the user behavior learning center, which learns the user behavior under different circumstances. For example, the probability of user $u$ with quality of 
service (QoS) $q$ to move distance $d$ with incentive $i$ within the user's context $c$ can be formulated as $P_{u}(d, q, i, c)$. A QoS level may be real-time, non-real-time, or extensions based on contract, e.g., gold, silver, or bronze. The incentive may take different forms: financial bonus, penalty, extra capacity, or even environmental indicators. The user's context $c$ can be various. The user may be known to be immobile, for example in a stadium. The user may be known to pay all penalties and discard all incentives. The user options may be set to suppress all UIL suggestions. The user and application may be postpone intolerant as it might be the case with a business phone call. The connectivity of the user to other peers nearby may be part of the context as well. Other human, social or technical aspects may also be part of the context. The initial $P$ could be quite general. For example, it could be the function of distance $d$ and QoS $q$ alone as we used it in the numerical analysis in Section 5.3.3. With the evolution of machine learning, more specific output could be possible, e.g., the probability of one specific user at a specific location to move to another specific location at a specific time.

While the map information and the value of $P$ are being provided from an offline database, the cell load $L$ is included in the closed loop and is updated simultaneously when a new user session arrives to or departs from the system.

\subsection{User Model}

\subsubsection{User Traffic Classes}

Unlike most papers in the literature which assume one class of users only (best effort or best effort with minimum rate), we model users with two different service classes: guaranteed bit rate (GBR) and best effort (BE). An example of these two service types could be real-time video application and FTP/HTTP download, respectively. The GBR users have a guaranteed rate and a higher priority, while BE users share all the resources in the cell that are not used by GBR users. In this work, we assume that GBR users are guaranteed a fixed rate $r$ when the service is available or receive no service at all (i.e., outage) if the resources are not enough for the user to reach the guaranteed rate $r$. 


\subsubsection{Resource Allocation}

We denote by $\mathcal{C}$ the set of all cells (including macrocells and small-cells). The total resources of a cell $j \in \mathcal{C}$, denoted by $W_{j}$, are shared among all the users associated with it. The resources can be time or frequency slots, or both. In our model, GBR users have higher priority and are allocated the exact amount of resources they need. For a GBR user $i$ with spectral efficiency $s_{i j}$ from cell $j$, the amount of resources needed from cell $j$ to reach the guaranteed rate $r$ is

$$
w_{i j}=\frac{r}{s_{i j}}
$$

$s_{i j}$ is derived from the Shannon formula,

$$
s_{i j}=\log \left(1+\frac{P_{j} g_{i j}}{\sum_{h \in C, h \neq j} P_{h} g_{i h}+\sigma^{2}}\right),
$$

where $P_{j}$ is the transmit power of cell $j, g_{i j}$ denotes the channel gain between user $i$ and cell $j$. The channel gain includes antenna gain, path loss and shadowing. Fast fading is not considered here as an averaged SINR is assumed over the session length. $\sigma^{2}$ represents the noise power in SINR.

When a new GBR user $i$ arrives to cell $j$, the user will be allocated the exact amount of resources $w_{i j}$ that is needed if

$$
W_{j}-\sum_{i^{\prime} \in \mathcal{U}^{g}(i)} a_{i^{\prime} j} w_{i^{\prime} j}>w_{i j}
$$

where $W_{j}$ is the total resources of the cell $j . a_{i^{\prime} j}$ is the association indicator (i.e., $a_{i^{\prime} j}=1$, if user $i^{\prime}$ is associated with cell $j, a_{i^{\prime} j}=0$ otherwise) and $\mathcal{U}^{g}(i)$ is the set of the existing GBR users at the moment when user $i$ arrives to the system. If the Inequality (5.3) does not hold, this GBR user will be blocked, which results in the outage of this GBR user.

The BE users equally share the remaining resources that are not used by the GBR users. For example, when a new BE user $k$ arrives to the system, the resources will be reallocated to these $n_{j}^{b}(k)+1$ users, where $n_{j}^{b}(k)$ is the number of the existing BE users associated with cell $j$ at the moment when user $k$ arrives. The amount of 


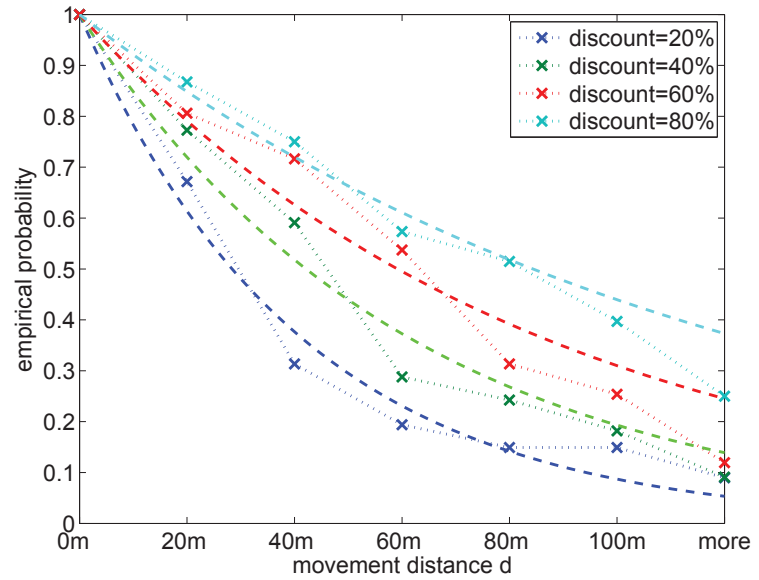

(a) Incentive as discount if user moves

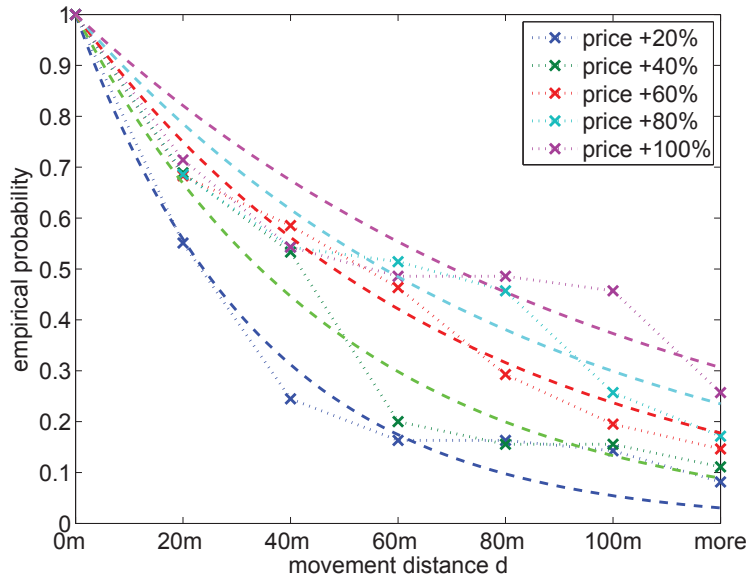

(b) Incentive as penalty surcharge if user stays

Figure 5.2: Empirical CCDF and exponential fit for data service and suggested UIL movement with a discount and penalty incentive (fit Equations (5.5) and (5.7), respectively) [48].

resources allocated to user $k$ is

$$
w_{k j}=\frac{W_{j}-\sum_{i \in \mathcal{U}^{g}(k)} a_{i j} w_{i j}}{n_{j}^{b}(k)+1} .
$$

Because of the outage mechanism of the GBR users, $w_{k j}$ is always positive, which means each BE user will be served no matter how little resources are allocated. Minimum rate demand for BE users can be added to the model, which will result in possible outage for BE users as well. Note that the arrival of a GBR user $i$ to cell $j$ also triggers the reallocation of resources for all $n_{j}^{b}(i)$ BE users in the same cell.

\subsubsection{Probability of Moving: the $\mathrm{P}$ function}

In this thesis, we construct the $\mathrm{P}$ function from $P_{u}(d, q, i)$, i.e., the function of moving distance, QoS, and incentive, without considering $c$, the user's context as introduced in Section 5.2. We adopt the data from [48], in which user behavior is modeled based on survey results. Three types of services (QoS) were included in the questionnaire: data, video, and voice. We take the results of data and video as the behavior model for our BE and GBR users, respectively.

The spatial UIL motivated relocation for data service of $d$ meters is shown as 
complementary cumulative distribution function (CCDF) in Figure 5.2, first with a discount incentive $\delta$ and second with a surcharge penalty $\pi$. $i$ is the corresponding integer index. The results show a clear control ratio. The numeric fits for the data service (BE users) are as follows [48]:

$$
\begin{gathered}
\delta_{i=1}=-20 \% \Rightarrow p=e^{-0.0244 \cdot d} \\
\delta_{i=2}=-40 \% \Rightarrow p=e^{-0.0164 \cdot d} \\
\delta_{i=3}=-60 \% \Rightarrow p=e^{-0.0117 \cdot d} \\
\delta_{i=4}=-80 \% \Rightarrow p=e^{-0.0082 \cdot d} \\
p=e^{(-0.0285+0.0053 \cdot i) \cdot d}=e^{(-0.0285-0.0265 \cdot \delta) \cdot d} \\
\pi_{i=1}=+020 \% \Rightarrow p=e^{-0.0291 \cdot d} \\
\pi_{i=2}=+040 \% \Rightarrow p=e^{-0.0202 \cdot d} \\
\pi_{i=3}=+060 \% \Rightarrow p=e^{-0.0144 \cdot d} \\
\pi_{i=4}=+080 \% \Rightarrow p=e^{-0.0121 \cdot d} \\
\pi_{i=5}=+100 \% \Rightarrow p=e^{-0.0099 \cdot d} \\
p=e^{(-0.0311+0.0047 \cdot i) \cdot d}=e^{(-0.0311-0.0235 \cdot \pi) \cdot d}
\end{gathered}
$$

The spatial UIL motivated relocation for video service of $d$ meters is shown as complementary cumulative distribution function (CCDF) in Figure 5.3, first with a discount incentive $\delta$ and second with a surcharge penalty $\pi$. The numeric fits for the video service (GBR users) are as follows [48]:

$$
\begin{gathered}
\delta_{i=1}=-20 \% \Rightarrow p=e^{-0.0276 \cdot d} \\
\delta_{i=2}=-40 \% \Rightarrow p=e^{-0.0192 \cdot d} \\
\delta_{i=3}=-60 \% \Rightarrow p=e^{-0.0127 \cdot d} \\
\delta_{i=4}=-80 \% \Rightarrow p=e^{-0.0090 \cdot d} \\
p=e^{(-0.0327+0.0062 \cdot i) \cdot d}=e^{(-0.0327-0.0310 \cdot \delta) \cdot d}
\end{gathered}
$$




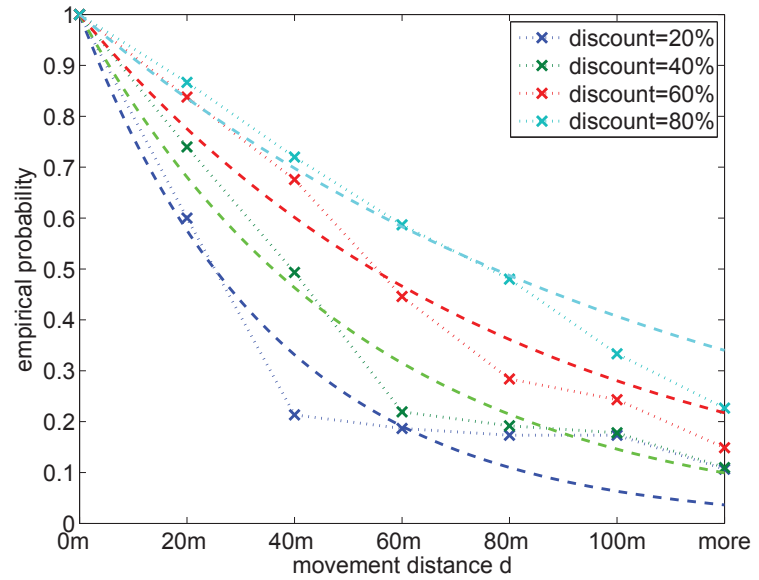

(a) Incentive as discount if user moves

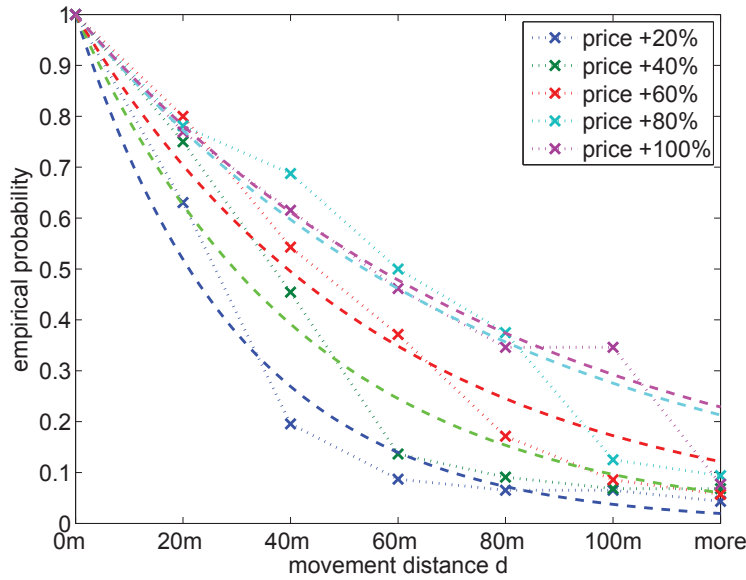

(b) Incentive as penalty surcharge if users stays

Figure 5.3: Empirical CCDF and exponential fit for video service and suggested UIL movement with a discount and penalty surcharge incentive (fit Equations (5.9) and 5.11, respectively) [48].

$$
\begin{aligned}
\pi_{i=1} & =+020 \% \Rightarrow p=e^{-0.0328 \cdot d} \\
\pi_{i=2}=+040 \% & \Rightarrow p=e^{-0.0234 \cdot d} \\
\pi_{i=3}=+060 \% & \Rightarrow p=e^{-0.0176 \cdot d} \\
\pi_{i=4}=+080 \% & \Rightarrow p=e^{-0.0129 \cdot d} \\
\pi_{i=5}=+100 \% & \Rightarrow p=e^{-0.0123 \cdot d} \\
p=e^{(-0.0352+0.0051 \cdot i) \cdot d} & =e^{(-0.0352-0.0255 \cdot \pi) \cdot d}
\end{aligned}
$$

More information can be found in [48]. All the results confirm the general intuitive trends:

- The acceptance $p$ drops with effort (distance $d$ in spatial domain as the case in this thesis).

- A stronger incentive is followed with more acceptance.

- A more forceful penalty also leads to more obedience.

\subsection{Proposed UIL Load Balancing Approach}

The UIL controller outputs the control information with the suggestion of the potential location based on the maximization of a utility function. The objective 
utility function combines three factors: the SINR value of different locations from each cell, the load of each cell, and the probability of moving from the current location to the potential locations.

Note that the utility function formulated in this section is just one way to combine all the three factors that are considered in this thesis. In our formulation, all the three factors are treated equally, i.e., they are of the same order in the function. Other formulations are also possible.

\subsubsection{Utility Function of GBR Users}

The utility function of a GBR user $i$ with cell $j$ is formulated as

$$
U_{i j}(x, y)=p_{i}(x, y) \cdot s_{j}(x, y) \cdot\left(1-\rho_{j}^{b}(i)\right)
$$

where $p_{i}(x, y)$ is the probability of user $i$ moving from his current location to the new location with coordinates $(x, y), s_{j}(x, y)$ is the spectral efficiency map of cell $j$, and $\rho_{j}^{b}(i) \in[0,1]$ is the load factor of cell $j$ for the existing GBR users at the moment when user $i$ arrives to the system. $\rho_{j}^{b}(i)$ is defined as

$$
\rho_{j}^{b}(i)=\frac{\sum_{i^{\prime} \in \mathcal{U}^{g}(i)} a_{i^{\prime} j} w_{i^{\prime} j}}{W_{j}},
$$

where $a_{i^{\prime} j}$ is a known matrix indicating the association relation of all the GBR users at the moment when user $i$ arrives to the system, and is updated each time after a new user's decision is made.

So for each new user arriving to the system, we get a three dimensional matrix. The first dimension is the cell index, and the other two dimensions are the coordinates of the map.

\subsubsection{Utility Function of BE Users}

The utility function of the BE users is similar to that of the GBR users, except the load factor. The utility function of a BE user $k$ with a cell $j \in \mathcal{C}$ is formulated as

$$
U_{k j}(x, y)=p_{k}(x, y) \cdot s_{j}(x, y) \cdot \frac{\left(1-\rho_{j}^{b}(k)\right)}{n_{j}^{b}(k)+1}
$$


where $\rho_{j}^{b}(k)$, as defined in (5.14), is the load factor of cell $j$ of existing GBR users when the BE user $k$ arrives to the system. $n_{j}^{b}(k)$ is the number of the BE users in cell $j$ when user $k$ arrives to the system.

\subsubsection{Sequential Optimization}

Due to the fact that each user, or more precisely, each session, arrives to the system sequentially and it is inappropriate to suggest a user to move more than once during one data session, we use a greedy algorithm to optimize the utility function for each user sequentially based on the network situation at the moment the user arrives. Another benefit of the sequential optimization in this system is that the system knows the actions of the preceding users, or the system can predict their actions based on their probabilities of moving obtained from the completed optimization problems. Different from dynamic programming, the algorithm never reconsiders its choices, i.e., a user will not receive a second suggestion during one data session even though the network load has changed (and it may be better to move to another location) after he receives the first suggestion.

For a new GBR user $i$, we conduct a exhaustive search on the utility function $U_{i j}(x, y)$ of all the cells and locations based on the current load information. The optimization problem is formulated as

$$
\begin{array}{ll}
\max _{j, x, y} & U_{i j}(x, y) \\
\text { s.t. } & W_{j}-\sum_{i^{\prime} \in \mathcal{U}^{g}(i)} a_{i^{\prime} j} w_{i^{\prime} j}>\frac{r}{s_{j}(x, y)} \\
x & \in[0, X] \\
y & \in[0, Y] \\
& j \in \mathcal{C},
\end{array}
$$

where $x$ and $y$ take discrete values from the range $X$ and $Y$ based on the resolutions of the map. The matrix $a_{i^{\prime} j}$, which shows the association relation of all the existing GBR users to the associated cells at the moment the user $i$ arrives to the system, is a know matrix for each new user. The exhaustive search over the three dimensional matrix provides the optimal value for $U^{\star}$ for user $i$ and the corresponding values of the variables, i.e., the optimal associated cell $j^{\star}$ and the optimal location $\left(x^{\star}, y^{\star}\right)$. The probability of user $i$ to move to the optimal location $\left(x^{\star}, y^{\star}\right)$ is obtained from 
the $P$ function of this user, $p_{i}\left(x^{\star}, y^{\star}\right)$, which we denote as $p_{i}^{\star}$.

The first constraint in the problem guarantees that there are enough resources in the potential cell for user $i$ to reach rate $r$. If no cell is feasible because of the constrain, it means there is no location in any cells that can provide the spectral efficiency and the required resources to make this user reach the guaranteed bit rate, or such a location does exist yet this user has zero probability to move there based on our $P$ function (e.g., too far from the current location), an outage of this GBR user happens and no suggestion will be given.

As users only have probabilities to move within a short distance, i.e., p $(\mathrm{i}, \mathrm{j})$ equals to zero if the potential locations are far from the users, so it is realistic for the UIL controller to do the exhaustive search over all of the cells in a very short time and to provide suggestions to each user when the sessions start.

The optimization problem for the BE users is similar to the GBR users except that it comes without the first constraint that guarantees the amount of resources available as in (5.16).

In the simulation, the action of the user can be modeled as a Bernoulli trial. A random number between 0 and 1 is generated and is compared to the $p_{i}^{\star}$. This user moves if the random number is smaller than $p_{i}^{\star}$, stays otherwise. If user $i$ complies with the suggestion, the location of this user will be changed to $\left(x^{\star}, y^{\star}\right)$, and cell $j^{\star}$ will be assigned as the associated cell for this user, i.e., $a_{i j^{\star}}=1$. If the user chooses to stay without moving, he will be associated with the cell that provides the best SINR value. After the decision is made for each user, the association matrix $a_{i^{\prime} j}$ is updated as an input to calculate the load factor for the next user.

\subsubsection{An Example of Load Balancing Achieved by UIL}

In this section, we provide an example of a typical user associating with a cell based on the previously introduced utility function. A user is located as shown by the red cross in Figure 5.4 (a), and the probabilities of this user to move from the current location to other locations are shown in 5.4 (b). The probability $\mathrm{P}$ is calculated from Equation (5.9). A max-spectral-efficiency map is shown in Figure 5.4 (c), where each pixel is represented by the maximum value out of the spectral efficiencies received from all the cells, including macrocells and small-cells. Correlated shadowing for macrocells is used in the serving area and the log-normal shadowing is used in the wrap-around area. No shadowing is applied to small-cells. This map provides the 
natural selection of cell to associate with by the cell index from $\operatorname{argmax}\left(s_{p j}\right)$, where $s_{p j}$ is the spectral efficiency of pixel $p$ from cell $j$.

Based on the spectral efficiency map in Figure 5.4 (c), this user will associate with the macrocell A (as shown by the arrow in Figure 5.4 (a)) if best-SINR is the association strategy. If we multiply the $\mathrm{P}$ map with the max-spectral-efficiency map, i.e., without considering the load of cells, the user will be suggested to move to the small-cell B in the map.

By multiplying the spectral efficiency map of each cell, the load of each cell, and the probability of moving map of this user, we get a 3D utility map. To show the results in a $2 \mathrm{D}$ map, we represent each pixel with the maximum value of all the utilities among all the cells at each pixel, which is shown in Figure 5.4 (d).

From Figure 5.4 (d), we can see that cell B has very low utility and cell C and cell $\mathrm{D}$ have the highest utility. The high load is the reason why the utility of small-cell B becomes low. The user will be suggested to move to small-cell $\mathrm{C}$ or D according to the max-utility map in Figure $5.4(\mathrm{~d})$.

\subsubsection{Outlook: Include Mobility Model}

If a user mobility model is included in the system, Equation (5.14) should be modified to include network load variation during the time period that the current user is moving to the potential location. For example, when a new user $i$ arrives to the system at time $t$, the loads of the potential cells used in the utility function are not the loads at the moment $t, \rho_{j}(t), j \in C$, but the load at the moment when user $i$ arrives to the potential cell, $\rho_{j}\left(t+\tau_{j}\right), j \in C$, where $\tau_{j}$ is the time needed for user $i$ to move to cell $j$. The users considered in the load $\rho_{j}\left(t+\tau_{j}\right)$ should include the coming users and exclude the leaving users during the time interval $\tau_{j}$ for each cell $j$. With the probabilities of moving of the existing users available, the numerator in Equation (5.14) is then changed to the expected value of the resources consumed by the existing and the potential users.

\subsection{Load-Aware Cell Association}

In this section, we develop a baseline load balancing approach without involving users' movement. Users are associate with cells not purely based on the received 


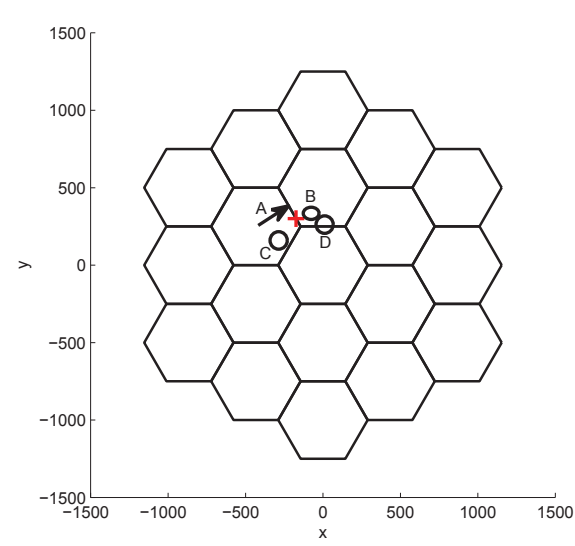

(a) User location

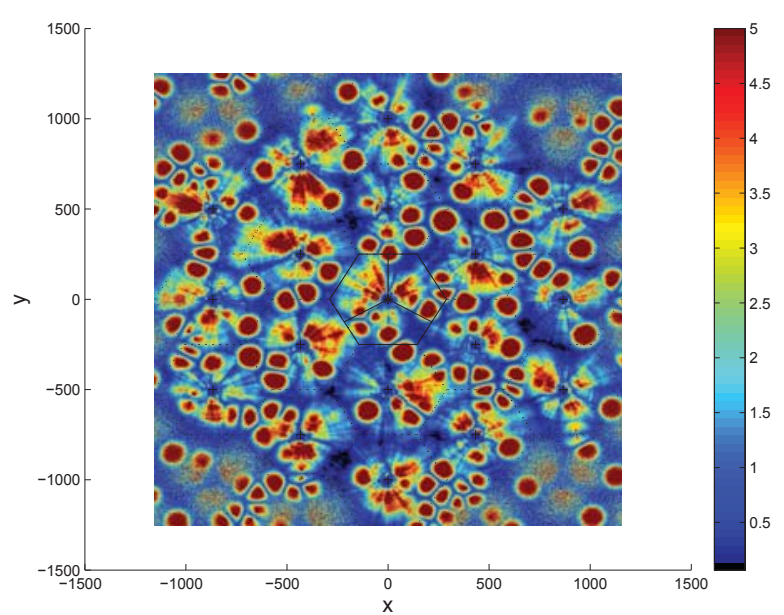

(c) Max-spetral-efficiency map

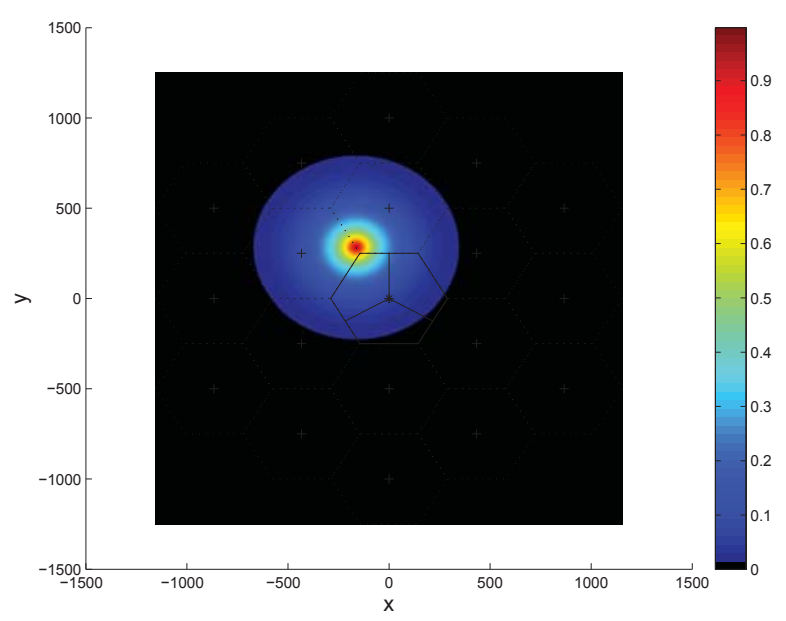

(b) Probability of moving

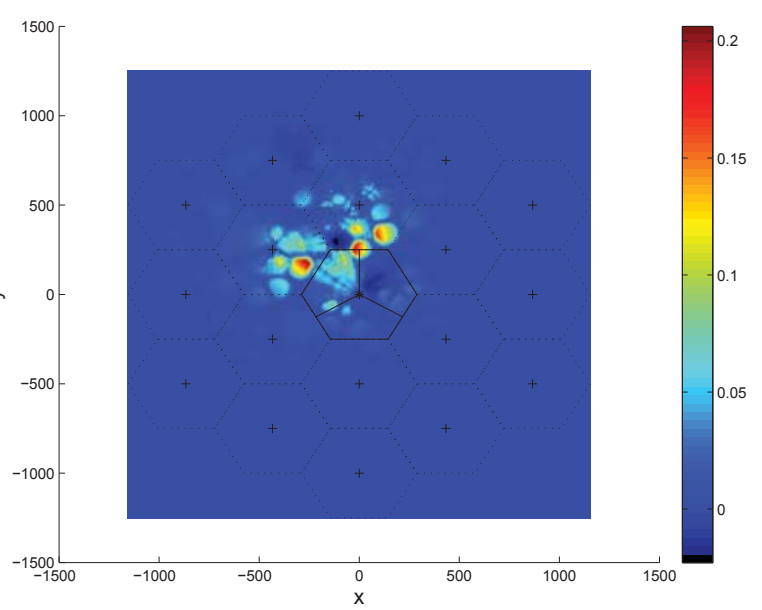

(d) Max-utility map

Figure 5.4: An example of load balancing achieved by UIL. The location of the user is shown in (a) as a red cross. The probability map of moving to other locations for this user in shown in (b). The user will associate will the macrocell A (shown as an arrow in (a)) if best-SINR is the association strategy according to the max-spectral-efficiency map in (c). The user will be suggested to move to the small-cell B if load is not considered. If we use the utility function defined in this thesis, the user will be suggested to move to the small-cell $\mathrm{C}$ or $\mathrm{D}$ according to the max-utility map in (d). The small-cell B has very low utility because it has a high load. 
SINR, but also on the load of each cell. To some extent, this is also an approach to push capacity supply to traffic demand through the process of cell association, known as load-aware cell association. Load-aware cell association has been researched in the literature intensively as an approach to perform load balancing, yet only one class of users is assumed (in most cases, best effort users). In this section, we formulate the load-aware association problem with the same user model and the same optimization method as in the UIL approach. The performance comparison with the UIL approach is provided in the next section.

\subsubsection{Related Work}

In [55], the handoff and cell association are formulated into an iterative optimization problem and solved with a distributed load-awareness scheme. In [43] and [45], with the utility function of the long-term user rate as the objective, the duality theory in optimization is used to tackle the cell association problem. The distributed solutions inside interpret the loads as the price of the BSs, which lead to a more balanced traffic load compared to the SINR-based cell association. In [56], a heuristic solution to the joint problem of cell association and linear beamformer design in a MIMO HetNets is given.

A typical load-aware cell association optimization problem [43] [45] is defined as

$$
\begin{array}{cl}
\max _{a_{i j}, n_{j}} & \sum_{i, j} a_{i j} \log \left(\frac{W_{j}}{n_{j}} \log \left(1+s_{i j}\right)\right) \\
\text { s.t. } & \sum_{j} a_{i j}=1 \\
& \sum_{i} a_{i j}=n_{j} \\
& \sum_{j} n_{j}=N \\
& a_{i j} \in 0,1
\end{array}
$$

where $n_{j}$ is the number of users associated with cell $j$, and $N$ is the total number of users in the system. In this formulation, the objective is the sum of each user's utility, $\log \left(r_{i j}\right)$, where $r_{i j}$ is the rate of user $i$ with cell $j$ calculated from the Shannon formula. The log-utility is a proportionally fair function, which encourages allocating more resources to the low-rate users. In other words, this property encourages load 
balancing. The authors in [43] claim that the optimal resource allocation strategy is equal allocation when log-utility is adopted, which is the reason why each user gets the amount of $\frac{W_{j}}{n_{j}}$ resources in Equation (5.17).

As mentioned before, only best effort users are modeled in this optimization problem, and resources are equally allocated among all users. To be comparable with the UIL approach we proposed in the previous section, we reformulated the loadaware association problem in the next section with the user model and the resource allocation scheme we defined in Section 5.3.

\subsubsection{Proposed Scheme}

Similar to the utility function defined for the UIL scheme, we define the utility functions for GBR users and BE users separately for load-aware cell association scheme. Without including the user moving, only two factors are considered in the function, the spectral efficiency and the load of the potential cell. The utility function of a GBR user $i$ with a cell $j$ is defined as

$$
\hat{U}_{i j}=s_{i j} \cdot\left(1-\rho_{j}^{b}(i)\right)
$$

where $s_{i j}$ denotes the spectral efficiency of cell $j$ at the current location of user $i$, and $\rho_{j}^{b}(i)$ has the same definition as in the UIL scheme, the load of GBR users of cell $j$ when user $i$ arrives to the system. Similarly, the utility function of a BE user $k$ with a cell $j$ is defined as

$$
\hat{U}_{k j}=s_{k j} \cdot \frac{\left(1-\rho_{j}^{b}(k)\right)}{n_{j}^{b}(k)+1} .
$$

For each new GBR user, an exhaustive search is conducted on all the cells to find the maximum utility defined in (5.18). The optimization problem is formulated as

$$
\begin{array}{ll}
\max _{j} & \hat{U}_{i j} \\
\text { s.t. } & W_{j}-\sum_{i^{\prime} \in \mathcal{U}^{g}(i)} a_{i^{\prime} j} w_{i^{\prime} j}>w_{i j} \\
& j \in \mathcal{C}
\end{array}
$$

The GBR user $i$ is associated to $j^{\star}$ from this optimization problem, or associated to the best-SINR cell if there is no cell feasible. The association matrix $a_{i j}$ is updated 
Table 5.1: Simulation parameters in Chapter 5

\begin{tabular}{|c|c|}
\hline Parameter & Assumption \\
\hline Macrocell layout & Hexagonal grid of $19 \times 3=57$ macrocells \\
\hline Picocell layout & $57 \times 2=114$ picocells $(\mathrm{BPP})$ \\
\hline Minimum distance between picocells & $40 \mathrm{~m}$ \\
\hline Average user number & 10 users $/$ cell $\times 171$ cells $=1710$ users \\
\hline Average session length & $300 \mathrm{~s}$ \\
\hline Guaranteed bit rate for GBR users & $1 \mathrm{Mbps}$ \\
\hline Percentage of GBR users & $50 \%$ \\
\hline System bandwidth & $10 \mathrm{MHz}(\mathrm{FDD})$ at $2 \mathrm{GHz}$ \\
\hline Shadowing & Log-normal, s.d. 4 for LOS, 6 for NLOS \\
\hline Macrocell Tx power & $46 \mathrm{dBm}$ \\
\hline Picocell Tx power & $37 \mathrm{dBm}$ \\
\hline Antenna gain & Macrocell: $17 \mathrm{dBi}$, picocell: $5 \mathrm{dBi}$ \\
\hline Traffic model & $\mathrm{BE}$ and GBR \\
\hline
\end{tabular}

after each user's associated cell is obtained.

For a BE user $k$, the associated cell is $\operatorname{argmax}_{j} \hat{U}_{k j}$ with no constrains.

\subsection{Numerical Results}

\subsubsection{Simulation Setup}

In this simulation, ITU urban macrocell (UMa) [57] and 3GPP case 6.2 in release 9 [18] is the scenario used for HetNets. 19 macrocells sites, each of 3 cells with inter site distance (ISD) of 500 meters, are configured in the system. The locations of the macrocell sites are fixed and form a hexagonal grid layout. The HetNets consists of two tiers with outdoor picocells overlaid on the same area of macrocells. The distribution of picocells is random and uniform. A wrap-around technique is applied on both macro and small-cells to eliminate the boundary effect.

The channel follows the model 2 in [18] for both macrocells and outdoor picocells, in which a LOS and NLOS power-law path loss model is used. The downlink 
signal experiences path loss and shadowing, while the fast fading is assumed to be averaged out. The shadowing is correlated based on the models used in [58] [59]. An illustration of the correlated shadowing can be found in Figure 5.4 (c). The user rate is calculated from the product of resource allocated and the spectral efficiency reached, both of which are defined in Section 5.3.2. A user suffers interference from all the macrocells and picocells outside its own serving cell (which may be a macrocell or a picocell). Both macrocells and picocells share the same bandwidth, and no interference coordination or cancellation technique is used. Table 5.1 shows the key parameters used in the simulations.

Users arrive the system according to a Poisson process and leave the system after a session length. In each drop of the simulation, the system starts from zero users. Section 5.6.2 defines the transient and steady state of the simulation, and the performance evaluation inside is time-dependent. After that, all the performances are evaluated based on the snapshots of the system when the user number is in steady state, and each snapshot is taken from an individual drop, i.e., the snapshots are totally independent.

The metrics are evaluated with respect to the increasing spatial traffic demand heterogeneities under three scenarios: (a) no load balancing with best-SINR association strategy, (b) load balancing approach with load-aware association strategy as introduced in Section 5.5, and (c) load balancing approach with the UIL scheme. We change the value of $\sigma$ in LGCP to get user distributions with different spatial heterogeneities, which are measured by $C$, the normalized $\mathrm{CoV}$ of Voronoi cell area in the Voronoi tessellations of the user points [24]. When $C$ is equal to 1 , the user distribution forms a PPP.

In the UIL scheme, the probability of moving $p$ is the function of user traffic class (GBR or BE) and the moving distance. We adopt the result in [48], in which exponential functions for different traffic classes with respect to moving distances are obtained based on a survey result. The functions are shown in Section 5.3.3. In this thesis, we use the discount as the incentive method, and the incentive index is fixed at 4 , which is $80 \%$ discount if a user complies with the suggestion. Further work regarding different levels of incentive can be included. For a user, GBR or BE, the probability of moving $p^{\star}$ is derived from the distance between the current location and the optimal location $\left(x^{\star}, y^{\star}\right)$. A Bernoulli trial is then conducted to determine whether this user complies with the suggestion or not. If complies, the user will be 


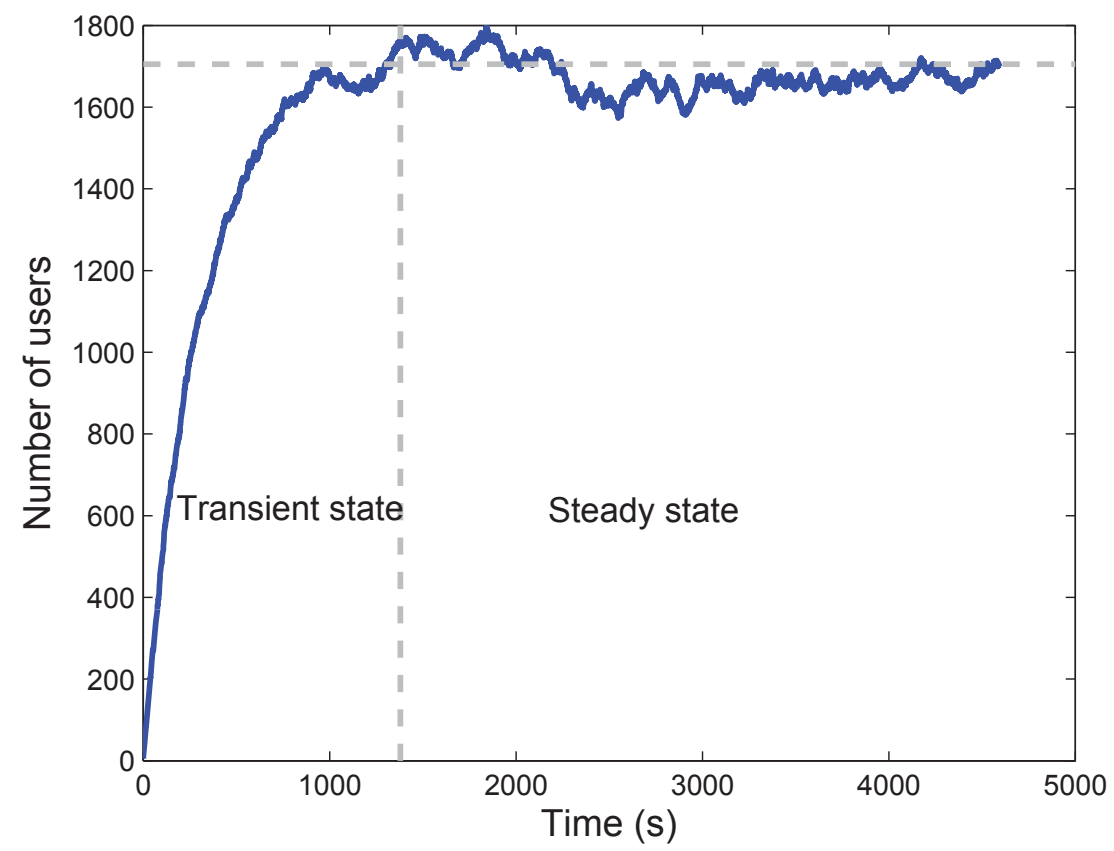

Figure 5.5: The number of users in the system over time in one drop.

relocated to $\left(x^{\star}, y^{\star}\right)$ and associated with cell $j^{\star}$ as discussed in Section 5.4.3. If not, the user will associate with a cell based on the best-SINR policy.

\subsubsection{Transient and Steady State Behavior}

The system starts from zero users, and users arrive to the system with exponential inter-arrival time. We fix the session length at 300 seconds, and users leave the system after the session expires. In the steady state, the average user number is 1710, i.e., 10 users per cell. Based on the queue theory, the arrival rate and the departure rate are calculated from the average number of users divided by the session length.

The system starts from transient state till the number of users reaches in a certain probability of error regarding to the average user number. Figure 5.5 shows the number of users changing over time and the transient time accordingly. An exponential fitting with coefficient $\lambda$ can be found from the nonlinear regression of the curve in 5.5. The transient time $\tau$ is calculated from the equation $1-e^{-\lambda \tau}=1-\epsilon$, where $\epsilon$ is the probability of error. In this simulation, we take $\epsilon$ as $1 \%$. Other statistics 


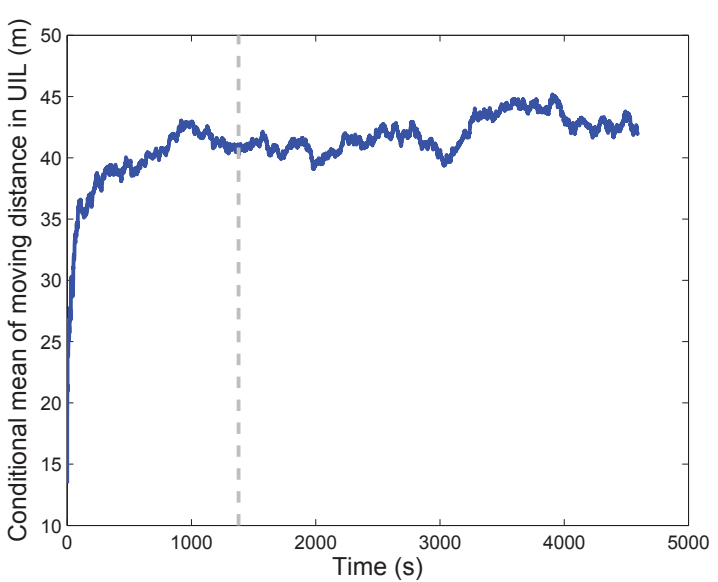

(a) Average moving distance

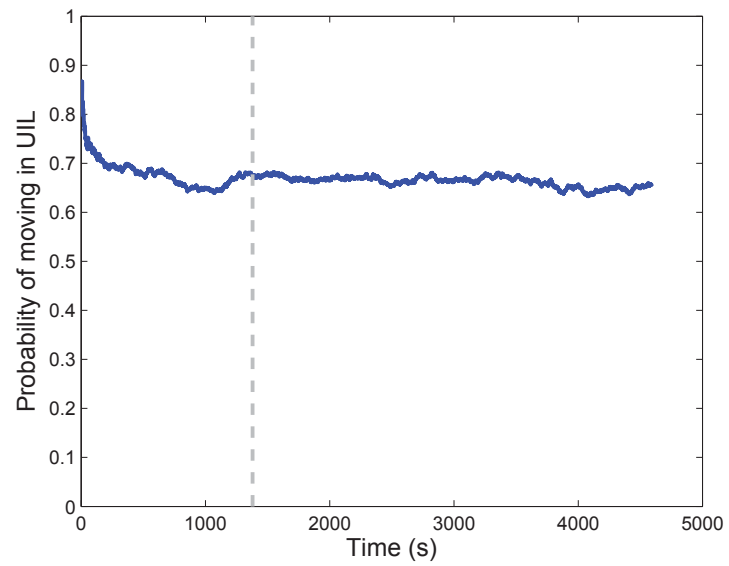

(b) Average moving probility

Figure 5.6: Statistics of user movement over time.

including average moving distance (conditional, only the users that move are considered) and the moving probability are shown in Figure 5.6. As we can see from Figure 5.6, the average moving distance is about 43 meters, and the probability of moving is about 0.7, which complies with the curves in Figure 5.2 (a) with $80 \%$ discount as the incentive.

The user spatial heterogeneity $(\mathrm{CoV})$ is evaluated with and without UIL. As we can see from Figure 5.7, the CoV of user spatial distribution is lower in the UIL scheme. This is due to the fact that UIL suggest users to move to the less loaded cell, which helps to reduce the degree of user spatial heterogeneity.

The performances hereafter are evaluated based on the snapshots of the system when the user number is in steady state, and each snapshot is taken from an independent drop.

\subsubsection{Loads among Different Cells}

The load factor of GBR users is defined as $\rho$ in Equation (5.14) in Section 5.4.1. If we take the standard deviation of $\rho_{j}(j \in C)$, denoted as $s t d_{\rho}$, it can be used as a measure to indicate the degree of network-wide load balance. The lower the $s t d_{\rho}$ is, the more balanced the network is. Figure 5.8 shows the $s t d_{\rho}$ with respect to user spatial heterogeneities under different load balancing strategies. First, it is obvious that the 


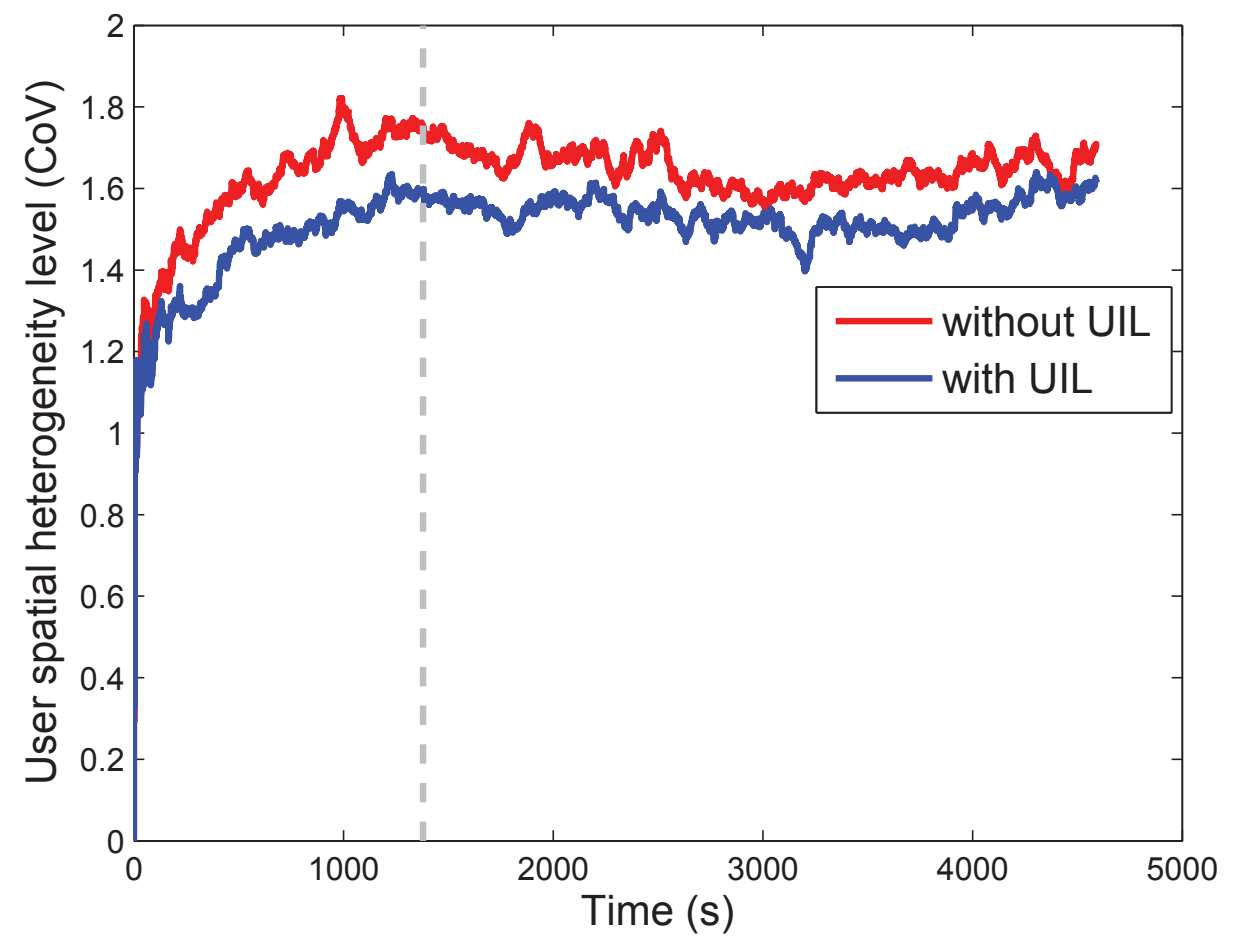

Figure 5.7: The CoV of user spatial distribution over time with and without UIL. 


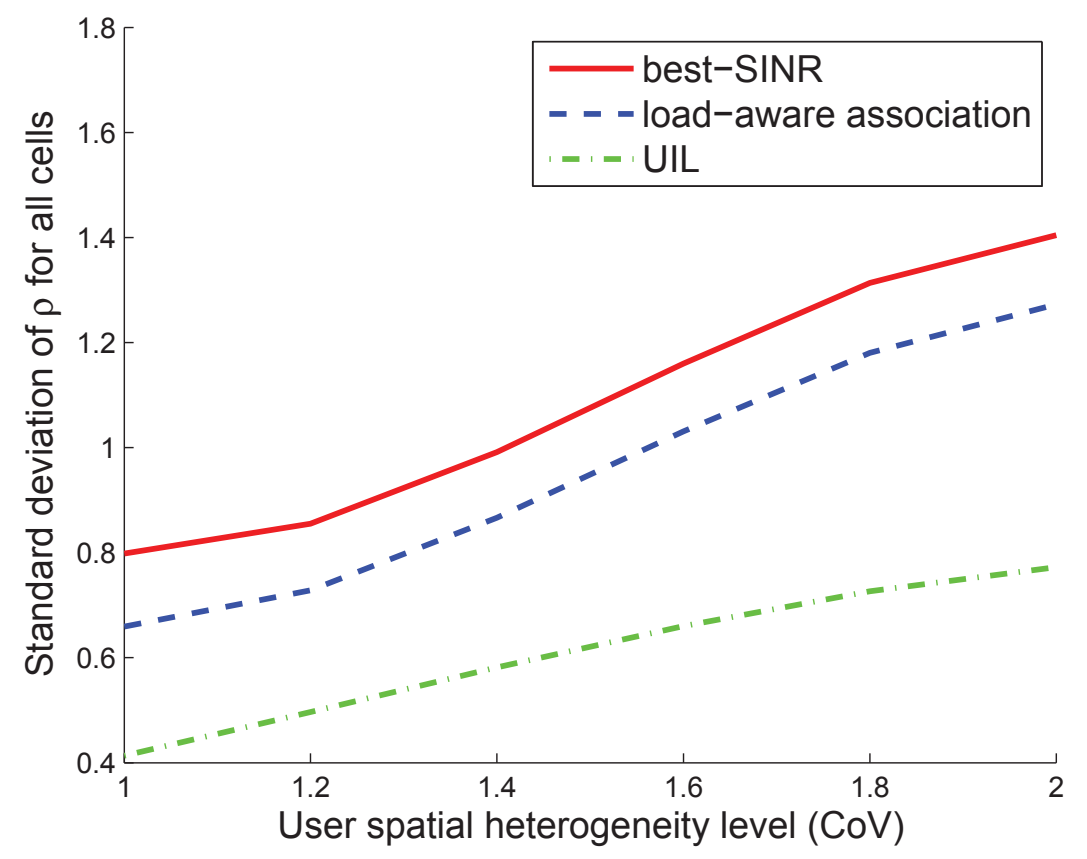

Figure 5.8: The standard deviation of GBR user load $(\rho)$ of all the cells with respect to user spatial heterogeneities under different load balancing strategies.

network is becoming more imbalanced when the user spatial heterogeneity (traffic demand heterogeneity) increases in all the three schemes. Second, as we can see, $s t d_{\rho}$ is the lowest in the UIL scheme, highest in the best-SINR scheme, and the load-aware cell association scheme is in the middle, which shows that the UIL approach improves network-wide load balance substantially, and the load-aware association approach is also an effective approach to do load-balancing compare to the best-SINR strategy without load balancing.

\subsubsection{GBR Users Outage}

As shown in Figure 5.9, the outage percentage is the lowest (best) with the UIL scheme and the highest (worst) with the best-SINR strategy. The load-aware association strategy as an approach to load balancing without the involvement of user relocation performs in the middle. The outage percentages rise quickly with the increase of user spatial heterogeneity (i.e., traffic demand heterogeneity) in all the three strategies. 


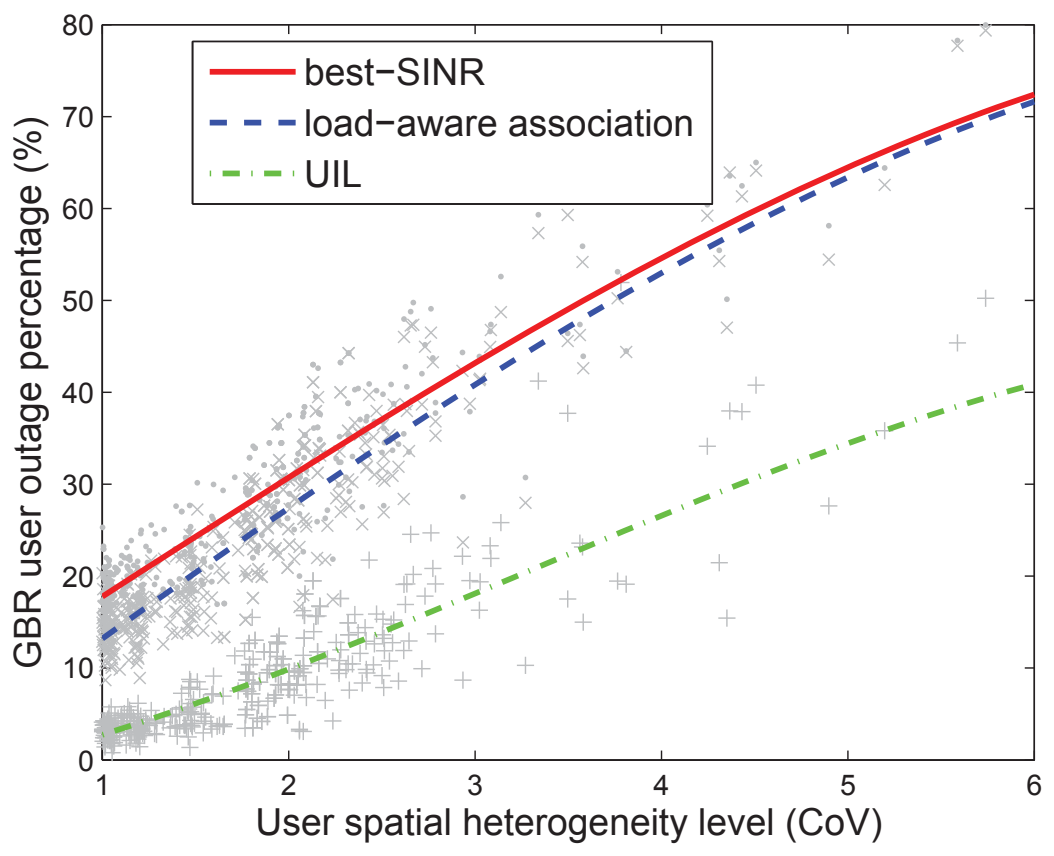

Figure 5.9: The outage percentage of GBR users with respect to user spatial heterogeneities under different load balancing strategies. Each dot, cross, and plus sign represents the result of a simulation drop in best-SINR, load-aware cell association and UIL scheme, respectively. The lines are the polynomial curve fitting with $n=3$. 


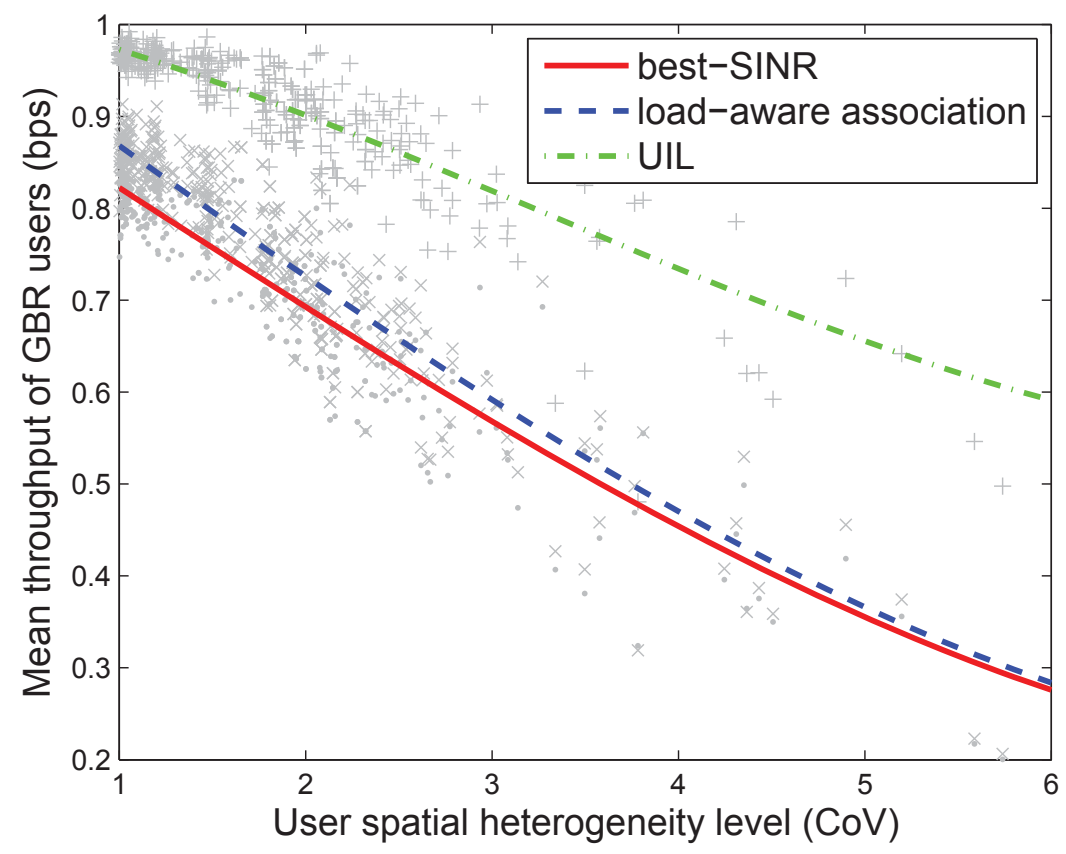

Figure 5.10: Mean rate of all the GBR users in the system with respect to user spatial heterogeneities under different load balancing strategies. Each dot, cross, and plus sign represents the result of a simulation drop in best-SINR, load-aware cell association and UIL scheme, respectively. The lines are the polynomial curve fitting with $n=3$.

The reason is when the user spatial heterogeneity increases, the traffic demand may be highly clustered in some areas that exceed the capacities of the associated cells, which results in higher GBR user outage. Load-aware cell association strategy takes the load factor into consideration, and thus performs better than the best-SINR strategy without load balancing. The UIL scheme outperforms the load-aware association by combining spatial relocation (with probability) and load factor together.

\subsubsection{Mean User Rate}

The mean user rates of GBR users and BE users are calculated separately as shown in Figure 5.10 and Figure 5.11, respectively. They all have the same downward trends with the increase of user spatial heterogeneity. For GBR users, the UIL scheme performs the best, the load-aware association strategy comes next, and the bestSINR strategy with no load balancing performs the worst. This can be explained 


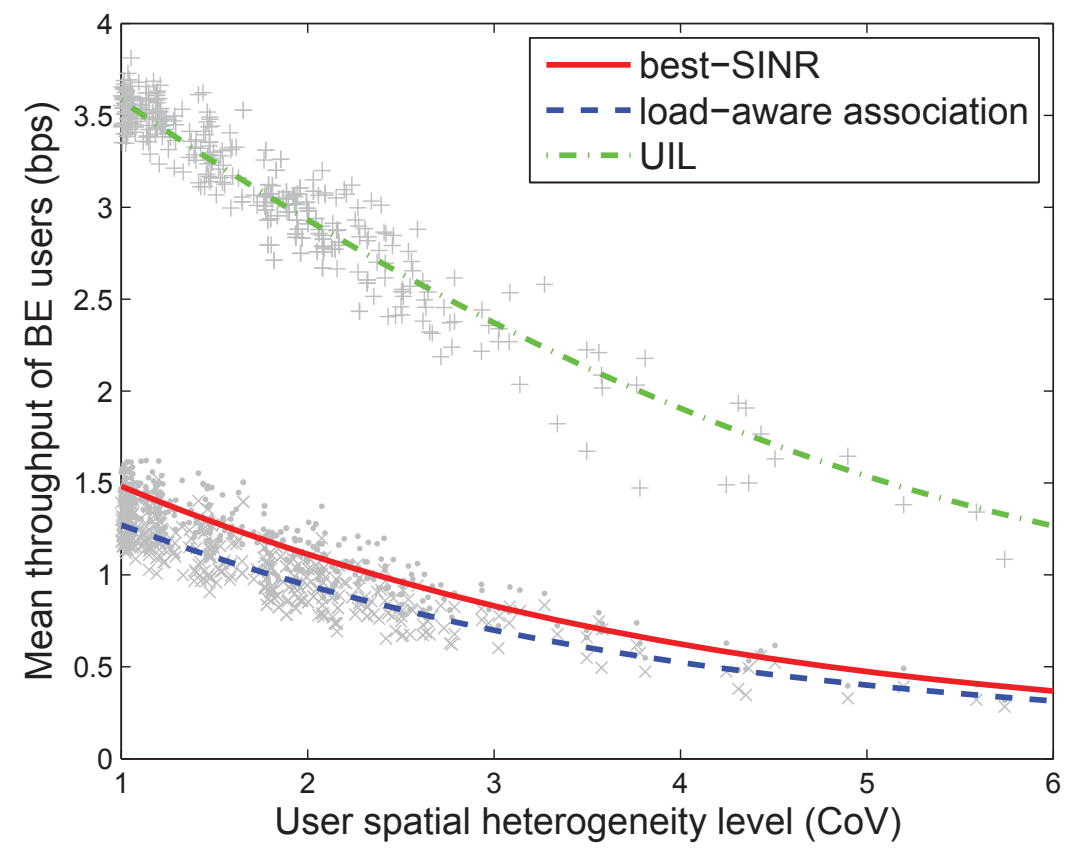

Figure 5.11: Mean rate of all the BE users in the system with respect to user spatial heterogeneities under different load balancing strategies. Each dot, cross, and plus sign represents the result of a simulation drop in best-SINR, load-aware cell association and UIL scheme, respectively. The lines are the polynomial curve fitting with $n=3$.

with the same reason mentioned for the outage percentage. However, for BE users, the UIL scheme still performs the best but the load-aware cell association strategy does not surpass the best-SINR strategy. This is due to the fact that the loadaware strategy may associate a GBR user to a lightly loaded neighbor cell with lower spectral efficiency received, which results in a higher resource consumption. Because of the priority of GBR users, less resources are left for BE users, which brings a worse performance for the load-aware association strategy in terms of mean user rate.

\subsubsection{Fairness Index}

As in Section 4.3.2, we use Jain's index to measure the fairness of user rate. Different from Section 4.3.2, GBR users and BE users are evaluated separately. Figure 5.12 and Figure 5.13 show the fairness index of GBR user rate and BE user rate versus user spatial heterogeneity under different network configurations, respectively. Similar 


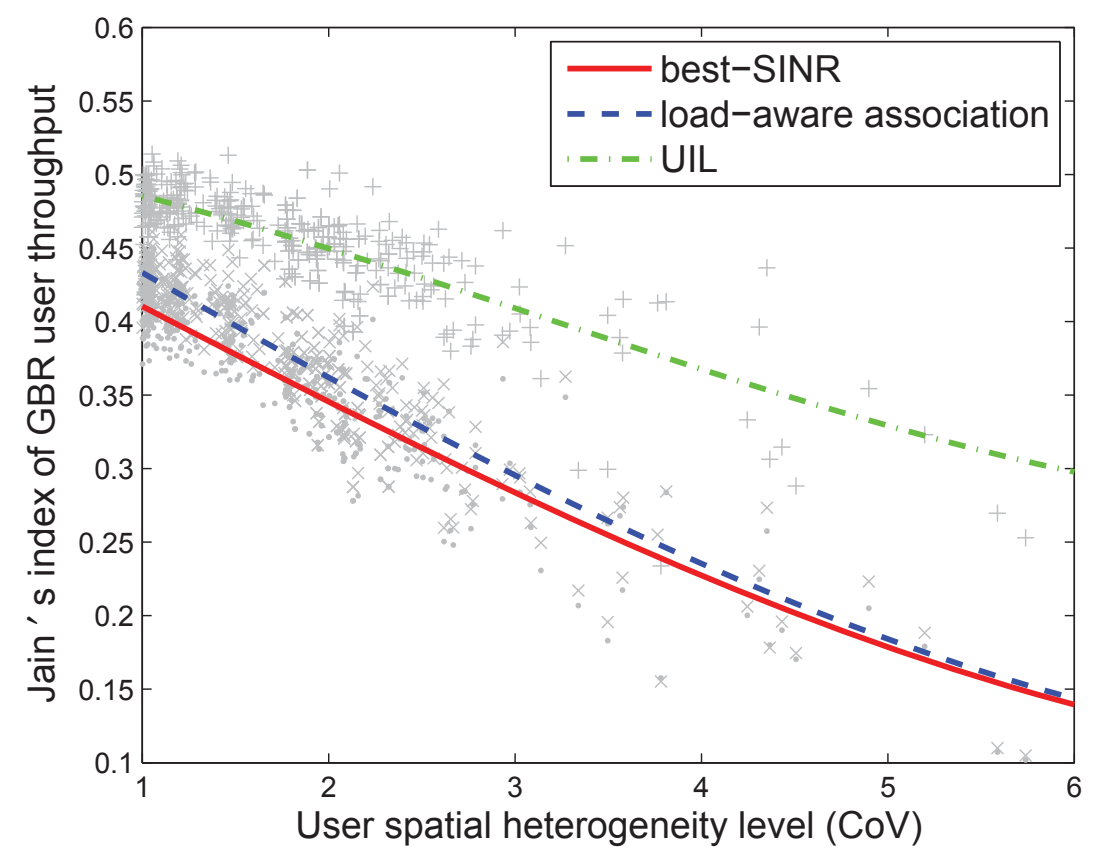

Figure 5.12: Jain's index of rate of all the GBR users in the system with respect to user spatial heterogeneities under different load balancing strategies.

to the user rate, the UIL shceme performs the best, while load-aware association performs better than best-SINR with GBR users, and worse with BE users. This is because the load-aware cell association strategy introduced in this thesis provides higher priority for GBR users.

\subsection{Summary}

In this section, a novel load balancing approach in cellular networks is proposed as the second approach to load balancing. User-in-loop as the spatial traffic shaping method is the enabler of the approach, in which the traffic demand (users) is pushed to the capacity supply (BSs).

A user application model consisting of GBR and BE is considered in this chapter with corresponding resource allocation policy. To better evaluate the performance, a load-aware cell association strategy is introduced with the same user traffic model and resource allocation policy as the UIL scheme. Numerical results show that the 


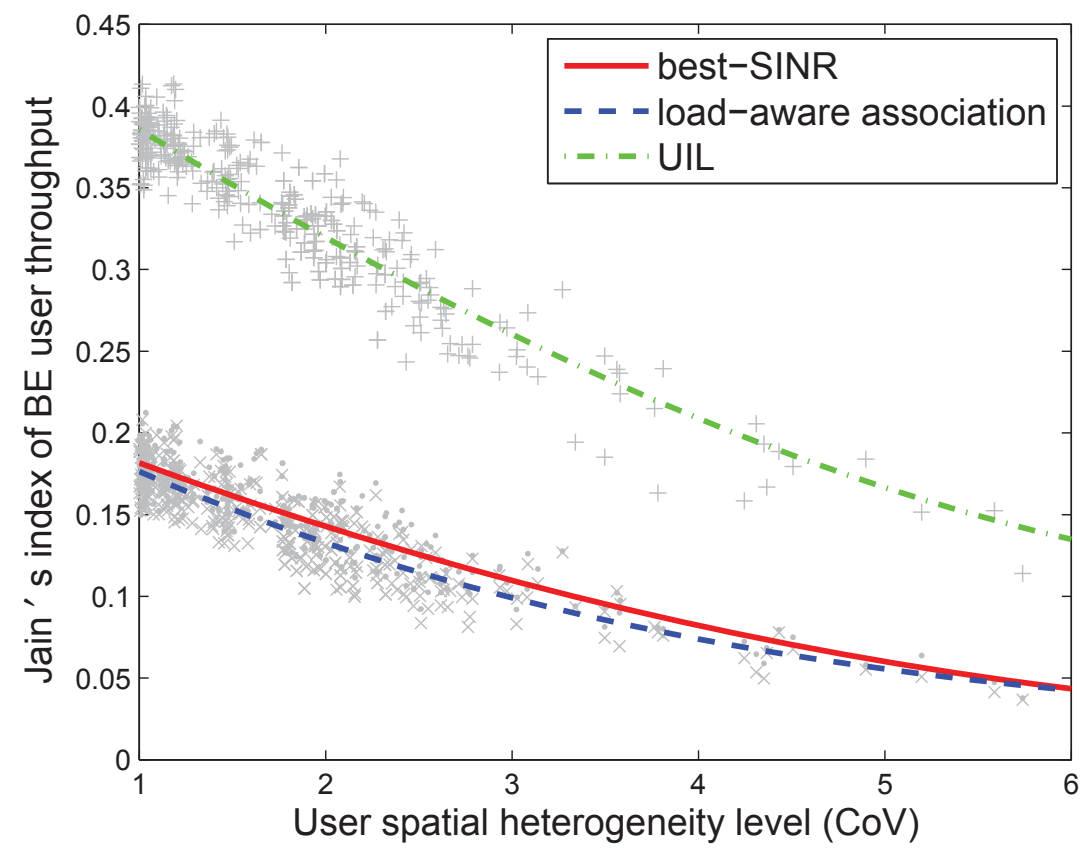

Figure 5.13: Jain's index of rate of all the BE users in the system with respect to user spatial heterogeneities under different load balancing strategies.

proposed load balancing approach with UIL outperforms the load balancing approach with load-aware cell association strategy and the non-load-balancing approach with best-SINR association strategy significantly. 


\section{Chapter 6}

\section{Conclusion and Future Work}

\subsection{Conclusion}

Cellular networks are becoming increasingly heterogeneous in two different dimensions. First in the capacity supply dimension, the architecture of the network is becoming increasingly heterogeneous, with small-cells (such as picocells and femtocells) layered upon traditional macrocells. Second, from the traffic demand side, new applications with diversified traffic patterns are emerging everyday with the proliferation of smart mobile devices (e.g., smart phones and tablets). In this thesis, we use the term "HetHetNets" to denote the cellular networks that have heterogeneous capacity supply and heterogeneous traffic demand.

Instead of being one dimensional heterogeneous in the network side (capacity supply) as in the literature, the research in this thesis is taken under HetHetNets, with emphasis on user side (traffic demand).

This thesis raises three questions: (1) How to model the heterogeneous user distribution in spatial domain (heterogeneous traffic demand)? (2) What is the impact of user spatial heterogeneity on heterogeneous cellular networks (impact of hetero-

geneous traffic demand on heterogeneous capacity supply)? (3) What are the solutions to combat the load imbalance between the traffic demand and the capacity supply in HetHetNets?

To answer the first question, users are modeled with a doubly stochastic Poisson process, which models the user spatial distribution from homogeneous to extremely heterogeneous with a single parameter. The degree of the spatial heterogeneity is measured by a single positive real number $C$ based on the Voronoi and Delaunay tessellations, an analogy of the inter-arrival time measure in one dimensional time 
domain. Besides being spatially heterogeneous, the user traffic is also heterogeneous in this thesis. Finite rate demand model is investigated compared to the commonly used best effort user model. Two user traffic classes, which are guaranteed bit rate and best effort, are further investigated with the corresponding resource allocation scheme that provides priority to the GBR users.

The second question is partly answered in the next section. The impact of user spatial heterogeneity (captured by $C$ ) on the performance of downlink cellular networks is obtained. We find that the network performance metrics deteriorate significantly with increasing $C$ if the user locations are uncorrelated with the locations of the macro and small-cell BSs.

Cluster analysis on the non-uniform user points is utilized to find the cluster centroids as the potential locations for small-cells. A selection method that considers the relative distance between user to macrocells and user to potential small-cells is introduced to choose the clusters to deploy small-cells. Simulation results show that the network performance can improve substantially with increasing $C$ if we take advantage of user spatial heterogeneity to deploy small-cells in the appropriate locations. This is the first approach to the third question by pushing the capacity supply to the traffic demand, and it also answers the second question when the locations of BSs and users are correlated.

Other than pushing capacity supply to traffic demand, a novel traffic-shaping load balancing approach in cellular networks through the recently developed user-in-theloop (UIL) concept is proposed. In this approach, users are encouraged to move to a better location that has higher spectral efficiency and/or a lower traffic load. The utility function proposed in this approach jointly combines the spectral efficiency, the traffic load and the probability of user moving. To better evaluate the performance, a load-aware cell association strategy is introduced with the same user traffic model and resource allocation policy as in the UIL scheme. Numerical results show that the proposed load balancing approach with UIL outperforms the load balancing approach with load-aware cell association strategy and the non-load-balancing approach with best-SINR association strategy significantly. This is the second approach to deal with the third question by pushing the traffic demand to the capacity supply. 


\subsection{Future Work}

\subsubsection{Pushing Capacity Supply to Traffic Demand}

The cluster analysis approach to find small-cell locations in this thesis can also be used to locate the user relays that are to be activated to serve the users around in $5 \mathrm{G}$ networks. Weighted $k$-means clustering can be applied to model the users with different rate demand. The correlation among users and the cross-correlation between the users and BSs can also be included in the user spatial model [60]. As LGCPs are tractable for mathematical analysis [61], future work may involve analytical expressions for network performance.

\subsubsection{Pushing Traffic Demand to Capacity Supply}

The proposed load balancing approach can be extended with the temporal UIL. Similar to the policies that have been implemented in power supply companies, temporal UIL encourages users to postpone a heavy data application in busy hours. Limited back-haul can also be included in the model. More incentive methods should also be investigated in the UIL approach for load balancing. With the machine type of communications becoming more popular, the UIL concept can be extended to the machine nodes, which are easier to be controlled to move compared to mankind users. A different utility function taking other possible factors or with different weights of the factors can also be explored. 


\section{List of References}

[1] A. Damnjanovic, J. Montojo, Y. Wei, T. Ji, T. Luo, M. Vajapeyam, T. Yoo, O. Song, and D. Malladi, "A survey on 3GPP heterogeneous networks," Wireless Communications, IEEE, vol. 18, pp. 10-21, June 2011.

[2] S. Landström, A. Furuskãr, K. Johansson, L. Falconetti, and F. Kronestedt, "Heterogeneous networks-increasing cellular capacity," Ericsson Review, p. 4, 2011.

[3] M. Mirahsan, R. Schoenen, and H. Yanikomeroglu, "HetHetNets: Heterogeneous traffic distribution in heterogeneous wireless cellular networks," IEEE Journal on Selected Areas in Communications, Special Issue on Recent Advances in Heterogeneous Cellular Networks, July 21, 2014. under review.

[4] J. Andrews, "Seven ways that HetNets are a cellular paradigm shift," IEEE Communications Magazine, vol. 51, no. 3, pp. 136-144, 2013.

[5] H. ElSawy, E. Hossain, and M. Haenggi, "Stochastic geometry for modeling, analysis, and design of multi-tier and cognitive cellular wireless networks: A survey," IEEE Communications Surveys \& Tutorials, vol. 15, no. 3, pp. 9961019, 2013.

[6] H. Dhillon, R. Ganti, F. Baccelli, and J. Andrews, "Modeling and analysis of ktier downlink heterogeneous cellular networks," IEEE Journal on Selected Areas in Communications, vol. 30, no. 3, pp. 550-560, 2012.

[7] M. Haenggi, Stochastic geometry for wireless networks. Cambridge University Press, 2012.

[8] D. Stoyan, W. Kendall, and J. Mecke, Stochastic Geometry and Its Applications. John Wiley \& Sons, 2nd edition ed., 1995.

[9] M. Elalem and L. Zhao, "Realistic user distribution and its impact on capacity and coverage for a WCDMA mobile network," in Proc. IEEE Sarnoff Symposium, Mar. 2009.

[10] M. Taranetz and M. Rupp, "Performance of femtocell access point deployments in user hot-spot scenarios," in Proc. Australasian Telecommunication Networks and Applications Conference, Nov. 2012. 
[11] H. Dhillon, R. Ganti, and J. Andrews, "Modeling non-uniform UE distributions in downlink cellular networks," IEEE Wireless Communications Letters, vol. 2, no. 3, pp. 339-342, 2013.

[12] R. Ganesh and K. Joseph, "Effect of non-uniform traffic distributions on performance of a cellular CDMA system," in Proc. IEEE International Conference on Universal Personal Communications (ICUPC), vol. 2, pp. 598-602, Oct. 1997.

[13] B. Blaszczyszyn and D. Yogeshwaran, "Clustering comparison of point processes with applications to random geometric models," arXiv preprint arXiv:1212.5285, 2012.

[14] V. Lucarini, "From symmetry breaking to poisson point process in 2d voronoi tessellations: the generic nature of hexagons," Journal of Statistical Physics, vol. 130, no. 6, pp. 1047-1062, 2008.

[15] J. Rataj, I. Saxl, and K. Pelikán, "Convergence of randomly oscillating point patterns to the poisson point process," Applications of Mathematics, vol. 38, no. 3, pp. 221-235, 1993.

[16] L. Kleinrock and J. Silvester, "Optimum transmission radii for packet radio networks or why six is a magic number," in Conference Record, National Telecommunications Conference, (Birmingham, Alabama), pp. 4.3.2-4.3.5, December 1978.

[17] J. G. Andrews, F. Baccelli, and R. K. Ganti, "A tractable approach to coverage and rate in cellular networks," Communications, IEEE Transactions on, vol. 59, no. 11, pp. 3122-3134, 2011.

[18] 3GPP, "E-UTRA: Further advancements for E-UTRA physical layer aspects (release 9)," TR 36.814, 3GPP, Mar. 2010.

[19] H. ElSawy and E. Hossain, "Two-tier HetNets with cognitive femtocells: Downlink performance modeling and analysis in a multichannel environment," IEEE Transactions on Mobile Computing, vol. 13, pp. 649-663, Mar. 2014.

[20] V. Paxson and S. Floyd, "Wide area traffic: the failure of poisson modeling," Networking, IEEE/ACM Transactions on, vol. 3, pp. 226-244, Jun 1995.

[21] D. Alberto, P. Antonio, S. R. Pierluigi, P. Francesco, and V. Giorgio, "Internet traffic modeling by means of hidden markov models," Computer Networks, vol. 52, no. 14 , pp. $2645-2662,2008$.

[22] Y. Xie, J. Hu, Y. Xiang, S. Yu, S. Tang, and Y. Wang, "Modeling oscillation behavior of network traffic by nested hidden markov model with variable stateduration," Parallel and Distributed Systems, IEEE Transactions on, vol. 24, pp. 1807-1817, Sept 2013.

[23] J. Møller, A. R. Syversveen, and R. P. Waagepetersen, "Log Gaussian cox processes," Scandinavian Journal of Statistics, vol. 25, no. 3, pp. 451-482, 1998. 
[24] M. Mirahsan, Z. Wang, R. Schoenen, H. Yanikomeroglu, and M. St-Hilaire, "Unified and non-parameterized statistical modeling of temporal and spatial traffic heterogeneity in wireless cellular networks," in Proc. IEEE International Conference on Communications (ICC) Workshop on 5G Technologies, (Sydney, Australia), June 2014.

[25] P. J. Clark and F. C. Evans, "Distance to nearest neighbor as a measure of spatial relationships in populations," Ecology, pp. 445-453, 1954.

[26] C. D. Barr, Applications of Voronoi tessellations in point pattern analysis. ProQuest, 2008.

[27] J. Møller and R. P. Waagepetersen, "Modern statistics for spatial point processes*," Scandinavian Journal of Statistics, vol. 34, no. 4, pp. 643-684, 2007.

[28] E. Gilbert, "Random subdivisions of space into crystals," The Annals of mathematical statistics, pp. 958-972, 1962.

[29] K. Borovkov and D. Odell, "Simulation studies of some Voronoi point processes," Acta Applicandae Mathematicae, vol. 96, no. 1-3, pp. 87-97, 2007.

[30] M. Tanemura, "Statistical distributions of poisson voronoi cells in two and three dimensions," FORMA-TOKYO-, vol. 18, no. 4, pp. 221-247, 2003.

[31] L. Muche, "Distributional properties of the three-dimensional poisson delaunay cell," Journal of statistical physics, vol. 84, no. 1-2, pp. 147-167, 1996.

[32] P. Rathie, "On the volume distribution of the typical poisson-delaunay cell," Journal of applied probability, pp. 740-744, 1992.

[33] R. Miles, "The random division of space," Advances in applied probability, pp. 243-266, 1972.

[34] M. ITU-R, "2135," Guidelines for evaluation of radio interface technologies for IMT advanced, 2008.

[35] R. Schoenen and A. Otyakmaz, "Qos and flow management for future multi-hop mobile radio networks," in Vehicular Technology Conference Fall (VTC 2010Fall), 2010 IEEE 72nd, pp. 1-5, Sept 2010.

[36] H.-B. Chang and I. Rubin, "Impact of placement of small cells on downlink performance for cellular wireless networks," in Vehicular Technology Conference (VTC Fall), 2013 IEEE 78th, pp. 1-5, Sept 2013.

[37] H.-C. Lu, W. Liao, and F.-S. Lin, "Relay station placement strategy in ieee 802.16j wimax networks," Communications, IEEE Transactions on, vol. 59, pp. 151-158, January 2011.

[38] W. Guo and T. O'Farrell, "Relay deployment in cellular networks: Planning and optimization," Selected Areas in Communications, IEEE Journal on, vol. 31, pp. 1597-1606, August 2013. 
[39] W. Guo and S. Wang, "Interference-aware self-deploying femto-cell," Wireless Communications Letters, IEEE, vol. 1, pp. 609-612, December 2012.

[40] X. Li, X. Tang, C.-C. Wang, and X. Lin, "Gibbs-sampling-based optimization for the deployment of small cells in $3 \mathrm{~g}$ heterogeneous networks," in Modeling Optimization in Mobile, Ad Hoc Wireless Networks (WiOpt), 2013 11th International Symposium on, pp. 444-451, May 2013.

[41] P. Tan, M. Steinbach, and V. Kumar, Introduction to Data Mining. AddisonWesley Longman Publishing Co., Inc., 1st ed., 2005.

[42] J. Andrews, S. Singh, Q. Ye, X. Lin, and H. Dhillon, "An overview of load balancing in hetnets: old myths and open problems," Wireless Communications, IEEE, vol. 21, pp. 18-25, April 2014.

[43] Q. Ye, B. Rong, Y. Chen, M. Al-Shalash, C. Caramanis, and J. Andrews, "User association for load balancing in heterogeneous cellular networks," Wireless Communications, IEEE Transactions on, vol. 12, pp. 2706-2716, June 2013.

[44] K. Son, S. Chong, and G. Veciana, "Dynamic association for load balancing and interference avoidance in multi-cell networks," Wireless Communications, IEEE Transactions on, vol. 8, pp. 3566-3576, July 2009.

[45] K. Shen and W. Yu, "Downlink cell association optimization for heterogeneous networks via dual coordinate descent," in Acoustics, Speech and Signal Processing (ICASSP), 2013 IEEE International Conference on, pp. 4779-4783, May 2013.

[46] R. Schoenen and H. Yanikomeroglu, "User-in-the-loop: Spatial and temporal demand shaping for sustainable wireless networks," IEEE Communications Magazine, vol. 52, pp. 196-203, Feb. 2014.

[47] R. Schoenen, H. Yanikomeroglu, and B. Walke, "User in the loop: Mobility aware users substantially boost spectral efficiency of cellular ofdma systems," Communications Letters, IEEE, vol. 15, pp. 488-490, May 2011.

[48] R. Schoenen, G. Bulu, A. Mirtaheri, T. Beitelmal, and H. Yanikomeroglu, "Quantified user behavior in user-in-the-loop spatially and demand controlled cellular systems," in European Wireless, 2012. EW. 18th European Wireless Conference, pp. 1-8, April 2012.

[49] R. Schoenen and H. Yanikomeroglu, "Erlang analysis of cellular networks using stochastic petri nets and user-in-the-loop extension for demand control," in Globecom Workshops (GC Wkshps), 2013 IEEE, pp. 298-303, Dec 2013.

[50] R. Schoenen and H. Yanikomeroglu, "Dynamic demand control with differentiated qos in user-in-the-loop controlled cellular networks," in Vehicular Technology Conference (VTC Spring), 2013 IEEE 7rth, pp. 1-6, June 2013.

[51] R. Schoenen and H. Yanikomeroglu, "Economics of user-in-the-loop demand control with differentiated qos in cellular networks," in Personal Indoor and Mobile Radio Communications (PIMRC), 2012 IEEE 23rd International Symposium on, pp. 1131-1136, Sept 2012. 
[52] R. Schoenen, G. Bulu, A. Mirtaheri, T. Beitelmal, and H. Yanikomeroglu, "First survey results of quantified user behavior in user-in-the-loop scenarios for sustainable wireless networks," in Vehicular Technology Conference (VTC Fall), 2012 IEEE, pp. 1-5, Sept 2012.

[53] R. Schoenen, G. Bulu, A. Mirtaheri, and H. Yanikomeroglu, "Green communications by demand shaping and user-in-the-loop tariff-based control," in Online Conference on Green Communications (GreenCom), 2011 IEEE, pp. 64-69, Sept 2011.

[54] R. Schoenen, "On increasing the spectral efficiency more than $100 \%$ by user-inthe-control-loop," in Communications (APCC), 2010 16th Asia-Pacific Conference on, pp. 159-164, Oct 2010.

[55] A. Sang, X. Wang, M. Madihian, and R. Gitlin, "A load-aware handoff and cell-site selection scheme in multi-cell packet data systems," in Global Telecommunications Conference, 2004. GLOBECOM '04. IEEE, vol. 6, pp. 3931-3936 Vol.6, Nov 2004.

[56] M. Sanjabi, M. Razaviyayn, and Z.-Q. Luo, "Optimal joint base station assignment and downlink beamforming for heterogeneous networks," in Acoustics, Speech and Signal Processing (ICASSP), 2012 IEEE International Conference on, pp. 2821-2824, March 2012.

[57] ITU-R, "Guidelines for evaluation of radio interface technologies for IMTadvanced," Report M.2135, ITU-R, Nov. 2008.

[58] T. Beitelmal, R. Schoenen, and H. Yanikomeroglu, "On the impact of correlated shadowing on the performance of user-in-the-loop for mobility," in Communications (ICC), 2012 IEEE International Conference on, pp. 7040-7044, June 2012.

[59] S. S. Szyszkowicz and H. Yanikomeroglu, "A simple approximation to the interference from a large clustered wireless network with correlated shadowing," Wireless Communications, IEEE Transactions on, vol. 13, no. 8, pp. 4415-4423, Aug. 2014.

[60] M. Mirahsan, R. Schoenen, and H. Yanikomeroglu, "Statistical modeling of spatial traffic distribution with adjustable heterogeneity and BS-correlation in wireless cellular networks," in Proc. IEEE Globecom, (Austin, TX, USA), Dec. 2014.

[61] J. Møller and R. P. Waagepetersen, Statistical Inference and Simulation for Spatial Point Processes. CRC Press, 2004. 